Portland State University

PDXScholar

Summer 1-1-2012

\title{
Fundamental Properties of Functional Zinc Oxide Nanowires Obtained by Electrochemical Method and Their Device Applications
}

Athavan Nadarajah

Portland State University

Follow this and additional works at: https://pdxscholar.library.pdx.edu/open_access_etds

Part of the Optics Commons, and the Semiconductor and Optical Materials Commons Let us know how access to this document benefits you.

Recommended Citation

Nadarajah, Athavan, "Fundamental Properties of Functional Zinc Oxide Nanowires Obtained by Electrochemical Method and Their Device Applications" (2012). Dissertations and Theses. Paper 633. https://doi.org/10.15760/etd.633

This Dissertation is brought to you for free and open access. It has been accepted for inclusion in Dissertations and Theses by an authorized administrator of PDXScholar. Please contact us if we can make this document more accessible: pdxscholar@pdx.edu. 
Fundamental Properties of Functional Zinc Oxide Nanowires Obtained by Electrochemical Method and Their Device Applications

by

Nadarajah Athavan

A dissertation submitted in partial fulfillment of the requirements for the degree of

\author{
Doctor of Philosophy \\ in \\ Applied Physics
}

Dissertation Committee:

Rolf Könenkamp, Chair

Carl C. Wamser

Jun Jiao

Raj Solanki

Sergei Rouvimov

Portland State University

(C)2012 


\begin{abstract}
We report on the fundamental properties and device applications of semiconductor nanoparticles. $\mathrm{ZnO}$ nanowires and $\mathrm{CdSe}$ quantum dots were used, prepared, characterized, and assembled into novel light-emitting diodes and solar cells. $\mathrm{ZnO}$ nanowire films were grown electrochemically using aqueous soluble chloridebased electrolytes as precursors at temperatures below $90^{\circ} \mathrm{C}$. Dopants were added to the electrolyte in the form of chloride compounds, which are $\mathrm{AlCl}_{3}, \mathrm{CoCl}_{2}, \mathrm{CuCl}_{2}$, and $\mathrm{MnCl}_{2}$. The optical, magnetic, and structural properties of undoped and transitionmetal-ion doped $\mathrm{ZnO}$ nanowires were explored. Our results indicate that the as-grown nanowire structures have considerable internal strain, resulting in clearly visible lattice distortions in bright and dark-field transmission electron micrographs. Photo and electroluminescence studies indicate that the strain-induced defects strongly dominate any dopant-related effects. However, annealing at moderate temperature as well as laser annealing induces strain relaxation and leads to dopant activation. Hence, the optical and electrical properties of the nanowires significantly improve, allowing these nanowires to become feasible for use in the fabrication of solar cell and LED devices. In addition, the magnetic impurities incorporated into our $\mathrm{ZnO}$ nanowires show superparamagnetic behavior at room-temperature, while Al-doped and undoped $\mathrm{ZnO}$ nanowires show no magnetic behavior.
\end{abstract}

The electroluminescence (EL) is achieved from a vertical hybrid p-n junction LED arrangement consisting of a hole-conducting polymer and n-type $\mathrm{ZnO}$ nanowires, 
our group was the first to report this vertical nanowire-based LED in Könenkamp et al., 2004 [12]. The observed EL spectra show an ultraviolet excitonic emission peak and a broad defect-related emission band in the visible range. After annealing at $380^{\circ} \mathrm{C}$, the defect related EL peak exhibits a characteristic shift to higher wavelengths, where the magnitude of the shift is dependent on the dopant type. Aluminum incorporation exhibited the most improved exciton related-emission, leading to the emergence of a narrow excitonic luminescence peak around $390 \mathrm{~nm}$, which is close to the bandgap of ZnO. The comparison of spectra obtained from temperature-dependent photoluminescence (PL) measurements, before and after thermal annealing, also indicates that the optical activity of impurities changes noticeably upon annealing. The internal quantum efficiency for PL is measured to be as high as 16 percent for Al-doped samples annealed at $380^{\circ} \mathrm{C}$. The PL measurements also show that the excitonic luminescence is preferentially guided, while the defect related emission is more isotropically emitted.

The nanostructured heterojunction solar cell is designed such that thin CdSe quantum dot films are embedded between a $\mathrm{ZnO}$ nanowire film and a hole-conducting polymer layer. This arrangement allows for enhanced light absorption and an efficient collection of photogenerated carriers. Here, we present a detailed analysis of the pyridine solution and 1,2- ethanedithiol ligand exchange processes of the quantum dots, deposition processes of this quantum dot layer, the conformality of this layer on deeply nanostructured samples, and the effect of a surfactant-aided thermal annealing process. Annealing creates a structural conversion of the quantum dot layers into an extremely 
thin continuous poly-crystalline film, with typical grain diameters of 30-50 nm. This transition is accompanied by a loss of quantum confinement and a significant improvement of the charge transport in the CdSe layer. The combination of the solution and ligand exchange of CdSe quantum dots, as well as the deposition and optimized annealing processes of this quantum dot layer, resulted in solar cells with an opencircuit voltage up to $0.6 \mathrm{~V}$, a short circuit current of $\sim 15 \mathrm{~mA} / \mathrm{cm}^{2}$, an external quantum efficiency of 70 percent, and an energy conversion efficiency of 3.4 percent. This 3.4 percent efficiency is presently one of the best efficiencies obtained for this type of device. 


\section{Acknowledgements}

My Ph.D. program has come to an end with the help of unwavering guidance from my supervisor and my research group members, moral support from family members and friends, and all those who have served on my dissertation committee.

First and foremost, this research would not have been completed without the guidance of my doctoral advisor Professor Rolf Könenkamp and his continual financial support throughout the entirety of my graduate studies at Portland State University, by way of grants from the Office of Science, U.S. Department of Energy and the Oregon Nano and Microtechnologies Institute (ONAMI). He was very instrumental in defining the direction of this research, and was always available and open to discuss new ideas, to provide insightful proofreading of my draft papers and dissertation, and to answer my pertinent questions. From our research group, I am also indebted to Dr. Robert C. Word for his guidance in the design of our experiments, taking the time to teach me general chemistry, providing me with wonderful schematic diagrams of solar cells and LEDs, and for his willingness to discuss my results. I am appreciative of the time and effort that Dr. Tsutomu Shinagawa contributed to my solar cell research, in lending me instruction on various aspects of material synthesis and for our many useful discussions. Further gratitude goes to all my former and present research group members for their useful comments and suggestions regarding my research. 
Over the years, we have had various summer research students from local high schools via Saturday Academy (ASE), undergraduate students via Research Experience for Undergraduates (REU), and a high school physics teacher via the Partners in Science program who have been helpful and resourceful to my project. Among all these summer research participants, special thanks go to Mr. Thomas Smith, a physics teacher from Lake Oswego high school, for the enjoyable times we shared at the laboratory and for his help in the design and optimization of the solar cells that recently achieved an energy conversion efficiency of $3.4 \%$.

I am very grateful to Professor Carl C. Wamser for serving as the graduate coordinator of my thesis committee, for loaning me reference materials, and for allowing me to use his laboratory and attend his research group meetings. My profound gratitude also goes to Professor Sergei Rouvimov for his useful discussions, good ideas and directives on running HRTEM experiments, and making himself available to become a member of my thesis committee. Many special thanks go to Professor Jun Jiao and Professor Raj Solanki for participating as members of my thesis committee. I would also like to express my appreciation to the Portland State University Department of Physics for such a remarkable applied physics program.

I extend further gratitude to Dr. Björn Seipel for his insights, suggestions, and help with our XRD measurements. I also thank Mr. Benjamin Wutzke for his time and assistance in our XRD work. My apprection also goes to Dr. Erik Johansson for 
allowing me to use his centrifuge. Thank you to Mr. Chase Reinhart for his time and assistance with the operation of the centrifuge.

The temperature-dependent photoluminescence, vibrating sample magnetometer, and secondary ion mass spectroscopy measurements were performed at EMSL, a national scientific user facility sponsored by the Department of Energy's Office of Biological and Environmental Research located at Pacific Northwest National Laboratory. These measurements could not have been completed in time at EMSL without the help of Dr. Theva Thevuthasan, Dr. Zihua Zhu, Dr. Zheming Wang, and Dr. Timothy C. Droubay.

Special thanks also go to my friend Mr. Sean Burdeshaw who took on the great task of English proofreading my draft papers and dissertation.

Lastly, I would like to thank my family and friends for their encouragement and moral support during my studies. 


\section{Table of Contents}

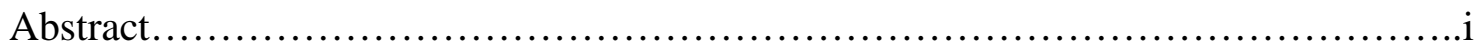

Acknowledgements..........................................................

List of Figures...........................................................

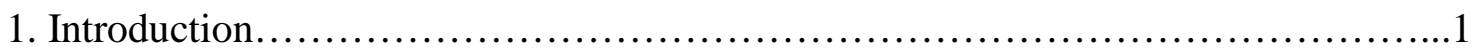

1.1 Overview..............................................................

1.2 Motivation.........................................................2

1.3 Developments in light-emitting diodes (LEDs) ...........................4

1.4 Developments in solar cells ........................................6

1.5 Physics backgroud................................................. 10

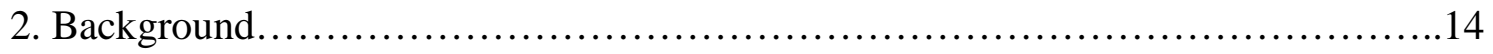

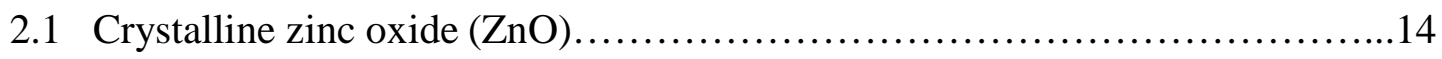

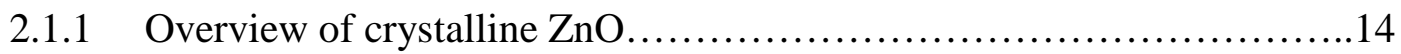

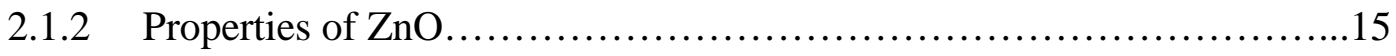

2.1.2.1 $\mathrm{ZnO}$ crystal structure......................................

2.1.2.2 $\mathrm{ZnO}$ electronic structure....................................16

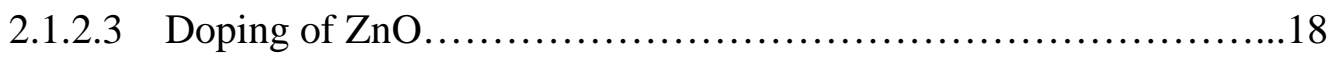

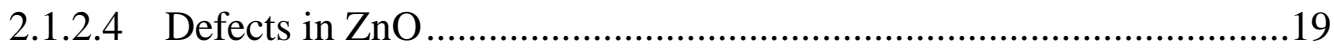

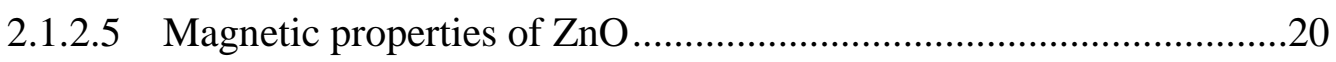

2.1.3 Overview of $\mathrm{ZnO}$ nanostructures................................22

2.1.4 Our research on $\mathrm{ZnO}$ material properties and growth.................24

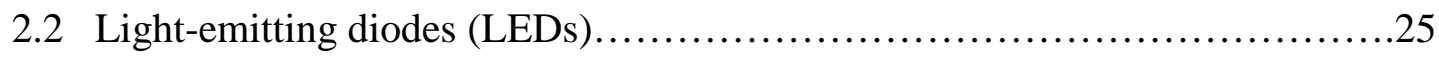

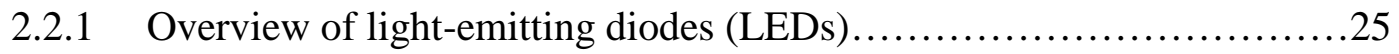


2.2.2 Development of III-nitride based light-emitting diodes (LEDs)..........26

2.2.3 Development of $\mathrm{ZnO}$ based light-emitting diodes (LEDs)..............26

2.2.3.1 Progress of $\mathrm{ZnO}$ thin film based LEDs.........................28

2.2.3.2 Progress of $\mathrm{ZnO}$ nanowire based LEDs........................30

2.2.4 Our vertical nanowire LED research................................ 31

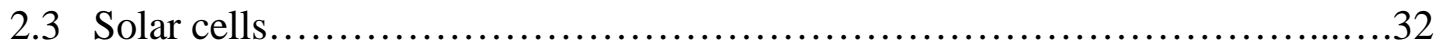

2.3.1 Overview of nanostructured solar cells............................ 33

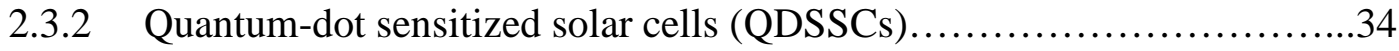

2.3.3 Exteremely thin absorber (ETA) solar cells...........................37

2.3.4 Inorganic-organic heterojunction solar cells......................... 39

2.3.5 Our solar cell research..........................................40

3. Experimental Methods...............................................43

3.1 Growth techniques of $\mathrm{ZnO}$ nanowires.................................. 43

3.1.1 Electrodeposition............................................44

3.1.1.1 Standard electrodeposition.................................45

3.1.1.2 Doping with transition metal ions..............................50

3.1.2 Chemical bath deposition.....................................51

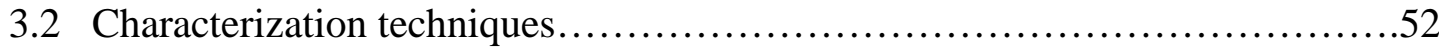

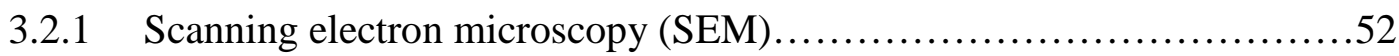

3.2.2 Transmission electron microscopy (TEM) .........................52

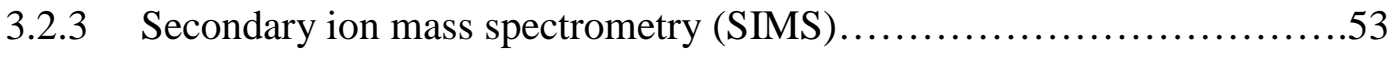

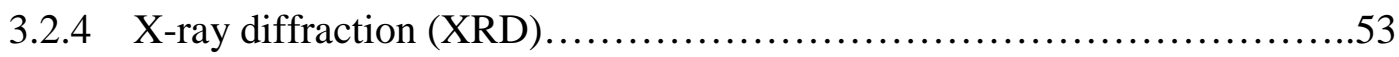

3.2.5 Vibrating sample magnetometer (VSM) .........................54

viii 
3.2.6 Photoluminescence and laser annealing............................55

3.3 $\mathrm{ZnO}$ nanowire/polymer based light-emitting diodes.......................56

3.3.1 Overview....................................................56

3.3.2 Polystyrene coating........................................... 57

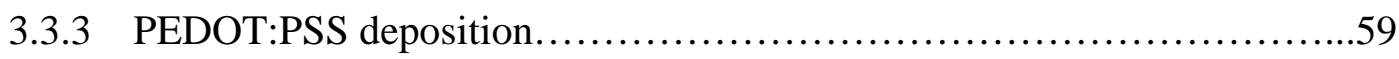

3.3.4 Deposition of gold contacts...................................60

3.3.5 Current-voltage characteristics................................61

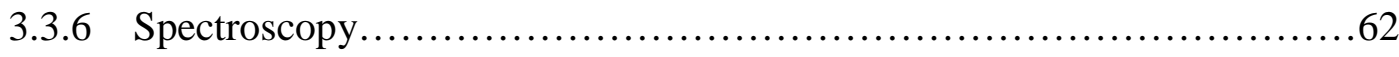

3.4 Nanowire-quantum-dot-polymer heterojunction solar cells.................64

3.4.1 ZnO thin film deposition.....................................64

3.4.2 Electrodeposition of $\mathrm{ZnO}$ nanowires.............................65

3.4.3 CdSe quantum dots.........................................66

3.4.3.1 Synthesis of CdSe qunatum-dots..............................................66

3.4.3.2 CdSe quantum dot deposition.............................67

3.4.3.2.1 Drop-coating of CdSe quantum-dots...................67

3.4.3.2.2 Layer-by layer dip-coating of CdSe quantum-dots..........68

3.4.3.3 $\mathrm{CdCl}_{2}$-aided thermal annealing of CdSe quantum dot film..........69

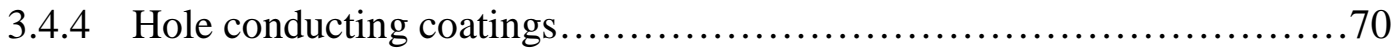

3.4.4.1 MEH-PVV coating...................................... 70

3.4.4.2 P3HT coating ............................................ 72

3.4.4.3 CuSCN deposition......................................73

3.4.5 Back contact deposition....................................... 74

3.4.6 Current-voltage measurements................................. 75 
3.4.7 Photoresponse measurements....................................76

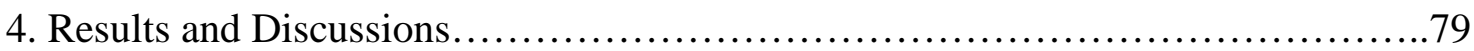

4.1 Electrochemical growth of $\mathrm{ZnO}$ nanowires and their fundamental

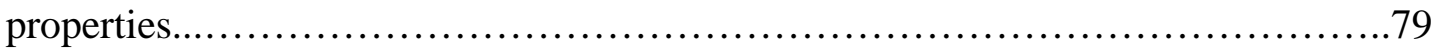

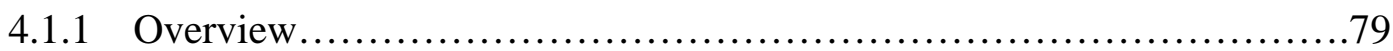

4.1.2 Morphology and structural properties of as-grown $\mathrm{ZnO}$ nanowires.........79

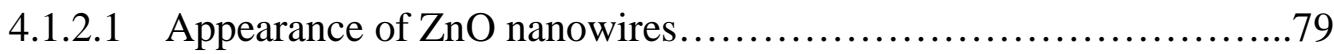

4.1.2.2 As-grown nanowires: crystal structure and defects.................81

4.1.3 Annealed $\mathrm{ZnO}$ nanowires: disappearance of strain defects at $\mathrm{T} \sim 380^{\circ} \mathrm{C} . .82$

4.1.4 Dopant incorporation: SIMS and TEM-EDX measurements..............84

4.1.5 Effects of doping: Magnetic properties.................................87

4.1.6 Effects of doping: Temperature-dependent photoluminescence

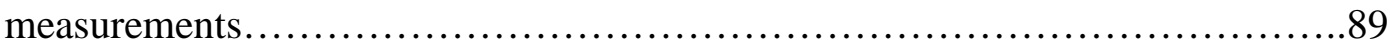

4.1.7 Laser Annealing: Room-temperature photoluminescence measurements..91

4.2 Electroluminescence from $\mathrm{ZnO}$ nanowires/polymer light-emitting diodes......95

4.2.1 Light-emitting diodes on planar substrates...........................95

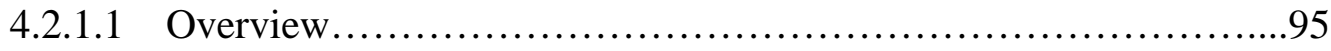

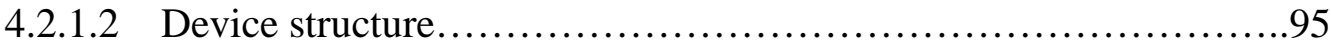

4.2.1.3 Basic results of our nanowire LED .............................96

4.2.1.4 Operational principles of our nanowire LED .....................97

4.2.1.5 Electroluminescence of as-grown and annealed $\mathrm{ZnO}$ nanowires......99

4.2.1.5.1 Electroluminescence of as-grown nanowires.................99

4.2.1.5.2 Electroluminescence of annealed nanowires.................101 
4.2.2 Light-emitting diodes on flexible substrates..........................104

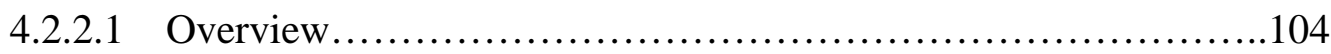

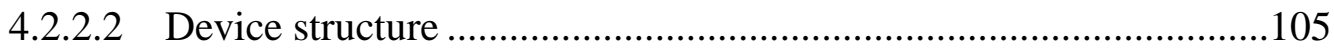

4.2.2.3 Morphology of $\mathrm{ZnO}$ nanowires on flexible substrates..............106

4.2.2.4 Current-voltage characteristics of as-grown and annealed $\mathrm{ZnO}$

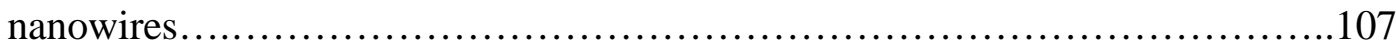

4.2.2.5 Electroluminescence of as-grown and annealed $\mathrm{ZnO}$ nanowires....108

4.3 Nanowire-quantum-dot solar cells and their performances...................111

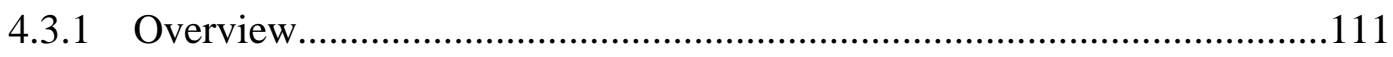

4.3.1.1 Why nanowires?......................................................................112

4.3.1.2 Why quantum dots?...................................................................112

4.3.1.3 Why a surface treatment for the quantum dot material?..................113

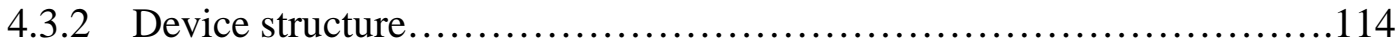

4.3.3 Concepts of extremely thin absorber solar cells......................115

4.3.4 Primary results of our solar cells....................................115

4.3.4.1 The un-annealed solar cell.................................... 115

4.3.4.2 Solar cell performances after two hour $\mathrm{CdCl}_{2}$-aided thermal annealing

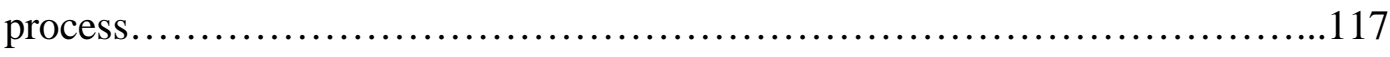

4.3.4.3 Effect of extended $\mathrm{CdCl}_{2}$-aided thermal annealed solar cells........118

4.3.5 Operational principles of our solar cell..............................119

4.3.6 Properties of CdSe quantum dot layer.................................121

4.3.7 Crystal structure of CdSe quantum-dot film .........................123 
4.3.8 Effects of various back contact layers and the formation of $\mathrm{ZnO}$ thin film prior to electrodeposition of $\mathrm{ZnO}$ nanowires............................. 126

4.3.8.1 Electrical measurements...................................126

4.3.8.2 Comparative photoresponse measurements....................128

4.3.9 Chemical treatment of the quantum dot with ligand and solvent

materials............................................................. 131

4.3.9.1 Overview..............................................131

4.3.9.2 Appearance of 1,2-ethanedithiol treated CdSe quantum-dot film...132

4.3.9.3 Photovoltaic properties of solar cells made with 1,2-ethanedithiol

treated CdSe quantum-dots......................................... 135

4.3.9.4 Photoresponse measurements: The effect of pyridine solution

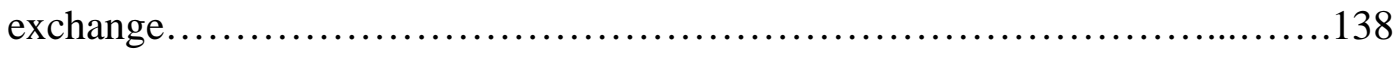

4.3.9.5 Electrical measurements: Improved transport with pyridine and 1,2ethanedithiol treatment in the preparation of nanowire-quantum-dot solar cells..139

4.3.9.6 The effect of ageing on photovoltaic performance................141

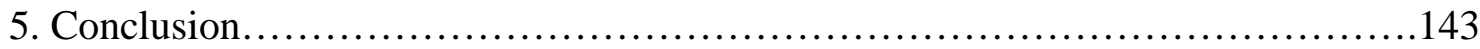

References......................................................... 147

xii 


\section{List of Figures}

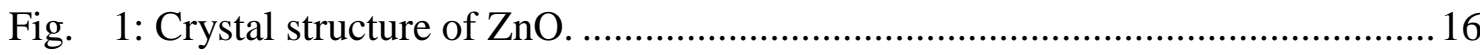

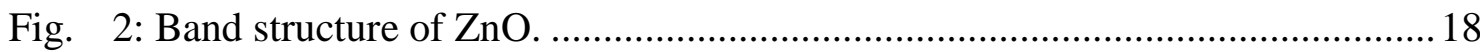

Fig. 3: Various forms of $\mathrm{ZnO}$ nanostructures: nanowires, nanobelts, nanosprings,

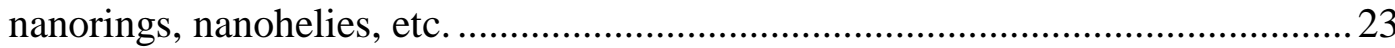

Fig. 4: SEM micrograph of transparent, fluorinated $\mathrm{SnO}_{2}$ /glass substrate................46

Fig. 5: Schematic diagram of electrodeposition setup........................................ 47

Fig. 6: SEM micrographs of (a) nanowires and (b) a single nanowire embedded in polystyrene (PS) solution of two layers of $50 \mathrm{~g} / \mathrm{L}$ HMW. .58

Fig. 7: SEM micrograph of very thin polystyrene coating on $\mathrm{ZnO}$ nanowire film. ....59

Fig. 8: SEM micrograph of very thick polystyrene coating on $\mathrm{ZnO}$ nanowire film....59

Fig. 9: SEM micrograph of PEDOT:PSS coated PS/ZnO nanostructures..................60

Fig. 10: Schematic diagrams of $\mathrm{ZnO}$ nanowire-based LED arrangements on (a) planar and (b) flexible substrates. 61

Fig. 11: Schematic diagram of electrical and optical measurements setup ................63

Fig. 12: SEM micrograph of spray-deposited $\mathrm{ZnO}$ thin film covered FTO substrate. 65

Fig. 13: Digital photograph of various sizes of CdSe qunatum dots. ........................67

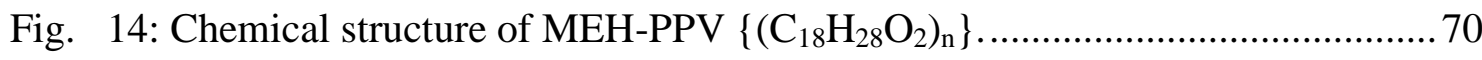

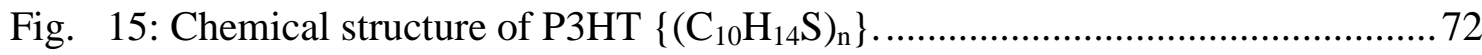

Fig. 16: Schematic diagram of nanowire-qunatum-dot solar cell............................ 74

Fig. 17: Schematic diagrams of (a) IV and (b) photoresponse measurement setups. 
Fig. 18: SEM micrographs of free standing (a) $5 \mu \mathrm{M}$ Al doped and (b) $20 \mu \mathrm{M}$ Al doped $\mathrm{ZnO}$ nanowires on an FTO substrate.

Fig. 19: (a) HRTEM image and (b) selected area electron diffraction pattern of an asgrown $\mathrm{ZnO}$ nanowire 81

Fig. 20: Dark-field TEM micrographs of a $\mathrm{ZnO}$ nanowire in the as-grown state........ 82

Fig. 21: (a) HRTEM and (b) Dark-field TEM micrographs of a $\mathrm{ZnO}$ nanowire in the annealed state at $380^{\circ} \mathrm{C}$ (the dislocations are marked with white color rectangular boxes). 83

Fig. 22: $\mathrm{SAD}$ patterns for a $\mathrm{ZnO}$ nanowire in the (a) as-grown, and (b) annealed state $380^{\circ} \mathrm{C}$ (the diffraction spot size is reduced in the annealed state). .84

Fig. 23: (a) The STEM-EDX line profile and (b) SIMS sputtering depth profile of a $5 \mu \mathrm{M} \mathrm{Al}$ doped $\mathrm{ZnO}$ nanowire annealed at $380^{\circ} \mathrm{C}$. .85

Fig. 24: The SIMS count ratios of the doped $\mathrm{ZnO}$ nanowires as a function of precursor concentrations in the solution. 86

Fig. 25: The room-temperature magnetization curves of (a) various magnetic impurity (such as $\mathrm{Cu}, \mathrm{Co}$, and $\mathrm{Mn}$ ) doped $\mathrm{ZnO}$ nanowires, (b) $\mathrm{Al}$ doped $\mathrm{ZnO}$ nanowires, and (c) undoped $\mathrm{ZnO}$ nanowires .88

Fig. 26: Room-temperature photoluminescence spectra of Al-doped $\mathrm{ZnO}$ nanowire samples before and after annealing 89

Fig. 27: Integrated intensity of a near band-edge emission at various temperatures for the undoped and Al-doped $\mathrm{ZnO}$ nanowire samples 91

Fig. 28: Improvement of the excitonic luminescence in $\mathrm{ZnO}$ nanowires due to laser annealing with $10 \mathrm{~mJ}$ pulses incident directly onto the nanowires .92 xiv 
Fig. 29: SEM micrograph showing the melted $\mathrm{ZnO}$ nanowires on $\mathrm{SnO}_{2}$-coated glass substrate after $30 \mathrm{~min}$ exposure to $10 \mathrm{~mJ}$ laser pulses. .93

Fig. 30: Near band-edge photoluminescence at $390 \mathrm{~nm}$ as a function of laser anneal times at various laser pulse energies for (a) direct illumination on $\mathrm{ZnO} / \mathrm{FTO}$ and (b) illumination through various substrates. .93

Fig. 31: Near-bandgap and sub-bandgap emission peaks as a function of detection angles. 94

Fig. 32: Schematic diagram of a $\mathrm{ZnO}$ nanowire-based LED arrangement on a planar FTO/glass substrate. 96

Fig. 33: (a) Current-voltage characteristics and (b) electroluminescence spectrum of undoped $\mathrm{ZnO}$ nanowire-based LED. 97

Fig. 34: Band energy levels of the nanowire/polymer LED device arrangement ........98

Fig. 35: Electroluminescence spectra of various transition-metal-ion doped as-grown $\mathrm{ZnO}$ nanowire LED devices 100

Fig. 36: PL and EL spectra obtained from as-grown $\mathrm{ZnO}$ nanowires. 101

Fig. 37: Electroluminescence spectrum of various transition-metal-ion doped $380^{\circ} \mathrm{C}$ annealed $\mathrm{ZnO}$ nanowire LED devices, which demonstrate that the visible luminescence is dominated by metal impurities 102

Fig. 38: PL and EL spectra obtained from $\mathrm{Al}$ doped $\mathrm{ZnO}$ nanowires annealed at $380^{\circ}$ C. The PL is obtained with excitation of a He-Cd laser at $325 \mathrm{~nm}$, and the EL is obtained with excitation voltage of $10 \mathrm{~V}$, at room temperature in air. 104

Fig. 39: Schematic diagram of a $\mathrm{ZnO}$ nanowire-based LED arrangement on a flexible substrate. 105 
Fig. 40: The SEM image of $\mathrm{ZnO}$ nanowires grown in electrodeposition on a flexible transparent ITO/PET substrate 106

Fig. 41: $\mathrm{ZnO}$ nanowires spin-coated with insulating layer from a polystyrene/toluene solution. 107

Fig. 42: The electrical characteristics of Various LED structures. 108

Fig. 43: Electroluminescence spectra of flexible LEDs as compared to a planar LED. 110

Fig. 44: Schematic layout of our nanowire-quantum-dot solar cell 114

Fig. 45: (a) Spectral response and (b) light and dark current-voltage characteristic plots of our un-annealed nanowire-quantum-dot solar cell. 116

Fig. 46: (a) Current-voltage plot and (b) photoresponse behavior as a function of wavelength for a nanowire-quantum-dot solar cell annealed at $380^{\circ} \mathrm{C}$ in the presence of $\mathrm{CdCl}_{2} /$ air for two hours 118

Fig. 47: Improvement of solar cell performance by increasing anneal times for annealing at $380^{\circ} \mathrm{C}$ in $\mathrm{CdCl}_{2} /$ air 119

Fig. 48: Schematic band diagram of a nanowire-quantum-dot-polymer heterojunction solar cell 120

Fig. 49: SEM micrographs showing the CdSe quantum dot film-covered $\mathrm{ZnO}$ nanowires in (a) an as-grown state, and after annealing in $\mathrm{CdCl}_{2}$ for (b) two hours and (c) 24 hours 122

Fig. 50: a) HRTEM micrograph of the $\mathrm{CdSe} / \mathrm{ZnO}$ interface of an as-deposited quantum dot film on a $\mathrm{ZnO}$ nanowire. The image shows that individual quantum dots are single-crystalline. b) The $\mathrm{CdSe} / \mathrm{ZnO}$ interface of a nanowire from the same xvi 
sample but annealed for $24 \mathrm{~h}$ at $380^{\circ} \mathrm{C}$. Due to annealing, the CdSe is now polycrystalline. c) TEM-EDX line profile of un-annealed $\mathrm{CdSe} / \mathrm{ZnO}$ nanostructures. d) TEM-EDX line scans across the CdSe-coated $\mathrm{ZnO}$ nanowires after $24 \mathrm{~h}$ anneal at $380^{\circ}$ C. e) TEM-EDX line scans across the CdS-coated $\mathrm{ZnO}$ nanowires after $2 \mathrm{~h}$ anneal at $380^{\circ} \mathrm{C}$ 125

Fig. 51: Dark J-V curves of $\mathrm{ZnO} / \mathrm{CdSe}$ solar cell structure prepared with different $\mathrm{p}$ layer coating: (a) $\mathrm{ZnO} / \mathrm{CdSe} / \mathrm{MEH}-\mathrm{PPV}, \quad$ (b) $\mathrm{ZnO} / \mathrm{CdSe} / \mathrm{P} 3 \mathrm{HT}$, (c) $\mathrm{ZnO} / \mathrm{CdSe} / \mathrm{CuSCN}$, and (d) $\mathrm{ZnO}$ thin film/ZnO/CdSe/P3HT 127

Fig. 52: Light $\mathrm{J}-\mathrm{V}$ curves of $\mathrm{ZnO} / \mathrm{CdSe}$ solar cell structure prepared with different $\mathrm{p}$ layer coating: (a) $\mathrm{ZnO} / \mathrm{CdSe} / \mathrm{MEH}-\mathrm{PPV}, \quad$ (b) $\mathrm{ZnO} / \mathrm{CdSe} / \mathrm{P} 3 \mathrm{HT}, \quad$ (c) $\mathrm{ZnO} / \mathrm{CdSe} / \mathrm{CuSCN}$, and (d) $\mathrm{ZnO}$ thin film/ZnO/CdSe/P3HT 128

Fig. 53: External quantum efficiency as a function of wavelength for $\mathrm{ZnO} / \mathrm{CdSe}$ nanostructured solar cells prepared with different p-layer coating: $\mathrm{ZnO} / \mathrm{CdSe} / \mathrm{MEH}-\mathrm{PPV}$, (b) $\mathrm{ZnO} / \mathrm{CdSe} / \mathrm{P} 3 \mathrm{HT}$, (c) $\mathrm{ZnO} / \mathrm{CdSe} / \mathrm{CuSCN}$, and (d) $\mathrm{ZnO}$ thin film/ZnO/CdSe/P3HT 130

Fig. 54: SEM micrographs showing (a) the $\mathrm{ZnO}$ nanowire film covered with octadecylamine capped CdSe quantum dot film prior to thermal annealing, showing the polymeric nature, (b) the $\mathrm{ZnO}$ nanowire film coated with EDT treated CdSe prior to thermal annealing, and (c) the $\mathrm{ZnO}$ nanowire film coated with EDT treated CdSe after $2 \mathrm{~h}$ annealing 134

Fig. 55: External quantum efficiency as a function of wavelength for un-annealed $\mathrm{ZnO}$ thin film/ZnO/CdSe/P3HT nanostructured solar cells. 136 
Fig. 56: External quantum efficiency as a function of wavelength for $380^{\circ} \mathrm{C}$ annealed nanowire-quantum-dot solar cells

Fig. 57: External quantum efficiency as a function of wavelength for 380o C annealed nanowire-quantum-dot solar cells prepared with CdSe quantum dots that have undergone pyridine solution and EDT ligand exchange (shown by red curve) and CdSe quantum dots that are suspended in Toluene and caped with EDT ligand molecules (shown by black curve) 139

Fig. 58: Current-voltage characteristics under dark and $85 \mathrm{~mW} / \mathrm{cm}^{2}$ illumination conditions for a nanowire-quantum-dot solar cell prepared with CdSe quantumdots that have undergone pyridine solution and EDT ligand exchange. 140

Fig. 59: External quantum efficiency plots as a function of wavelength for a solar cell that has been kept in ambient air conditions at room-temperature for various periods of time 142 


\section{Introduction}

\subsection{Overview}

Beginning with a general introduction to the concepts of sustainable energy production and conversion, this chapter provides an overview of the motivations for my thesis, detailing the progress in light-emitting diode (LED) and solar cell technologies, with a special emphasis on our nanowire/polymer LEDs and nanowire-quantum-dotpolymer heterojunction solar cells, as well as the physics underlying these devices. The first section of this chapter addresses the importance of developing sustainable and renewable energy production and conversion technologies, with a focus on solutionprocessed nanoscale semiconductor materials and the potential that they offer for creating simple and low cost devices. The second section outlines developments in LED technologies with a primary interest in the use of $\mathrm{ZnO}$ based LEDs for future lighting applications. In the third section, we focus on the evolution of photovoltaic technologies, with a particular focus on third generation solar cells as a means to inexpensive energy conversion. The final section concentrates on the physics underlying LED and solar cell operation, using the generalized Planck's law. 


\subsection{Motivation}

Oil, gas, and coal are the primary sources of energy sustaining our present global energy demands. However, the burning of these fossil fuels not only produces energy but also releases carbon dioxide and other adverse compounds into the Earth's atmosphere, ultimately resulting in climate change. The harvesting, processing, storage, and distribution of these fossil fuels also have major negative impacts on the environment, significantly affecting both human health and the ecosystem. By 2020 , the global demand for energy will have grown to $649 \times 10^{18} \mathrm{~J}(612$ quadrillion British thermal units (Btu)), leading to an increase in carbon emissions, and further aggravating the already existing problems related to the green house effect [1]. Therefore, the search for every suitable technology that could assist in solving this issue in a sustainable way, and lead to more economic growth, is a present necessity. In this thesis, we address this broad agenda through two main areas of research: (1) Exploring novel light emitting diodes for lighting and display applications and (2) developing new solar cells for energy conversion. Both topics strongly focus on the potential of improving cost and efficiency in these two technologies.

Light-emitting diodes (LEDs) have received significant research interest due to their energy saving capabilities. In terms of their energy efficiency, lifetime, and lowcost manufacturability, LEDs are now superior to conventional illumination sources, such as incandescent and fluorescent lamps. Presently, conventional lighting technologies such as fluorescent lamps provide only a 13\% external quantum efficiency 
[1] whereas the efficiency of LEDs is now at 30\% [2], and could reach values as high as $50 \%$ within the near future [1]. A large number of lighting systems, including traffic lights, signs, and displays have already replaced the traditional light bulbs with LEDs. LEDs are semiconductor devices, and the emitted color is determined by the bandgap of the materials. Therefore, LEDs are inherently monochromatic emitters, and applications with color requirements are very efficient. The creation of white light from inherently monochromatic emitters can be achieved using dichromatic, trichromatic, or tetrachromatic techniques.

From an energy point of view, the use of solar cells, in which energy is harvested directly from sunlight, is sufficient to fulfill the needs of today's global energy consumption. However, current cost levels of solar cells have so far prevented their wide spread terrestrial use. As of 2008 photovoltaics contribute more than $5 \mathrm{GW}$ [3] to the world's total electricity production of $13.5 \mathrm{TW}$ [4]. Over the past 10 years solar cells have expanded dramatically in residential and commercial use, with some countries committing large amounts of funding to continue this expansion. Forecasts predict that PV based power production will reach above 20 GW by 2015 [3].

Technical developments in both light-emitting diodes and photovoltaic cells share many of the same challenges, such as achieving high efficiencies, obtaining long lifetimes, and maintaining low manufacturing costs. Traditionally, LED and PV technologies have been composed of crystalline and polycrystalline thin film semiconductors, but more recently researchers have identified solution-processed 
nanostructured materials as a promising route towards the creation of cost-efficient large-scale solid state lighting and solar photovoltaics. By exploring new processes and architectures for solution-grown LEDs and solar cells, we hope to address the obvious challenges to cost efficiency, reliability and durability.

\subsection{Developments in light-emitting diodes (LEDs)}

In 1907, H. J. Round and his colleagues reported the first observation of electroluminescence from a SiC crystal $[5,6]$. This device exhibited rectifying currentvoltage characteristics with operating voltages in the range of $10 \mathrm{~V}-110 \mathrm{~V}$. Having first been reported by $\mathrm{H}$. Welker in 1952, the invention of the III-V compound semiconductor opened the door for a new age of light emitter technologies [7]. Nichia Chemical Industries Corporation researchers Shuji Nakamura and Takashi Mukai have made numerous contributions to the development of GaN growth, LEDs, and lasers. Their work includes the discovery of and the demonstration of the first pulsed and continuous wave $\mathrm{GaInN} / \mathrm{GaN}$ current injection blue laser operating at room temperature $[8,9]$. Since then, group III nitride materials such as AlN, GaN and $\mathrm{InN}$ have been predominantly used in the construction of LEDs.

Recently, however, those working in the area of optoelectronic research have recognized $\mathrm{ZnO}$ as a promising semiconductor material for the fabrication of ultraviolet light emitting diodes and lasers. $\mathrm{ZnO}$ and $\mathrm{GaN}$ share many similar physical properties. Both have wurtzite crystal structures, almost the same in-plane lattice parameter (lattice 
mismatch of $1.8 \%$ ), and a wide band gap at room-temperature. However, $\mathrm{ZnO}$ exhibits some properties that are more favorable than those of $\mathrm{GaN}$, including having a larger exciton binding energy of $60 \mathrm{meV}$, a higher radiation hardness, the availability of large area growth, and the ability to be solution processed, thus making it a relatively low cost material. Moreover, $\mathrm{ZnO}$ is a versatile and functional material, and can be grown into various nanostructured morphologies including nanowires, nanorods, nanorings, and nanotubes. These nanostructures provide a high crystallinity and an enhanced surface-to-volume ratio when compared to $\mathrm{ZnO}$ polycrystalline thin films. Due to a bandgap of $3.4 \mathrm{eV}, \mathrm{ZnO}$ nanostructures are attractive for blue and ultraviolet optoelectronic and transparent electronic applications. Presently, however, there is still a lack of high quality p-type $\mathrm{ZnO}$. This deficiency continues to prevent the fabrication of $\mathrm{ZnO}$ homojunction devices. The only alternative to $\mathrm{p}-\mathrm{n}$ homojunctions is to obtain functional heterojunction devices through the combination of $\mathrm{ZnO}$ with other p-type organic or inorganic semiconductor materials. Recent heterojunction LEDs have been constructed by combining $\mathrm{n}-\mathrm{ZnO}$ with various p-type semiconductor layers such as $\mathrm{Si}$ [10] and GaN [11].

In our LED research, we were among the very first to report promising device properties of nanostructured n-type $\mathrm{ZnO}$ nanowires combined with p-type polymer films in hybrid p-n heterojunction LEDs $[12,13]$. A broad-band electroluminescence across the spectral region from $350 \mathrm{~nm}$ to $850 \mathrm{~nm}$ was observed from the as-grown heterojunction LED at forward bias voltages. Further thermal and chemical processing improved the sample quality. We were able to demonstrate that this processing removes 
lattice strain, converting the strain into local defects, and that this conversion can lead to a significant increase in electroluminescence intensity and a shift of the emission peak towards the ultraviolet region. Our best samples exhibited a narrow UV electroluminescence spectrum centered at $393 \mathrm{~nm}$. Through the incorporation of metal ions into the nanowires during growth, the electroluminescence spectra can be tuned to any color in the visible region, even approaching near-infrared [14]. We were also able to use the same LED architecture for the fabrication of flexible LEDs by simply replacing the glass substrate with a polyethylene terephthalate (PET) film, attaining similar electroluminescence results as compared to results that were previously obtained from planar LED devices [15]. These flexible LEDs have the potential to be used in low-level light applications, such as displays.

\subsection{Developments in solar cells}

In 1839, Edmund Bequerel reported the first effect of photovoltaic activity when he observed an electric current while shining light onto a silver coated platinum electrode immersed in an electrolyte [16]. Four decades after the discovery of photovoltaic action, the first solid state photovoltaic cell was constructed by Charles Fritts in 1883 [17]. His device consisted of a layer of selenium sandwiched between gold and another metal contact and exhibited only around 1\% energy conversion efficiency. This early solar cell made use of a Schottky junction, in which a semitransparent metal layer deposited on the semiconductor provides an asymmetric electronic junction for photovoltaic action. In 1954, the first crystalline silicon ( $\mathrm{Si}$ ) solar 
cell was constructed by Chapin and his colleagues at Bell laboratories [18]. This first silicon solar cell exhibited an energy conversion efficiency of $6 \%$, which was six times higher than the best previous device. This type of solar cell opened the door to what is now called a first generation photovoltaic device. The first generation Si based singlejunction devices have an efficiency limit of $31 \%$ under 1 sun illumination as shown by Shockley and Queisser [19]. Many of these early Si based solar cells were primarily developed for applications in aeronautical industries. Strong efforts towards the improvement of these crystalline Si cells, which have led to today's best cells with an efficiency of $25 \%$ [20]. However, the problems associated with the high cost of Si technology were apparent from the beginning, prompting researches to recognize that low-cost solar cells would only be produced if cost-efficient raw materials as well as inexpensive material synthesis and processing technologies were utilized.

A second generation of photovoltaic devices based on thin-film technologies began in the 1980s. This type of solar cell offers a large reduction in material costs due to the elimination of wafer technology in favor of thin film processes applied to materials such as microcrystalline and amorphous silicon, and direct band gap materials like cadmium telluride (CdTe), copper indium gallium (di)selenide (CIGS), and copper indium (di)selenide (CIS). As single junction cells, these solar cells still cannot obtain efficiencies higher than those given by the Shockley-Queisser limit.

Most recent developments in photovoltaics are implemented in third generation solar cells, which combine novel materials such as nanowires, quantum dots, dyes, and 
conjugated polymer networks with advanced architectures and novel operational principles. These nanostructured materials provide the potential for developing a lowcost and high-efficient photovoltaic technology that is economically viable for largescale power generation using environmentally safe materials with virtually unlimited availability. A number of third generation cells can be grown on flexible substrates by means of well established printing techniques. The capability of using inexpensive plastic substrates in a simple and large scale printing process, without a need for vacuum, can reduce the manufacturing cost substantially, and will result in a much shorter energetic pay-back time than that obtained by more conventional technologies. Some of the nanostructured solar cells also have theoretical efficiencies that go beyond the Shockley and Queisser limit of 31\%. However, the ultimate goal is to create solar cells with efficiencies that can reach to the Carnot efficiency limit of $66 \%$ for 1 Sun irradiation. These highly efficient devices will require multiple bandgap absorbers as well as a much refined architecture. Polymer based solar cells with 10 different bandgaps and reaching efficiencies up to $8 \%$ have recently been demonstrated [21].

A major breakthrough for nanostructured solar cells came in 1991 when O'Regan and Grätzel reported an efficiency of $7 \%$ in a cell that was created using a nanoporous wide bandgap semiconductor sensitized by tightly absorbed dye molecules [22]. This demonstration strongly motivated the further use of nanostructures in photovoltaics. Nanostructures are now being implemented into solar cells for various device configurations including nanoporous dye-sensitized cells, inorganic nanowire 
cells, quantum dot cells, organic/inorganic hybrid cells, organic cells, and extremely thin absorber (ETA) cells.

In our solar cell research, we demonstrate nanostructured solar cells composed of novel solution-processed nanoparticles, such as $\mathrm{ZnO}$ nanowires and $\mathrm{CdSe}$ quantum dots, as their main components. These ingredients will allow for large-scale solar photovoltaic fabrication with low manufacturing costs [23]. A detailed analysis of CdSe quantum dot layer deposition processes led us to implement the use of pyridine solution and EDT ligand exchange for the replacement of the initial solution and ligand of the as-synthesized quantum dot precursor material, yielding a highly conformal coating on deeply nanostructured samples and excellent current transfer across the interface. We also studied, in detail, the effects of a surfactant-aided thermal anneal process, and were able to show that the annealing process causes a structural conversion of the quantum dot layers into a very thin continuous polycrystalline film with grain diameters of 30-50 nm. This transition is accompanied by both a loss of quantum confinement and a significant improvement of the charge transport in the layer. Through the optimization of our deposition and processing methods, we were able to report solar cells with an open-circuit voltage of up to $0.6 \mathrm{~V}$ and a short circuit current of $\sim 15 \mathrm{~mA} / \mathrm{cm}^{2}$ under 85 $\mathrm{mW} / \mathrm{cm}^{2}$ illumination. Our best current energy conversion efficiency for this type of extremely-thin absorber solar cell is $3.4 \%$. This efficiency is among the very best for this type of solar cell. 


\subsection{Physics background}

The physics underlying the function of LEDs and solar cells are related, as both devices depend upon the interaction of photons and electrons. LEDs convert electrical current into photons, whereas solar cells convert photons into electrical power. In LEDs, excess electrons and holes are injected into a semiconductor via selective contacts. The injected electrons and holes recombine in the center of the device, thereby producing radiation, atomic vibrations, and heat. However, in an ideal LED, the only active process to occur would be that of radiative recombination, and other competing processes would not take place. Radiative recombination occurs when an electron in the conduction band recombines with a hole in the valence band, leading to the emission of a photon. Essentially, the energy lost by the electron is $E_{g}$ and appears as the photon energy $h v$, which is shown by the following equation.

$$
h v=E_{g}
$$

The Van Roosbroeck-Shockely method is the classic approach used to calculate the spontaneous radiative recombination rate under detailed balance conditions [24]. This technique requires the knowledge of only a few basic parameters, the bandgap energy, the absorption coefficient, and the refractive index. However, this model does not take into account non-equilibrium conditions, i.e. external radiative excitation or an applied voltage. A generalized Planck's law, introduced by Würfel, allows one to calculate the photon emission rate for both direct and indirect transitions under non- 
equilibrium conditions [25]. The electron and hole energies are calculated statistically using a non-zero chemical potential. This chemical potential, in the case of LEDs, is essentially just the voltage applied to the injecting contacts.

The LED emission intensity at an applied voltage $\mathrm{V}$ is then given by the following equation:

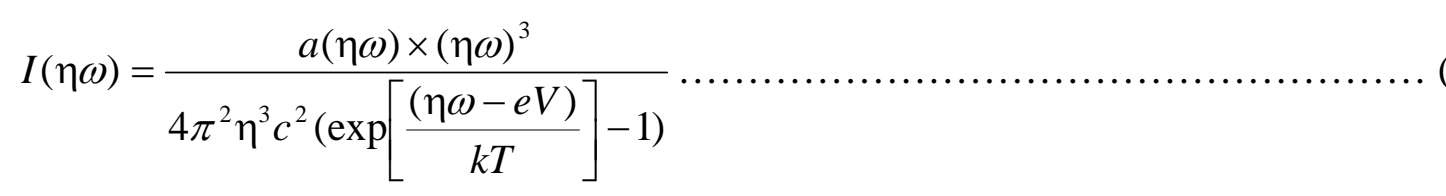

In this equation, $V$ is the applied voltage, which mainly affects the intensity of the emission spectrum; $T$ is the temperature of the LED, affecting the intensity and the shape of the spectrum; $a(\eta \omega)$ is the absorptivity of the material. In order to maximize the radiative recombination process, all other processes, including non-radiative recombinations and coupling losses, are to be avoided.

In solar cells, electron-hole pairs are generated by the absorption of photons in the semiconductor material. The complementary physical process to the absorption of photons is radiative recombination, i.e. the same process that is maximized in LEDs. In order to maximize device efficiency in solar cells, it is necessary to minimize all the fundamental losses. These fundamental losses can be divided into two distinct categories: extrinsic losses and intrinsic losses. Extrinsic losses, such as reflection losses, resistive losses, and non-radiative recombination losses can substantially limit 
device efficiency, but are avoidable. Intrinsic losses, such as absorption losses, thermalization losses, and radiative recombination losses are considered to be unavoidable losses in the solar cell design. The process of radiative recombination is an unavoidable loss process in solar cells, as it is required by the generalized Planck law. Two other substantial and unavoidable loss processes are, absorption losses for photon energies below the bandgap value and, the thermalization of hot carriers. The mechanisms of all these loss processes are easily understood and can be calculated using well-known formulas. The radiation loss can again be calculated from the generalized Planck law.

The radiation loss is then:

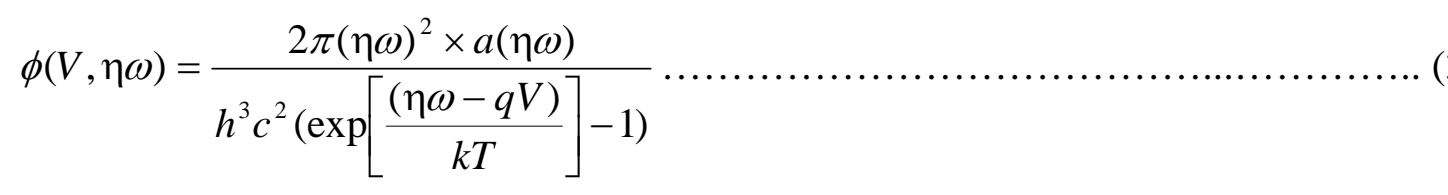

As previously defined, the term $q V$ is the chemical potential of electron-hole pairs $\left(\mu_{e h}\right)$, as well as a voltage of the solar cell in an ideal case, where the absorption of a photon results in the creation of a single electron-hole pair, thus causing the number of absorbed photons to be equal to the number of excited electron-hole pairs. This radiation process is linked to the generation of electrical output power, as $\mathrm{V}$ is the voltage of the solar cell. The generalized Planck law, in which the photon flux and spectral distribution are coupled to the device voltage, thus becomes a fundamental theoretical concept for understanding both light generation and energy conversion. 
Today, most solar cells, and many LEDs, are still much less efficient than they should be on the basis of these fundamental considerations. From a practical development point of view, researchers often start out with very small efficiencies in their first devices, frequently in the $0.1 \%$ range. Most of their work then focuses on a reduction of avoidable losses in order to show the basic feasibility of a new device idea. Once this feasibility is demonstrated, their next step is to improve the design of the device in order to obtain a device performance that is commercially viable. The possibility of commercialization is now real, causing aspects of the device relating to cost, reliability, durability, and many other practical issues to become immediately important. The work in this thesis involves the very early steps in this sequence: demonstrating the feasibility of new device architectures, the fabrication of a working model device, and finding methods for the improvement of this working device. 


\section{Background}

\subsection{Crystalline zinc oxide $(\mathrm{ZnO})$}

\subsubsection{Overview of crystalline $\mathrm{ZnO}$}

Zinc oxide $(\mathrm{ZnO})$ has become an important material for many applications in electronics, optics, and photonics because of its direct wide-bandgap of $3.4 \mathrm{eV}$, its ntype conductivity, and its large exciton binding energy of $60 \mathrm{meV}[11,12,26,27,28]$. $\mathrm{ZnO}$ is transparent to visible light and can be made highly conductive by introducing substitutional doping impurities [29]. Its electrical properties can be changed from an insulator to an n-type semiconductor to a metal by controlling the doping level while largely keeping the optical transparency. These characteristics make $\mathrm{ZnO}$ useful for transparent electrodes in flat-panel displays and solar cells. Yet, p-doping of $\mathrm{ZnO}$ has remained a challenge due to the self-compensation of intrinsic donors, such as oxygen vacancies and zinc interstitials, as well as the low solubility of extrinsic dopants. Growth of p-type $\mathrm{ZnO}$ crystals is one of the important issues that need to be overcome before $\mathrm{ZnO}$ could potentially make inroads into the world of optoelectronics devices. There has been some progress made with achieving p-type conductivity in $\mathrm{ZnO}$ films using various growth methods and group V dopant elements (N, P, As, and Sb) [30-36]. However, a reliable and reproducible high quality p-type conductivity has not been achieved for $\mathrm{ZnO}$ yet. As a result, it remains the most critical topic in $\mathrm{ZnO}$ research today, and many researchers continue to direct their efforts at solving this problem. At 
the same time, most of the research efforts are directed to create $\mathrm{p}-\mathrm{n} \mathrm{ZnO}$ homojunctions for practical device applications [37, 38].

\subsubsection{Properties of $\mathrm{ZnO}$}

\subsubsection{1 $\mathrm{ZnO}$ crystal structure}

$\mathrm{ZnO}$ is a binary chemical compound from group the II-IV group of elements in the periodic table. The crystal structures of $\mathrm{ZnO}$ are wurtzite, zinc blende, and rocksalt. Among all of these, the wurtzite structure is thermodynamically more stable at ambient conditions where each anion is bonded to four cations at the corners of a tetrahedron and vice versa, as shown in Figure 1. This tetrahedral coordination is characteristic of $\mathrm{sp}^{3}$ covalent bonding, but the bonds in $\mathrm{ZnO}$ have considerable ionic character, lying at the borderline between covalent and ionic. The wurtzite structure has a hexagonal unit cell with two lattice parameters, a and $\mathrm{c}$, in the ratio of $\mathrm{c} / \mathrm{a}=1.633 . \mathrm{ZnO}$ belongs to the space group of $\mathrm{P}_{3} \mathrm{mc}$ [29]. This structure is created by two interpenetrating hexagonal close packed sub-lattices, each of which consists of one type of atom displaced with respect to each other along the threefold $c$-axis by the amount of $u=(a / c)^{2}=0.375$ in fractional coordinates. The $\mathrm{ZnO}$ unit cell has a natural anisotropy which facilitates certain growth patterns, such as nanowire growth, which proceeds along the c-axis, and platelet growth, occurring along the hexagonal plane. The unit cell also has a net charge dipole moment along the $\mathrm{c}$-axis which makes $\mathrm{ZnO}$ piezoelectric. 


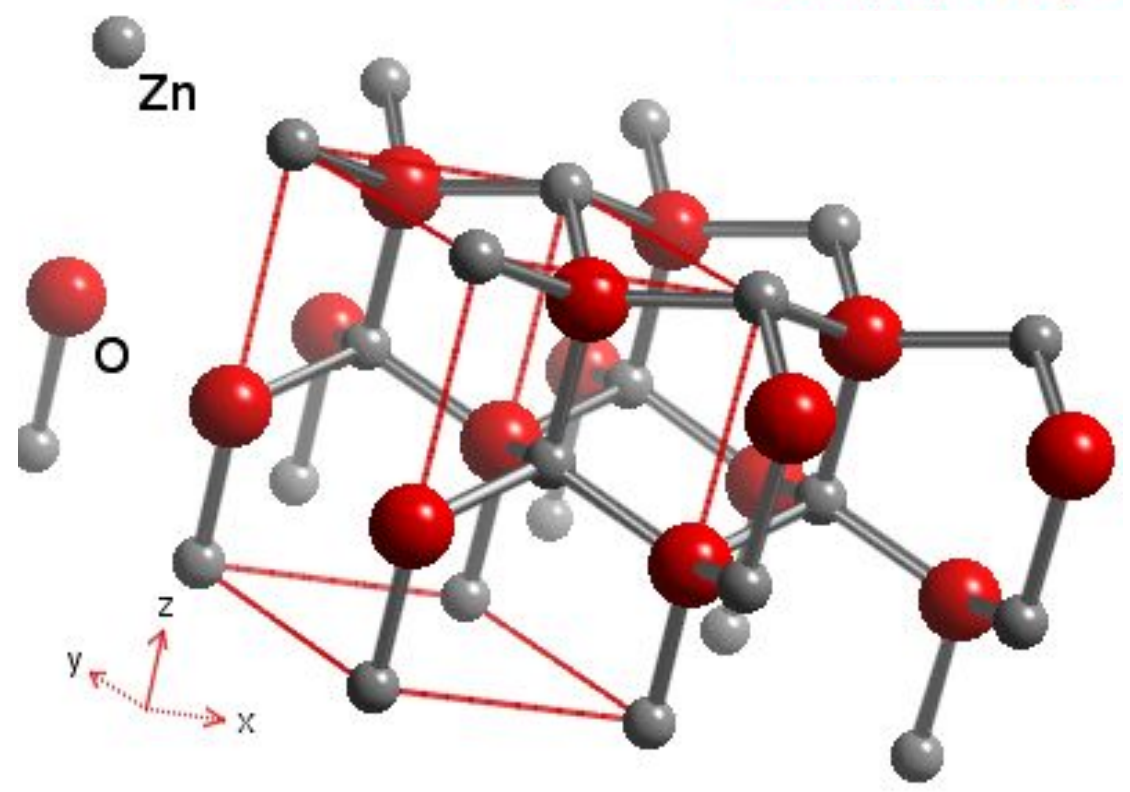

Fig. 1: Crystal structure of $\mathrm{ZnO}$ [39]

\subsubsection{2. $\mathrm{ZnO}$ electronic structure}

For most semiconductor device applications, a precise study of the band structure is needed. The electronic band structure of $\mathrm{ZnO}$ has been calculated by several groups [40-46]. A number of experimental studies have also been conducted regarding the band structure of the electronic states of wurtzite $\mathrm{ZnO}$. X-ray or UV reflection/absorption or emission methods have typically been used to measure the electronic core levels in solids. These techniques essentially measure the energy difference by inducing transitions between electronic levels or by exciting collective modes. The energy region can also be explored using a method based on the photoelectric effect which employs X-ray photons, i.e. X-ray photoelectron spectroscopy. More recently, researchers have started to use the angle-resolved 
photoelectron spectroscopy method with synchrotron radiation excitation. This method permits experimental bulk and surface electronic band structure determinations and is based on k conservation and a single nearly free electron-like final band [47]. The primary goals for this band structure investigation are to determine the bandgap and dispersion relationship $E(k)$ of $\mathrm{ZnO}$, as well as to understand the origin of both excitons and the formation of defects. The term "bandgap" refers to the energy difference between the top of the valence band and the bottom of the conduction band (See Figure 2 (a)). In order to excite an electron to transition from the valence band to the conduction band, a specific minimum amount of energy is required; this energy is referred to as bandgap energy. A diagram illustrating the band structure of $\mathrm{ZnO}$ is shown in Figure 2 (b). The wide bandgap of $\mathrm{ZnO}(3.37 \mathrm{eV})$ enables it for use in applications in optoelectronics in the blue/UV region, including light-emitting diodes, laser diodes, and photodetectors. $\mathrm{ZnO}$ also possesses a large exciton binding energy of $60 \mathrm{meV}$, indicating large bond strength and an extreme stability of excitons. This characteristic makes $\mathrm{ZnO}$ a promising material for the realization of efficient excitonic lasing at room temperature. 


\section{Conduction Band}

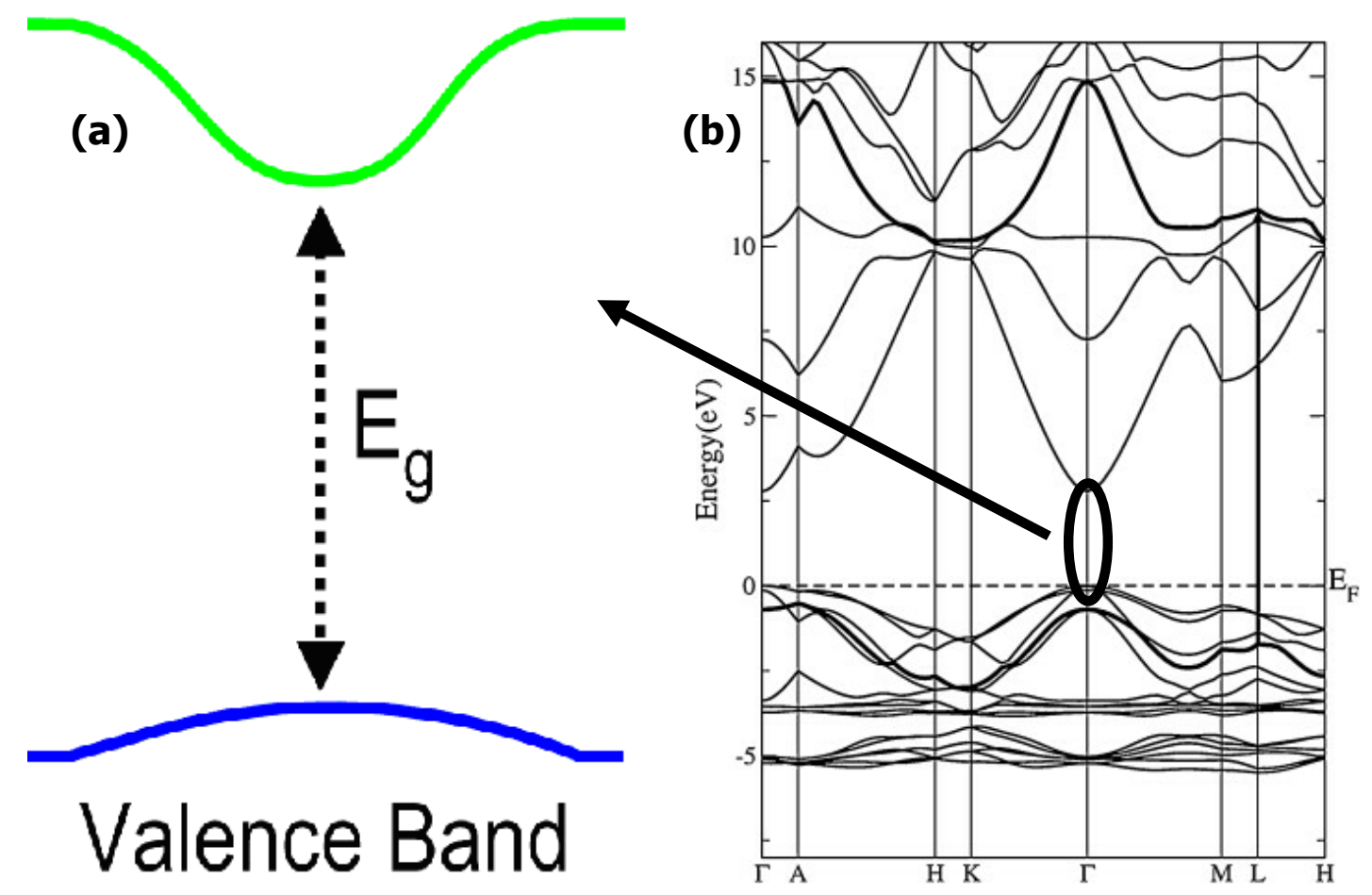

Fig. 2: Band structure of $\mathrm{ZnO}$ [209]

\subsubsection{Doping of $\mathrm{ZnO}$}

$\mathrm{ZnO}$ is inherently n-type due to the natural formation of negatively charged crystal defects, such as $\mathrm{O}$ vacancies and $\mathrm{Zn}$ interstitials. In order to exploit a broad range of device applications, it is necessary to alter the electronic properties of $\mathrm{ZnO}$ by impurity doping. The literature suggests that the incorporation of impurities into $\mathrm{ZnO}$ is still a contentious matter. This process can affect the structural, electrical, magnetic, and optical properties of $\mathrm{ZnO}$; however, a full understanding of its underlying mechanisms 
has not yet been obtained. $\mathrm{ZnO}$ is usually synthesized by various vapor deposition processes, which require costly high temperature and ultrahigh vacuum techniques. In contrast, doping of $\mathrm{ZnO}$ with metal ions by electrodeposition as carried out in this study is a low-cost and large-area method to create high crystalline quality nanostructures. Doping of $\mathrm{ZnO}$ thin films and $\mathrm{ZnO}$ nanowires with metal ions via electrodeposition has been discussed by various groups, including our studies [48-54]. The growth of low resistivity $\mathrm{ZnO}$ with p-type conductivity is still difficult due to the low solubility of extrinsic dopants and the self-compensation of intrinsic donors. P-type doping of $\mathrm{ZnO}$ has been reported, but it remains controversial. So far, most of the p-type doping techniques have been carried out by various vapor deposition processes. However, growth and doping of $\mathrm{ZnO}$ via an aqueous solution route is an attractive alternative due to its cost effective large scale synthesis. Only one group's limited work on low temperature electrochemical p-type doping has been reported [55-57], while no other successful reports of $\mathrm{p}$-doping in $\mathrm{ZnO}$ by electrodeposition have been produced.

\subsubsection{Defects in $\mathrm{ZnO}$}

$\mathrm{ZnO}$ is an important material for fabrication of optoelectronic devices on account of its direct and wide bandgap. There has been considerable effort expended toward developing $\mathrm{ZnO}$ nanostructures, since controlling the size and dimensions can lead to unique optical and electronic properties. This direct band gap nanostructure can be characterized by near bandgap luminescence studies, but this is not often the case where emission is dominated by low energy luminescence bands caused by defects. 
These defects introduce energy levels within the bandgap of $\mathrm{ZnO}$ [58]. It is important to understand that $\mathrm{ZnO}$ has a relatively open structure with a hexagonal close-packed lattice where $\mathrm{Zn}$ atoms occupy half of the tetrahedral sites. Hence, there are plenty of sites for $\mathrm{ZnO}$ to accommodate intrinsic defects and extrinsic dopants. The most common defects in $\mathrm{ZnO}$ are zinc interstitials and oxygen vacancies [59]. Oxygen vacancies are present as deep level donors in $\mathrm{ZnO}$, whereas $\mathrm{Zn}$ interstitials are shallow donors in $\mathrm{ZnO}$. Optically detected magnetic resonance (ODMR) was used to identify these point defects [60]. The electrical and optical properties of $\mathrm{ZnO}$ can be altered by these point defects.

\subsubsection{Magnetic properties of $\mathrm{ZnO}$}

For over a decade, diluted magnetic semiconductors (DMSs) have been studied due to their potential applications in spintronics. Much of the research on diluted magnetic semiconductor materials begins with incorporating the cations into III-V and II-VI compound semiconductors in the form of magnetic transition metal (TM) ions, such as $\mathrm{Mn}, \mathrm{Co}, \mathrm{Cu}$, and $\mathrm{Ni}$. The presence of the TM ions in the semiconductor leads to an exchange interaction between itinerant $s p$ band electrons and holes and the $d$ electrons localized at the magnetic ions, resulting in various magnetic field-induced phenomena, such as the anomalous Hall Effect and spin-dependent optoelectronics. Metal oxide semiconductors, such as $\mathrm{ZnO}$ and $\mathrm{TiO}_{2}$, have advantages over non-oxide semiconductors. They are typically wide bandgap semiconductors, transparent to visible light, highly n-type conductive, capable of growth at low temperatures on glass and 
plastic substrates, and safe, durable, and low-cost materials. The area of magnetic research on bulk, thin film, and nanostructured $\mathrm{ZnO}$ is still in an early stage, and several serious questions remain in need of clarification to better understand the development potential. The structural and the electronic characteristics of magnetic nanostructures have been studied in recent years because their characteristics can be tuned if the magnetic properties are controllably modified. A transition in the magnetic behavior from ferromagnetic to superparamagnetic can be achieved, as reported first by Yang et al., (2002) [61]. Superparamagnetic particles can be easily aligned with the external field even in weaker magnetic fields. The directions of these particles become random almost immediately after the field is turned off because the thermal energy has an ability to flip the dipoles in random directions. Thus, the benefit of achieving superparamagnetism is that there is no memory effect in the device. The material becomes magnetic only when a magnetic field is applied. This is what makes superparamagnetic particles so useful, especially in biomedical applications. Chen et al., 2008 [62] reports that superparamagnetism can be obtained by substitution of transition-metal-ions in one-dimensional (1D) semiconductor nanowire lattice sites. Our own work shows that at room-temperature the $\mathrm{Co}, \mathrm{Mn}$, and $\mathrm{Cu}$ doped $\mathrm{ZnO}$ nanowires are superparamagnetic, while, as expected, there is no measurable magnetic response from the undoped and Al-doped nanowire film. 


\subsubsection{Overview of $\mathrm{ZnO}$ nanostructures}

Presently, extensive research efforts are being directed towards the creation of $\mathrm{ZnO}$ nanostructures, the study of their growth mechanisms, and the investigation of their structural, optical, and electronic properties. With its anisotropic unit cell structure, $\mathrm{ZnO}$ can produce nanoparticles with high aspect ratios (length to width ratio), which are attractive for many optoelectronic applications. These nanostructures can be synthesized in a variety of processes, including chemical vapor deposition [63], metalorganic vapor phase epitaxy [64], electrodeposition [65, 66], and electroless solution deposition [67]. The growth of $\mathrm{ZnO}$ nanowires by electrodeposition is attractive in part because of its low-cost and low-temperature method for growing $\mathrm{ZnO}$ nanowires on conductive transparent electrodes. This method of $\mathrm{ZnO}$ growth was introduced in the mid-1990s $[68,69]$, followed immediately by a detailed article outlining the growth technique [65]. The initial growth study by Lincot et al., 1996 reports that the growth of dense films or nanowire arrays was controlled by tuning the zinc concentration in the solution [68]. The high zinc concentrations and an excess of the oxygen concentration lead to dense thin film growth while low zinc concentrations result in the formation of nanowires. $\mathrm{ZnO}$ nanostructures are a versatile and functional material that has a diverse group of growth morphologies such as nanobelts [70], nanowires [66], nanosprings [71], nanorings [72], and nanohelies [73], and it is most likely the richest family among all nanostructured materials (See Figure 3). These nanostructures present potential for significant applications, particularly in biosensing devices and other nanoscale

transducers. While it is well established that $\mathrm{ZnO}$ can be doped up to $2 \times 10^{21} \mathrm{~cm}^{-3}$ by 
introducing heavy substitutional impurities [29]. Various research studies have been conducted on the fabrication of transition metal doped $\mathrm{ZnO}$ nanowires using different techniques [75-78]; doping plays a role as a diluted magnetic semiconductor for spintronics applications. Most recently, Cui et al. [79] reported a route to p-type doped $\mathrm{ZnO}$ nanowires with the incorporation of $\mathrm{Ag}$ by low-temperature electrochemical growth followed by annealing. However, these results have not been confirmed by other groups.

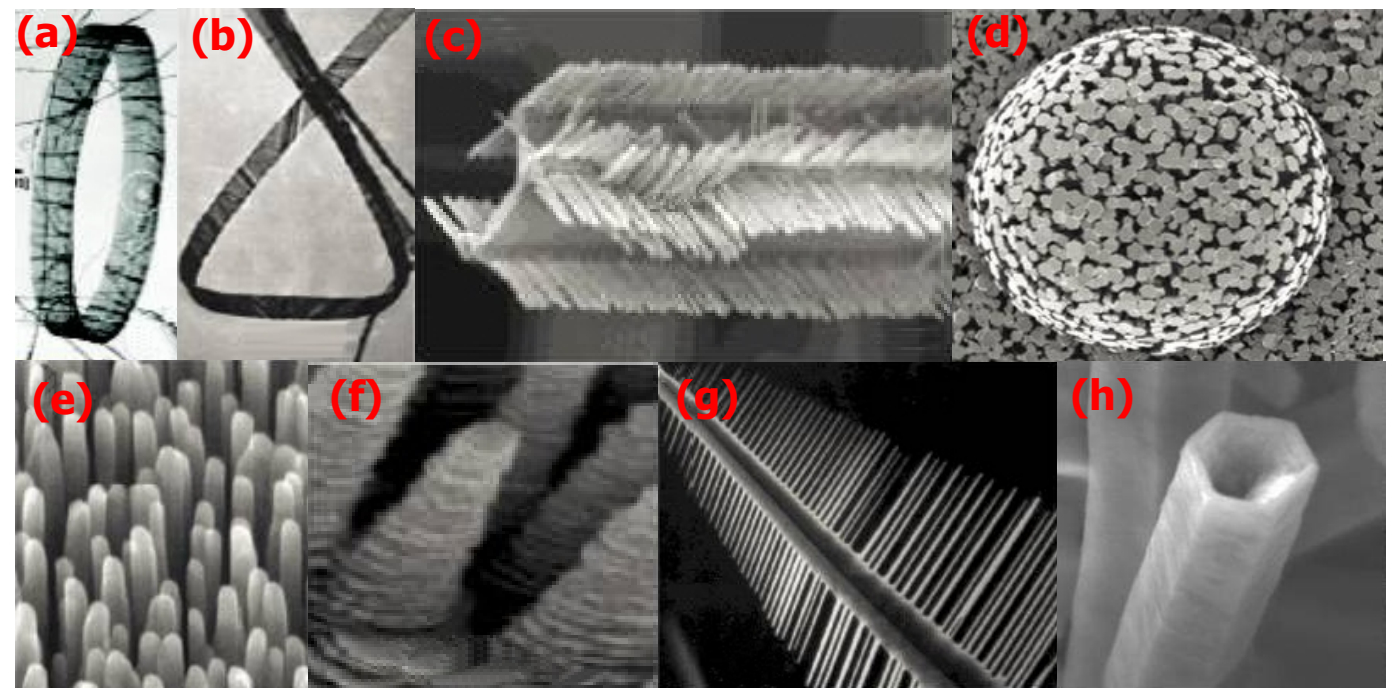

Fig. 3: Various forms of $\mathrm{ZnO}$ nanostructures: (a) nanoring, (b) nanoblet, (c) nanobridge, (d) nanoflower, (e) nanowires, (f) nanohelies, (g) nanocomb, and (h) nanotube [80] 
2.1.4 Our research on $\mathrm{ZnO}$ material properties and growth

In this work, we report a detailed study of the electrical, optical, magnetic, and structural properties of $\mathrm{ZnO}$ nanowires grown from $\mathrm{Zn}^{2+}$ solution using a lowtemperature electrodeposition process, the incorporation of impurities introduced during the growth process, and the annealing behavior of all types of properties. Doping of the $\mathrm{ZnO}$ nanostructures is achieved electrochemically using soluble chloride compounds as precursors. Growth on solid and flexible substrates, selective growth on n- and p-type $\mathrm{Si}$ substrates has also been explored by us [12, 13, 81 and 82]. Effects of impurity incorporation into the nanowires have been addressed [83, 84], and it appears that lowtemperature electrochemical growth is a unique method for incorporating conveniently large amounts of impurities into nanowire structures. Temperature-dependent photoluminescence showed that these impurities can be electronically activated at moderate temperatures. The room-temperature magnetization of $\mathrm{ZnO}$ nanowire films doped with transition metal ions has also been studied at PNNL [85]. The roomtemperature magnetic measurements show that the $\mathrm{Co}, \mathrm{Mn}$, and $\mathrm{Cu}$ doped $\mathrm{ZnO}$ nanowires are superparamagnetic; as we expected, there is no measurable magnetic response in undoped and Al-doped nanowire films. Moreover, we have reported for the first time visible and ultraviolet electroluminescences from $\mathrm{ZnO}$ nanowire/polymer devices in the course of our study, and we have also demonstrated $3.4 \%$ conversion efficiencies in nanowire-quantum-dot solar cells [12, 13, 81, 86 and 87]. Furthermore, we observed the improvement of the optical and structural properties of $\mathrm{ZnO}$ nanowires as a result of thermal and laser annealing after the electrodeposition process. The as- 
grown nanowires are rich in strain-related defects. Moderate thermal annealing as well as pulsed laser annealing, however, leads to strain relaxation and thus the formation of point-defects, as evidenced by a combination of high-resolution transmission electron microscopy (HRTEM) and electron diffraction experiments on single wires [83].

\subsection{Light emitting diodes (LEDs)}

The current goal in optoelectronic engineering is to replace conventional lighting sources such as incandescent and fluorescent lighting with more power efficient and low-cost semiconducting light sources. They have already had an impact in our daily life. A major advantage of LED lighting is that it reduces the ecological footprint on our environment. Through a lower monitory expenditure on energy, selfsustainability, and lower fire risk in powering these devices, LEDs become a low-cost alternative to conventional light sources.

\subsubsection{Overview of light-emitting-diodes (LEDs)}

The cubic II-VI (MgZnCdSe) compound semiconductor became the first efficient light emitting diodes (LEDs) and laser diodes (LDs), operating in the blue/green spectral range [88]. These devices showed excellent optical and electrical characteristics $[88,89]$; however, they failed to achieve long-term stability due to the high mobility of native point defects in the II-IV semiconductors. There were 
significant studies launched toward improving the lifetime of the II-VI light emitters, but many issues are yet to be solved.

\subsubsection{Development of III-nitride based light-emitting diodes}

III-nitride LEDs have replaced II-VI devices due to the breakthrough in InGaN LED technology that occurred in the early 90s $[90,91]$. The AlGaInN have now become the standard materials for blue/green light emitters. The nitride based LEDs provided a superior device performance as well as a long lifetime for useful industrial applications. Recently, the spectral response of these LEDs has been extended to the near-UV [92, 93] and the deep-UV [94] ranges. These nitride epitaxial materials have an inherent problem with high dislocation density because of their lack of native substrates, such as GaN or AlN [95]. There have been significant studies toward reducing this dislocation density. However, the high dislocation density issues in the IIInitride heterostructures are presently yet to be completely resolved.

\subsubsection{Development of $\mathrm{ZnO}$ based light-emitting diodes}

In addition to current III-nitride LED developments, II-VI oxide compounds continue to receive great attention in the field of LED technology as they are inexpensive, relatively abundant, chemically stable, and easy to prepare. In particular, the direct wide-bandgap semiconductor of $\mathrm{ZnO}$ has gained much attention in this research area due to its ability to be grown using low-cost and low temperature solution- 
based synthesis methods. Furthermore, $\mathrm{ZnO}$ has excellent structural and physical properties when compared to other wide-bandgap semiconductors, such as GaN, the wide-bandgap semiconductor currently utilized in the short-wavelength optoelectronics industry. Some of these advantages include a larger exciton binding energy (60 meV), a higher radiation hardness, simpler processing due to amenability to conventional chemical wet etching, and the availability of large area substrates at relatively low material costs $[96,97]$. The combination of a large band gap and a high exciton binding energy makes $\mathrm{ZnO}$ an excellent candidate for creating $\mathrm{UV}$ and blue light emitting devices. The amount of excitons in $\mathrm{ZnO}$ at room temperature was estimated by Osinsky et al., 2006 [74]. Their calculations demonstrate that excitons in $\mathrm{ZnO}$ are screened when the thermal carrier density rises above $\sim 2 \times 10^{19} \mathrm{~cm}^{-3}$. However, under typical LED operation, the exciton density in $\mathrm{ZnO}$ ranges only from $10^{18}$ to $10^{19} \mathrm{~cm}^{-3}$, indicating that $\mathrm{ZnO}$ could be an ideal material to create highly efficient light emitters at room temperature. When synthesized on homoepitaxial substrates, LED devices using $\mathrm{ZnO}$ have shown a lower dislocation density as compared to those that utilize group-III nitrides. However, the potential for creating efficient $\mathrm{ZnO}$ LEDs is yet to be fully achieved due to a high LED series resistance and the lack of a reliable and stable pdoping. A number of research groups have demonstrated that one solution for overcoming the problems associated with $\mathrm{ZnO}$ p-doping is the use of hybrid heterostructures. 


\subsubsection{Progress of $\mathrm{ZnO}$ thin film based LEDs}

The use of materials that can be easily and reliably p-doped is a natural way to avoid the difficulty of making p-doped $\mathrm{ZnO}$. A number of such p-type materials have been studied previously to obtain $\mathrm{ZnO}$ based hybrid LED heterostructures. The first $\mathrm{ZnO}$ based hybrid heterostructure was demonstrated by Drapak et al., 1967 [98]. In their work, they used $\mathrm{Cu}_{2} \mathrm{O}$ as a p-type coating that was obtained by thermal oxidation of a $\mathrm{Cu}$ metal. When deposited on vapor phase grown $\mathrm{ZnO}$ substrates, the electroluminescence showed broad spectra with maxima at $540 \mathrm{~nm}$ in both forward and reverse biases. Tsurkan et al., 1975 [99] constructed a $\mathrm{ZnO}$ heterojunction LED using pZnTe. In their research, p-ZnTe/n-ZnO LEDs were fabricated with two different carrier concentrations of electron and hole. In both cases intense electroluminescence was observed, but the spectra varied for different carrier concentrations. The broad yellow band with maxima at 2.0 and $2.22 \mathrm{eV}$ was obtained from $\mathrm{p}-\mathrm{ZnTe} / \mathrm{n}-\mathrm{ZnO}$ heterostructure with an electron and hole concentrations of $9 \times 10^{17}$ and $2 \times 10^{17} \mathrm{~cm}^{-3}$, respectively. On the other hand, the LED structure with the respective electron and hole concentration of $5 \times 10^{14}$ and $8 \times 10^{18} \mathrm{~cm}^{-3}$ exhibited an intense narrow band in the violet region with a maximum at $3.02 \mathrm{eV}$ and a set of much weaker bands peaking at 2.8 and $2.34 \mathrm{eV}$. The electron injection from low-resistive $n$ - $\mathrm{ZnO}$ into high-resistive $p$ - $\mathrm{ZnTe}$ causes the yellow band emission in $p$ - $\mathrm{ZnTe} / n$ - $\mathrm{ZnO}$ heterostructures. The violet emission at 3.02 $\mathrm{eV}$ was explained by the authors as a result of donor-acceptor recombination. An ultraviolet emission was observed by Ohta et al., 2000 [100] from a pulsed-laser deposited $p-\mathrm{SrCu}_{2} \mathrm{O}_{2}$ and $n$ - $\mathrm{ZnO}$ heterostructure. This LED showed a diode-like 
behavior with a turn-on voltage of $\sim 3 \mathrm{~V}$, which agrees well with the bandgap energy of $\mathrm{ZnO}$. The room temperature electroluminescence exhibited a maximum at $382 \mathrm{~nm}$ under forward biases. The authors concluded the observed UV emission was due to a transition associated with the electron-hole plasma in $\mathrm{ZnO}$. To form a high quality heterostructure, the structural relationship between the semiconductors is a crucial factor because a lattice mismatch causes extended defects at the interface, which act mainly as nonradiative centers. Therefore, materials with close lattice parameters are necessary for fabrication of high-quality heterostructure devices. The semiconductor $\mathrm{GaN}$ has a very close lattice parameters to $\mathrm{ZnO}$ with a lattice mismatch of $1.8 \%$. As a result, the heterostructure created using $\mathrm{p}-\mathrm{GaN}$ and $\mathrm{n}-\mathrm{ZnO}$ is promising for high efficient light emitting applications. There have been significant studies focused on such heterostructure devices [101-103]. The growth, processing, and fabrication of $\mathrm{n}-\mathrm{ZnO} / \mathrm{p}$ GaN heterostructure have been reported by Alivov et al., 2003 [104]. In their work, the heterostructure were prepared by first growing a Mg-doped GaN film of thickness $1 \mu \mathrm{m}$ on $\mathrm{Al}_{2} \mathrm{O}_{3}(0001)$ by molecular-beam epitaxy, then by growing Ga-doped $\mathrm{ZnO}$ film of thickness $1 \mu \mathrm{m}$ by chemical vapor deposition on the $p$-GaN layer. This $\mathrm{n}-\mathrm{ZnO} / \mathrm{p}-\mathrm{GaN}$ heterostructure exhibited an electroluminescence spectrum with a maximum at $430 \mathrm{~nm}$ under forward bias conditions. The observed electroluminescence emerges from GaN when the electron is injected from the $n-\mathrm{ZnO}$ to the $p-\mathrm{GaN}$. 


\subsubsection{Progress of $\mathrm{ZnO}$ nanowire based LEDs}

However, these thin film based heterojunction LED devices have low efficiencies because of a high energy barrier at the p-n junction interface. This problem can be overcome by using $\mathrm{ZnO}$ nanowires in the fabrication of the LED device due to their high crystallinity, large surface area, and ability to produce quantum confinement. Moreover, nanowires can perform as direct waveguides, favoring light extraction without the use of lenses and reflectors. We were the first to report on the fabrication and characterization of a vertical $\mathrm{ZnO}$ nanowire-based LED formed on a glass/F: $\mathrm{SnO}_{2}$ substrate, in which all components of the device were created using solution-processed techniques [12]. Our device exhibits a narrow near-bandgap emission and a broad subbandgap emission at room-temperature [12, 13]. Jeong et al., 2006 [105] reported on the fabrication and characterization of $n-\mathrm{ZnO}$ thin film/ZnO nanowires/ $p-\mathrm{GaN}$ film heterojunction light-emitting diodes on $\mathrm{Al}_{2} \mathrm{O}_{3}$ substrates, where the $0.7 \mu$ m-thick plasma-enhanced molecular beam epitaxially grown Mg-doped p-GaN and MOCVD grown $\mathrm{n}-\mathrm{ZnO}$ nanowires had carrier concentrations of $1.2 \times 10^{18}$ and $2.4 \times 10^{18} \mathrm{~cm}^{-3}$, respectively. This LED device exhibited an electroluminescence with an emission peak at $386 \mathrm{~nm}$ when the diode was under forward bias conditions with a threshold voltage of $6 \mathrm{~V}$ and a maximum of electroluminescence at $12 \mathrm{~V}$. The LED performances degraded when the voltage became higher than $12 \mathrm{~V}$. It was concluded that this UV-emission originated from recombination within the $\mathrm{ZnO}$ nanowires. They also observed that thermally treating the device in hydrogen ambient led to an increase of electroluminescence as compared to the electroluminescence from an as-fabricated 
device. Recently, ultraviolet electroluminescence has been demonstrated by Lupan et al., 2010 [106] using epitaxially electrodeposited ZnO nanowires on a p-GaN substrate that exhibited a threshold emission voltage of $4.4 \mathrm{~V}$. Their device also showed a welldefined rectifying $\mathrm{I}-\mathrm{V}$ characteristic at room temperature. Ultraviolet emission with a peak wavelength near $397 \mathrm{~nm}$ was observed when the diode was forward biased and originated from exciton recombination within the $\mathrm{ZnO}$ nanowire.

\subsubsection{Our vertical nanowire LED research}

All solution-processed light emitting diodes offer a low manufacturing cost, large device areas, physical flexibility, and convenient means of integrating materials when compared with conventional epitaxially grown, lattice-matched, crystalline semiconductor devices. We successfully fabricated the first vertical all solutionprocessed nanowire LED structures, and our device performances are reported, in Könenkamp et al., 2004 [12]. In this thesis, we report on the all solution-processed $\mathrm{ZnO}$ /polymer based light emitting diodes with our primary focus on the fabrications and characterizations of $\mathrm{p}-\mathrm{i}-\mathrm{n}$ heterojunction light emitting diodes. These LEDs are fabricated by the electrodeposition of $\mathrm{ZnO}$ nanowires on a glass/F: $\mathrm{SnO}_{2}$ (FTO), insulated by polystyrene, coated by the hole-injecting polymer poly $(3,4-$ ethylenedioxythiophene) poly(styrenesulfonate) (PEDOT:PSS), and contacted with gold $[12,13]$. The observed electroluminescence (EL) spectra from as-grown and annealed vertically oriented electrodeposited $\mathrm{ZnO}$ nanowires LED structures both have an excitonic and a defect-related peak. These measurements clearly show the difference 
between doped and un-doped $\mathrm{ZnO}$ in the nanowires' optical properties. Our recent studies showed that the luminescence can be tuned by incorporating metal ions in the nanowires during their growth and followed by annealing [83]. We also successfully integrated such planar LED structures into flexible type LEDs by replacing the glass/F: $\mathrm{SnO}_{2}$ substrate with a PET/ITO film, obtaining similar results as compared to previous planar LED studies [81].

\subsection{Solar cells}

Solution-processed non-planar photovoltaic devices could offer a route for harvesting solar energy that is more economically viable because of their lowtemperature and low-cost roll-to-roll device fabrication, large-area coverage, and the capability of producing mechanically flexible devices. Our approach was to design a complete solar cell from solution-processed nanoparticles. Undoped $\mathrm{ZnO}$ thin film was deposited on a glass/F: $\mathrm{SnO}_{2}$ substrate by a spray pyrolysis technique at $300^{\circ} \mathrm{C}$ prior to $\mathrm{ZnO}$ nanowire growth. Aluminum-doped $\mathrm{ZnO}$ nanowire films were grown electrochemically using aqueous chloride-based electrolytes and soluble chloride compounds as precursors at temperatures below $90^{\circ} \mathrm{C}$. Al dopants were added to the electrolyte in the form of chlorine compounds. Compound semiconductor quantum dots of CdSe were synthesized using a wet chemical method and are employed here as an absorber material. The quantum dot suspensions underwent pyridine solution and 1,2ethanedithiol ligand exchanges and were subsequently deposited as thin films on $\mathrm{ZnO}$ nanowires. These deposited quantum dots were then converted into extremely thin 
continuous poly-crystalline films with typical grain diameters of $30-50 \mathrm{~nm}$ in a $\mathrm{CdCl}_{2}$ aided thermal anneal process. The back contact was a spin-coated hole-conducting material. The combination of nanowire growth, the quantum dots' surface treatment, quantum dot layer deposition, and the quantum dot layer's heat treatment, followed by optimization of these processes, produced solar cells with an open-circuit voltage up to $0.6 \mathrm{~V}$, a short circuit current of $\sim 15 \mathrm{~mA} / \mathrm{cm}^{2}$, an external quantum efficiency of $65 \%$, and an energy conversion efficiency of $3.4 \%$.

\subsubsection{Overview of nanostructured solar cells}

Nanostructured photovoltaic (PV) devices have gained substantial interest in the PV research community because of their potential for obtaining low-cost and large-area solar energy conversion [107]. In recent years, there have been significant efforts towards the use of solution-processed inorganic semiconductor quantum dots such as CdTe [108], CdSe [85, 109], $\mathrm{Cu}_{2} \mathrm{~S}$ [110], PbS [111-113], CuInSe 2 [114], and $\mathrm{CuInS}_{2}$ [115] as photon absorber materials in the nanostructured solar cell due to their advantageous properties. Firstly, their bandgap can be easily adjusted by changing the size of the particle to match the solar spectrum. Secondly, they can be deposited through a simple process as a very thin absorber on a deeply nanostructured sample, reducing the amount of materials needed for absorption. Moreover, solution-processed inorganic semiconductor quantum dots have been integrated into various solar cell designs, such as Schottky solar cells [116, 117], depleted heterojunction solar cells [118], extremely thin absorber solar cells [119], bulk heterojunction solar cells [120-122], inorganic- 
organic heterojunction solar cells $[123,124]$, and quantum dot sensitized solar cells $[125,126]$. Furthermore, multiple exciton generation (MEG) has been investigated using quantum dots, such as $\mathrm{PbS}$ [127], PbSe [128], PbTe [129], CdSe [130], and Si [131], in an effort to achieve a highly efficient solar cell. It is hoped that the use of quantum dots could enhance the efficiency of multiple exciton generation in quantum dot solar cells beyond the Shockley-Queisser limit of 32\% [132]. However, the realization of this multiple exciton generation process is yet to be achieved in solar cell research. In order to realize this phenomenon, ultrafast exciton dynamic studies as well as better device architectures are needed.

\subsubsection{Quantum-dot sensitized solar cells (QDSSCs)}

Nanostructured wide-bandgap semiconductor films offer a microscopic surface area that can be several orders of magnitudes larger than their geometric area. These films can also be sensitized with a thin absorber layer of quantum dots [133]. Quantum dot sensitized solar cells are an alternative to dye sensitized solar cells [134], which were first developed by Michael Grätzel and Brian O’Regan in 1991 [135]. The major difference between dye sensitized and quantum dot sensitized solar cells is the sensitizer that is employed to harvest the sun light. In the case of the dye sensitized solar cell, the sensitizer is an organic dye molecule or a metal-organic complex, while in the case of the quantum dot sensitized solar cell it is an inorganic semiconductor quantum dot. Wide bandgap metal oxide semiconductors, such as $\mathrm{ZnO}$ and $\mathrm{TiO}_{2}$, synthesized in the form of mesoporous films, nanorods, nanowires, and nanotubes, were used as a 
photoanode in the quantum dot sensitized solar cells in order to provide a large microscopic surface area for quantum dot deposition. The morphology of a mesoporous film consists of interconnected particles with diameters of several tens to hundreds of nanometers, which allow these films to enhance the optical path length by light scattering. To increase the conductivity of this device, either nanowires or nanorods were used as a photoanode due to the usually high electron mobility caused by their single-crystalline nature. Similarly, nanotubes composed of metal oxides were used to achieve the light trapping effect as well as to enhance the carrier transport. Generally, there are four different methods used to attach quantum dots onto wide band-gap metal oxide semiconductors: (i) chemical bath deposition (CBD) [136], (ii) successive ionlayer adsorption and reaction (SILAR) [137], (iii) ion layer gas reaction (ILGAR) [138], and (iv) using a bifunctional linker [139]. Among these processes, the chemical bath method is the simplest technique for the deposition of quantum dots on metal oxide surfaces. However, chemical bath deposition does not permit a precise control over its deposited material's particle size. Recently, the chemical bath method has been modified into a more successful technique referred to as Successive Ion-Layer Adsorption and Reaction (SILAR). The integration of semiconductor quantum dots, such as CdS [140], CdSe [141], CdS/CdSe [142], and InP [143], by either chemical bath deposition or a SILAR process, has been actively studied in this research area. ILGAR is another vacuum-free technique, which is capable of enabling the low-cost deposition of metal sulfide layers (for example $\operatorname{In}_{2} \mathrm{~S}_{3}$ and $\mathrm{ZnS}$ ) in a homogeneous manner. This method involves a two-step cyclic process, including the application of a precursor solution on a substrate by either dipping (Dip-ILGAR) or spraying (Spray-ILGAR), and 
conversion of the small amount of coated precursor salt solution into a metal sulfide in an $\mathrm{H}_{2} \mathrm{~S} / \mathrm{Ar}$ atmosphere, at an elevated temperature. The deposition of very thin $\operatorname{In}_{2} \mathrm{~S}_{3}$ layers by spray-ILGAR has been developed for the fabrication of a Cd-free absorber layer in nanostructured solar cells [138]. The linker assisted self assembly of quantum dots is another well recognized method by which quantum dots are attached onto nanostructured metal oxide surfaces through linker molecules. During this linker assisted self assembly, the nanostructured metal oxide sample is immersed in a bifunctional molecular linker solution of $(\mathrm{COOH})-\mathrm{R}-\mathrm{SH}$, where $\mathrm{R}$ is the organic core of the linker. The carboxyl group attaches to the nanostructured metal oxide film, while the thiol remains free to connect to the quantum dot. The modified film is then immersed in the quantum dot solution for several minutes to allow for the adsorption of quantum dots onto the metal oxide substrate. This procedure also typically involves fractional ligand exchange. In our recent solar cell research, we have utilized 1,2ethanedithiol (EDT) as a linker molecule to attach CdSe quantum dots onto the $\mathrm{ZnO}$ nanowire surface. Recently, Chen et al., 2009 [144] demonstrated that pre-synthesized CdSe quantum dots can be successfully attached onto a $\mathrm{TiO}_{2}$ substrate using a mercaptopropionic acid linker. Under one sun illumination, their CdSe quantum dot sensitized device exhibited a power conversion efficiency of $1.19 \%$. However, further research towards modifying the design of this type of solar cell is needed in order to achieve improved efficiencies. 


\subsubsection{Extremely Thin Absorber (ETA) solar cells}

This section focuses on the Extremely Thin Absorber (ETA) solar cell, which is a novel type of solid state solar cell. The main component of an ETA solar cell is its nanostructured geometry, permitting a considerable reduction of absorber material, the capability of utilizing inexpensive processes in fabrication, and an increased tolerance for non-optimum electronic properties. This solar cell consists of a thin layer of an intrinsic absorber material sandwiched between an n-type transparent material and a ptype material. The central goal of this solar cell design is the realization of a short collection path length for the photogenerated carriers. The geometry of this solar cell also provides a longer optical path for the incoming photons and ensures a maximum absorption of light due to the total thickness of the absorber. The extremely thin absorber concept was first proposed by Könenkamp's research group at the HahnMeitner Institute in Berlin. Ernst et al., 2001 [145] reported the construction of $\mathrm{TiO}_{2} / \mathrm{CdTe}$ based ETA solar cells with a detailed functional explanation for this type of device. Their solar cells exhibited a photovoltage between 600 and $700 \mathrm{mV}$ and a photocurrent density of 5 to $9 \mathrm{~mA} / \mathrm{cm}^{2}$ under AM1.5 illumination. Two years later, they demonstrated an improved solar cell with a short-circuit current density of above 15 $\mathrm{mA} / \mathrm{cm}^{2}$ from $\mathrm{Hg}$ doped $\mathrm{CdTe}$ on highly porous $\mathrm{TiO}_{2}$ substrates [146]. Since then there have been significant studies towards the development and improvement of various types of ETA solar cells [147]. The primary focus has been to explore the use of different absorber materials to enhance absorption and create low-cost devices. Researchers have also been investigating various highly nanostructured materials in an 
effort to improve charge transport. Historically, nanoporous $\mathrm{TiO}_{2}$ [148] and $\mathrm{ZnO}$ [149] nanowires have been used as n-type, highly nanostructured materials for the electron collecting window layer in ETA solar cells. The use of $\mathrm{Cu}_{2} \mathrm{~S}$ [150], $\mathrm{CuInS}_{2}$ [151], CdS [152], CdTe [152], CdSe [153], and $\mathrm{Cu}(\mathrm{In}, \mathrm{Ga}) \mathrm{S}_{2}$ [154] as absorber materials, as well as $\mathrm{ZnTe}$ [155], $\mathrm{CuSCN}$ [156], and $\mathrm{CuAlO}_{2}$ [157] as hole-conducting materials for the ETA solar cell has also been explored. Recently, Levy-Clémént et al., 2005 [156] reported an ETA solar cell composed of $\mathrm{ZnO} / \mathrm{CdSe} / \mathrm{CuSCN}$. Their fabricated device showed a power conversion efficiency of $2.3 \%$, with a short-circuit current density of 4 $\mathrm{mA} / \mathrm{cm}^{2}$, and an open circuit-voltage of $500 \mathrm{mV}$ under $360 \mathrm{~W} / \mathrm{m}^{2}$ illumination. An ETA solar cell was also demonstrated by Belaidi et al., 2008 [158], who used the ILGAR method to deposit $\operatorname{In}_{2} \mathrm{~S}_{3}$ as an absorber layer onto electrodeposited $\mathrm{ZnO}$ nanowires. Under $50 \mathrm{~mW} / \mathrm{cm}^{2}$ illumination, the constructed device exhibited a short-circuit current density of $2.8 \mathrm{~mA} / \mathrm{cm}^{2}$, an open circuit-voltage of $450 \mathrm{mV}$, and an overall power conversion efficiency of $3.4 \%$. Mesoporous $\mathrm{TiO}_{2}$ substrates are, like $\mathrm{ZnO}$ nanowires, also considered an ideal n-type highly nanostructured material. An ETA solar cell consisting of $\mathrm{Sb}_{2} \mathrm{~S}_{3}$ quantum dots on mesoporous $\mathrm{TiO}_{2}$ demonstrated a short circuitcurrent density of $14.1 \mathrm{~mA} / \mathrm{cm}^{2}$, an open circuit-voltage of $0.49 \mathrm{~V}$, and a power conversion efficiency of 3.3\% under one sun illumination, as reported by Itzhaik et al., 2009 [159]. Their device exhibited an excellent stability in air due to the formation of an $\mathrm{Sb}_{2} \mathrm{~S}_{3}$ layer on top of the $\mathrm{Sb}_{2} \mathrm{~S}_{3}$ quantum dots when exposed to air. Following the conceptual model of the extremely thin absorber solar cell, we also demonstrated p-i-n heterojunction devices consisting of $\mathrm{ZnO}$ nanowires and $\mathrm{CdSe}$ quantum dots as their main components. An energy conversion efficiency of $3.4 \%$ was obtained from our 
device under $85 \mathrm{~mW} / \mathrm{cm}^{2}$ illumination. This $3.4 \%$ efficiency is among the best efficiencies for this type of solar cell. To date, experimental works have achieved below $4 \%$ power conversion efficiency from this type of solar cell. However, theoretical studies performed by Taretto and Rau, 2005 [160] projected a potential 15\% power conversion efficiency from ETA solar cells. If this proposed efficiency can be achieved, the ETA solar cell would become a cost-efficient alternative to existing solar cells.

\subsubsection{Inorganic-organic heterojunction solar cells}

Semiconductor quantum dots have recently been combined with inorganicorganic heterojunction devices; such devices could provide a new and enhanced method of generating solar-to-electrical conversion [161]. This inorganic-organic heterojunction device functions by means of a very similar operational principle to that of ETA solar cells. Chang et al., 2010 [161] reported the most efficient solar cell of this type, using $\mathrm{Sb}_{2} \mathrm{~S}_{3}$ quantum dots as a sensitizer, $\mathrm{P} 3 \mathrm{HT}$ as a hole transporting polymer, and mesoporous $\mathrm{TiO}_{2}$ as an electron collecting window layer. Their device exhibited a maximum incident-photon-to-current efficiency (IPCE) of $80 \%$, and a power conversion efficiency of 5.13\% under air-mass 1.5 global (AM 1.5G) illumination with an intensity of $100 \mathrm{~mW} / \mathrm{cm}^{2}$. Kniprath et al., 2009 [162] also demonstrated inorganicorganic heterojunction solar cells consisting of $\mathrm{TiO}_{2}$ films sensitized by colloidal CdSe and CdTe quantum dots, and a hole transport layer of the conjugated polymer poly $(9,9-$ dioctyl-fluorene-co-N-(4-butylenphenyl)diphenylamine). Their fabricated devices showed low photo-response properties due to the poor interface between CdSe quantum 
dots and $\mathrm{TiO}_{2}$. The device also showed a short-circuit current density of $0.05 \mathrm{~mA} / \mathrm{cm}^{2}$, an open circuit-voltage of $1.0 \mathrm{~V}$, and an energy conversion efficiency of $0.015 \%$. These studies demonstrate that use of inorganic-organic device architecture has the potential to obtain a large-scale cost-efficient solar energy conversion; therefore, future research is needed in this area given the many unexplored combinations of quantum dot materials and hole conducting polymers.

\subsubsection{Our solar cell research}

In our solar cell study, we describe the preparation and properties of solar cells consisting of CdSe quantum dots, $\mathrm{ZnO}$ nanowires, and a p-type polymer layer, as their main components [86]. Based on the fabrication process used in creating the ETA solar cell, our CdSe quantum dot layer was deposited between the n-type transparent $\mathrm{ZnO}$ nanowires and a p-type polymer layer. The deposition of a well-covered thin absorber layer of CdSe quantum dots is favorable both for light absorption [164] and for charge separation [165], which are the two most critical steps in solar-to-electric energy conversion. All layers are made using cost-efficient electrochemical and solution deposition techniques. Prior to the $\mathrm{ZnO}$ electrodeposition process, a transparent conducting fluorine-doped $\mathrm{SnO}_{2}$ substrate is covered with a thin continuous layer of $\mathrm{ZnO}$ by spray pyrolysis, leading to a significant increase in solar cell performance by avoiding potential short-circuiting between substrate and p-type layer. A thin absorber layer, provided by the CdSe quantum dot film, conformally covers the verticallyoriented free-standing $\mathrm{ZnO}$ nanowires. Depending on annealing durations, the quantum 
dot layers can be converted from loosely connected particle arrangements to a continuous thin film of inter-connected quantum dots. A p-type layer is then applied to sandwich the CdSe layer between a p-n junction of $\mathrm{ZnO}$ nanowires and a p-type material. The back contact is a thermally evaporated Au layer. The combination of $\mathrm{ZnO}$, CdSe, and the p-layer allows for good absorption characteristics across most of the visible light spectrum. Furthermore, we investigated a number of variations to our solar cell design. These design variations included creating solar cells with different p-type layers, solar cells with or without application of a compact $\mathrm{ZnO}$ thin film prior to $\mathrm{ZnO}$ electrodeposition, and solar cells using various durations of CdSe quantum dot layer annealing. The power-conversion efficiency of our well-annealed nanowire-quantumdot-polymer solar cell ultimately reached $2.5 \%$ [87].

We also report on CdSe quantum-dot deposition using a layer-by-layer dipcoating that utilizes 1,2-ethanedithiol (EDT) as an efficient linking molecule for improving the electrical and photo-response properties of nanowire-quantum-dot heterojunction solar cells. EDT ligand exchange of the CdSe quantum dot provides energetically favorable charge transfer between the absorber layer and the adjacent layers. This observation suggests that the sulfur atom in EDT is efficiently bound to the quantum dot surface. Moreover, we find that the combination of EDT treatment followed by a shorter duration of CdSe quantum-dot film annealing results in an improved solar cell performance. Our goal is to reduce this CdSe annealing duration in order to replace the planar substrate with a flexible type substrate, which could provide new and enhanced solar cells. 
Furthermore, we explored a pyridine solution exchange of the as-synthesized CdSe quantum-dots initially suspended in toluene in order to create a well-assembled CdSe quantum-dot layer on our $\mathrm{ZnO}$ nanowires. The combination of the pyridine solution exchange, EDT ligand exchange, and surfactant aided thermal annealing of CdSe quantum dots further improved our present solar cell's performances, leading to a power-conversion efficiency of $3.4 \%$ with a short-circuit photocurrent density $\left(\mathrm{J}_{\mathrm{sc}}\right)$ of $15 \mathrm{~mA} / \mathrm{cm}^{2}$ and an open-circuit voltage $\left(\mathrm{V}_{\mathrm{oc}}\right)$ of $0.60 \mathrm{~V}$. 


\section{Experimental Methods}

\subsection{Growth techniques of $\mathrm{ZnO}$ nanowires}

This section describes the methods employed in the cleaning of substrates, preparation of chemical solutions, deposition of $\mathrm{ZnO}$ thin film, and low temperature solution synthesis of doped and undoped $\mathrm{ZnO}$ nanowires. The fluorine doped $\mathrm{SnO}_{2}$ coated glass, purchased from Pilkington Glass under the name "Tec 8", was used as a substrate. We used $\mathrm{KCl}$ as a supporting electrolyte and $\mathrm{ZnCl}_{2}$ as the precursor for $\mathrm{ZnO}$ by electrodeposition. Dopants were added to the electrolyte in form of chloride compounds. These substances, purchased from Sigma Aldrich, were reagent graded with $99.9+\%$ purity. The electrodeposition was carried out in an aqueous solution at concentrations of $\mathrm{ZnCl}_{2}$ ranging from $1 \times 10^{-4} \mathrm{M}$ to $5 \times 10^{-4} \mathrm{M}$, transitions metal ranging from $1 \times 10^{-6} \mathrm{M}$ to $20 \times 10^{-6} \mathrm{M}$, and the supporting electrolyte concentration of $0.1 \mathrm{M} \mathrm{KCl}$. This supporting electrolyte was introduced to assure a good conductivity of the solution. In an aqueous solution we carried out two different electrodepositions: (1) with transition metal ions and (2) without transition metal ions. In both cases, the potential was $-1 \mathrm{~V}$ versus the $\mathrm{Ag} / \mathrm{AgCl}$ (sat) reference electrode potential, the temperature was at $80^{\circ} \mathrm{C}$, and oxygen bubbling and a magnetic stirring were applied

We also synthesized $\mathrm{ZnO}$ nanowires in electroless solution growth. This process involved placing the substrate in a $50 \mathrm{mM}$ Zinc nitrate hexahydrate $\left(\mathrm{Zn}\left(\mathrm{NO}_{3}\right)_{2} \cdot 6 \mathrm{H}_{2} \mathrm{O}\right.$ and $50 \mathrm{mM}$ hexamethylenetramine for several hours at $90^{\circ} \mathrm{C}$. Prior to the deposition, 
spray pyrolysis was carried out to create a compact $\mathrm{ZnO}$ thin film on the FTO/glass substrate to act as a seed layer for the growth of the nanowires. After the resulting growth, the nanowire films were washed thoroughly with de-ionized water to eliminate residual salts and dried in nitrogen at room temperature.

\subsubsection{Electrodeposition}

Electrodeposition has received a great deal of attention from the viewpoint of its cost efficient, large-area, and low temperature process as well as soft processing of materials. It has been recently applied towards the creation of nanostructures and its operational principle is quite simple. Upon application of electric voltage, the positive metallic ions in the electrolyte will move toward the cathode, and the negatively charged ions will move toward the anode. Once the positive metallic ions reach the surface of the substrate (cathode), this negatively charged surface will provide electrons to reduce the positively charged ions into their metallic form. This migration of ions through the electrolyte constitutes the electric current in the external circuit. This technique is effective for creating monolayers and thin films on substrates that are conductive. It appears a well-suited growth mechanism for semiconducting oxides [166, 167], in most cases producing nanostructured materials. This technique can lead to $\mathrm{ZnO}$ nanowire growth, which was first demonstrated in 1996 [68, 69]. Tuning the zinc concentration in the solution, Lincot et al., (1996) were able to control whether the growth of dense films or nanowire arrays occurred [65, 69]. The high zinc concentrations and an excess of oxygen lead to dense thin film growth while low zinc 
concentrations resulted in the formation of nanowires. In this section, we present the basic aspects of electrochemical growth of undoped and transition metal ion doped $\mathrm{ZnO}$ nanowires. We successfully integrated such nanowires growth on planar substrates into flexible substrates by replacing the glass/F: $\mathrm{SnO}_{2}$ substrate by a indium tin oxide (ITO) coated polyethylene terephthalate (PET) film, taking advantage of the low temperature of this growth process [15].

\subsubsection{Standard electrodeposition}

Lincot et al., 1998 [170] describes in detail the process of growing $\mathrm{ZnO}$ nanowires by electrochemical method, which is a relatively cost-efficient and low temperature technique. In addition, the electrochemical method presents other advantages to alternative deposition techniques, such as the relatively uniform deposition obtained on substrates of complex shape and the higher deposition rate [168].

In the early stages of our work, undoped $\mathrm{ZnO}$ nanowire growth was achieved by the electrodeposition method from an aqueous solution composed of $3 \times 10^{-4} \mathrm{M} \mathrm{ZnCl}_{2}$ and $0.1 \mathrm{M} \mathrm{KCl}$ at $80^{\circ} \mathrm{C}$. The solution was prepared with the $99.999 \%$ reagent-graded chemicals of 0.04088 grams $\mathrm{ZnCl}_{2}$ and 7.4505 grams $\mathrm{KCl}$, and dissolved into one liter of de-ionized water. This solution was kept on the magnetic stirrer for $30 \mathrm{~min}$ in order to ensure the proper mixing of these chemicals. A fluorine doped $\mathrm{SnO}_{2}$ coated glass substrate was then cut into approximately $3 \mathrm{~cm} \times 3 \mathrm{~cm}$ pieces using a diamond tipped knife, a highly precise means of cutting these substrates. After being cut, these pieces 
were cleaned ultrasonically with methanol for $10 \mathrm{~min}$ and rinsed with de-ionized water. This ultrasonic cleaning process was then repeated using acetone for $10 \mathrm{~min}$ and rinsing with de-ionized water. Finally, the substrates were immersed into a $0.1 \mathrm{M} \mathrm{HNO}_{3}$ bath for 12 hours, cleaned with de-ionized water, and then dried with the $\mathrm{N}_{2}$ gas. The SEM microgram of cleaned fluorine doped $\mathrm{SnO}_{2}$ is shown below (Figure 4):

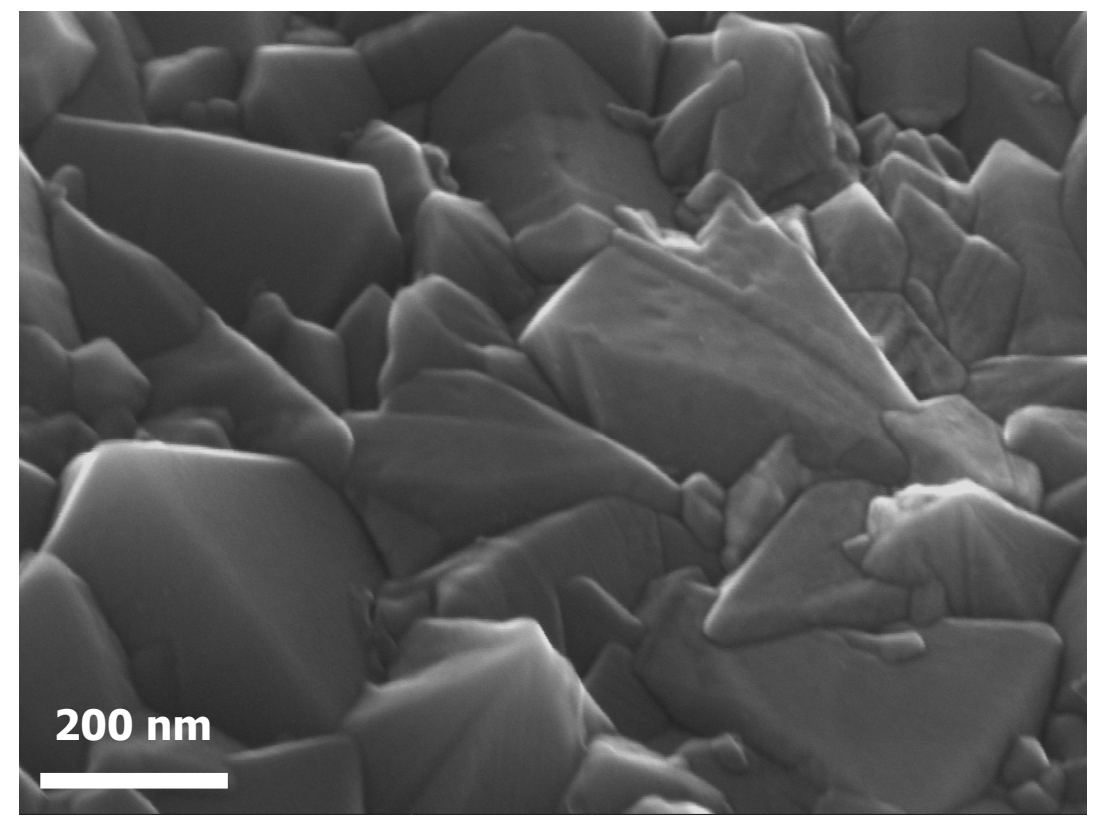

Fig. 4: SEM micrograph of transparent, fluorinated $\mathrm{SnO}_{2} /$ glass substrate

A classical three-electrode cell was used for all the electrochemical experiments. The working electrode consisted of a cleaned FTO substrate attached to a Pt wire by a paper clip. The counter electrode was a platinum foil, and all potentials were recorded with respect to a saturated $\mathrm{Ag} / \mathrm{AgCl}$ (sat) reference electrode. Prior to electrodeposition, the Pt counter electrode, the $\mathrm{Ag} / \mathrm{AgCl}$ (sat) reference electrode, and the paper clips were 
all rinsed with de-ionized water. In the meantime, the $\mathrm{Pt}$ working electrode was immersed in a cleaning vessel containing $0.5 \mathrm{M} \mathrm{HNO}_{3}$ and rinsed with de-ionized water. Using soap distilled water, the beakers, pipettes, thermometer, and magnetic stirrer were also cleaned and then rinsed several times with de-ionized water.

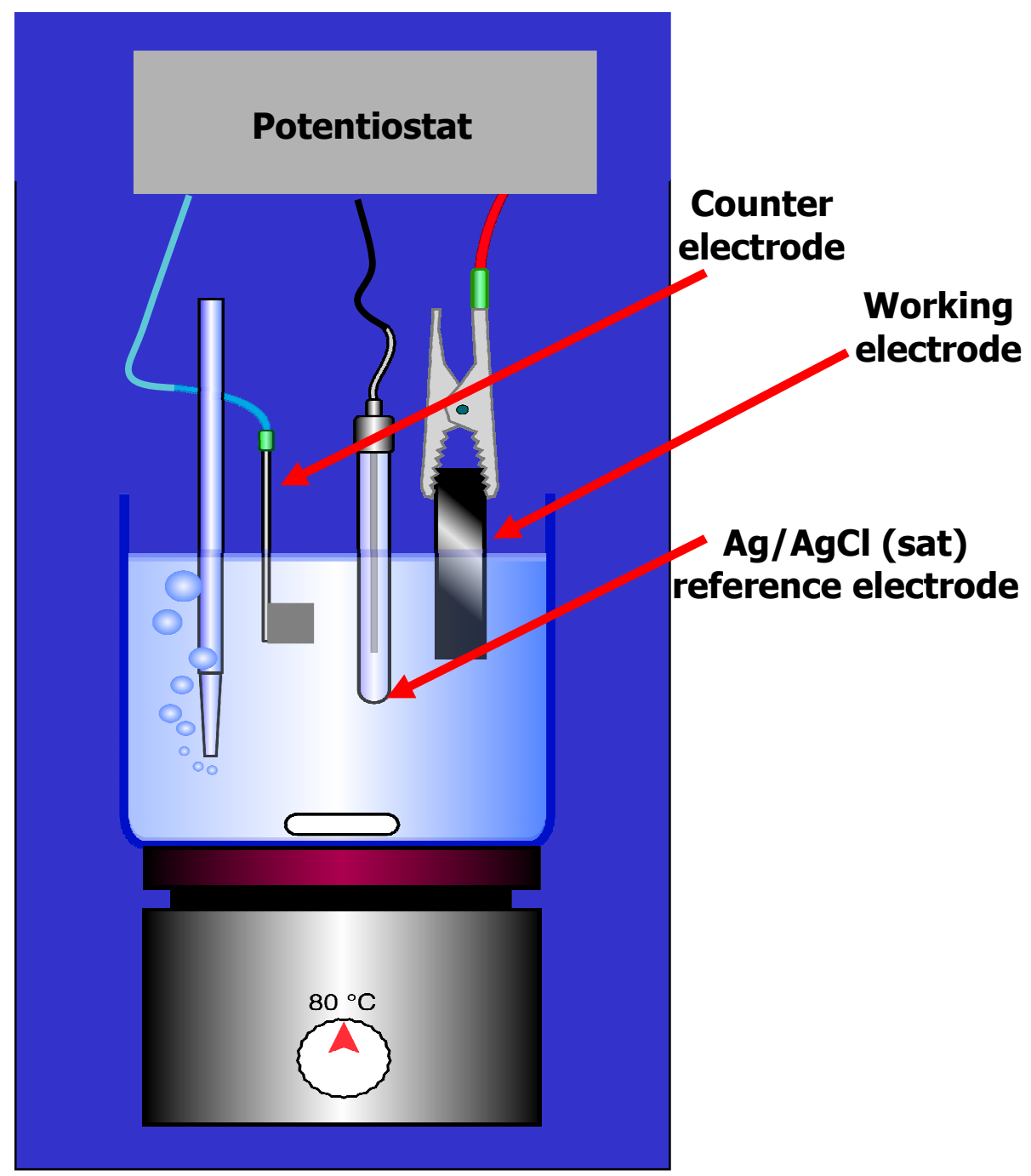

Fig. 5: Schematic diagram of electrodeposition setup 
The deposition solution was heated to $80^{\circ} \mathrm{C}$, under constant magnetic stirring, using a Vanlab DYLA-DUAL electric heater. Oxygen gas was then continuously bubbled into the electrodeposition solution in order to compensate for the loss of oxygen concentration caused by the reduction reaction that occurs throughout the deposition process. The electrodeposition system was controlled by a model 283 potentiostat, which was driven by the power step software, as shown in Figure 5. The $\mathrm{ZnO}$ nanowires were typically deposited at $-1.0 \mathrm{~V}$ versus $\mathrm{Ag} / \mathrm{AgCl}(\mathrm{sat})$. This deposition voltage of $-1.0 \mathrm{~V}$ was found to be the most appropriate voltage for optimizing the desired reaction. During the experiment, we maintained a constant temperature of $80^{\circ} \mathrm{C}$, and, in most cases, an hour long deposition. However, depositions shorter and longer than an hour were also performed. The $\mathrm{pH}$ of the solution was usually measured before and after deposition, with measurements before deposition ranging from 5 to $6 \mathrm{pH}$ and after deposition ranging from 4 to $5 \mathrm{pH}$.

Several different kinds of chemical reactions occurred during the deposition, supporting the transport of $\mathrm{Zn}^{2+}$ ions to the solution/substrate interface in order to achieve the following overall reactions.

The first reaction to occur was an oxygen reduction reaction, which is stated by:

$\mathrm{O}_{2}+2 \mathrm{H}_{2} \mathrm{O}+4 \mathrm{e}^{-} \rightarrow 4 \mathrm{OH}^{-} \quad \mathrm{E}_{\mathrm{o}}=0.4 \mathrm{~V} / \mathrm{NHE}$ 
Where $E_{o}$ is the standard electrode potential of the working electrode and NHE is the normal hydrogen electrode. In our case, the silver/silver-chloride $(\mathrm{Ag} / \mathrm{AgCl})$ was used as the reference electrode. The potential of this electrode is $+0.2 \mathrm{~V}$ at $298.15 \mathrm{~K}$ with respect to NHE.

In other words, this reaction causes the formation of hydroxide ions. Therefore, the $\mathrm{OH}$ - concentration around the working electrode increased. At the same time the following reaction occurred in the solution:

$\mathrm{ZnCl}_{2} \rightarrow \mathrm{Zn}^{2+}+2 \mathrm{Cl}^{-}$

These positively charged zinc ions drift through the solution towards the cathode, where they combine with negatively charged ions $\left(\mathrm{Cl}^{-}\right.$and $\left.\mathrm{OH}^{-}\right)$, producing soluble and insoluble species. The conditions of their formation are related to the chemical equilibrium:

$$
\begin{aligned}
& \mathrm{Zn}^{2+}+2 \mathrm{OH}^{-} \rightarrow \mathrm{Zn}(\mathrm{OH})_{2} \ldots \ldots \ldots \ldots . \\
& \mathrm{Zn}^{2+}+2 \mathrm{OH}^{-} \rightarrow \mathrm{ZnO}+\mathrm{H}_{2} \mathrm{O} \ldots \ldots \ldots . . \\
& \mathrm{Zn}^{2+}+\mathrm{x} \mathrm{OH}^{-}+\mathrm{y} \mathrm{Cl}^{-} \rightarrow \mathrm{Zn}(\mathrm{OH})_{\mathrm{x}} \mathrm{Cl}_{\mathrm{y}}
\end{aligned}
$$

The above reactions can be controlled by a few deposition parameters, such as temperature, potential, and $\mathrm{ZnCl}_{2}$ and $\mathrm{O}_{2}$ concentrations. In the presence of oxygen, the films are deposited on the substrate surface by the following reaction. 
According to this equation (6), the $\mathrm{Zn}^{2+}$ ions react with a single oxygen atom and form $\mathrm{ZnO}$ films at the substrate due to the electron injection from the underlying $\mathrm{SnO}_{2}$ layer. Thus, it can be anticipated that a reasonable electron contact at the $\mathrm{SnO}_{2} / \mathrm{ZnO}$ interface is established. After the deposition, the sample is rinsed carefully with de-ionized water and then dried with nitrogen at room temperature.

\subsubsection{Doping with transition metal ions}

The introduction of impurity atoms into semiconducting materials is the primary method for controlling the properties of the semiconductor, such as bandgap and electrical conductivity. This practice is performed routinely with bulk semiconductors and, more recently, has been extended to nanoscale semiconductors as well. II-VI compound semiconductors are doped with transition metals to study their novel magnetic properties and to develop dilute magnetic semiconductors as building blocks for spintronic devices. We used either $\mathrm{AlCl}_{3}, \mathrm{CuCl}_{2}, \mathrm{CoCl}_{2}$ or $\mathrm{MnCl}_{2}$ as a doping precursor. First, we prepared $1 \mathrm{mM}$ stock solution of these doping precursor solutions, and then took an appropriate volume of that stock solution and mixed it into the standard solution. The electrodeposition was carried out on fluorine doped $\mathrm{SnO}_{2}$ films on glass substrates in a standard three electrode deposition process at $80^{\circ} \mathrm{C}$ using an aqueous solution of $3 \times 10^{-4} \mathrm{M} \mathrm{ZnCl}_{2}, 0.1 \mathrm{M} \mathrm{KCl}$, and small micro molar amount of transition metal chloride. We added different concentrations of transition metal ion, 
such as 1, 3, 5, 10, 20 micro-molar concentrations. During all these depositions, we maintained $\mathrm{ZnCl}_{2}$ and $\mathrm{KCl}$ concentrations as a constant.

\subsubsection{Chemical bath deposition}

In addition to electrodeposition, we also explored the use of chemical bath deposition (CBD) as a means of growing vertically oriented $\mathrm{ZnO}$ nanowires on glass/F: $\mathrm{SnO}_{2}$ substrates. To begin this process, planar zinc oxide thin films were made using a spray pyrolysis method similar to that performed by O'Regan et al., 2000 [169]. These planar $\mathrm{ZnO}$ films serve as seed layers for the subsequent electro-less $\mathrm{ZnO}$ nanowire growth. A $0.05 \mathrm{M}$ solution of zinc acetate $\left(\mathrm{Zn}\left(\mathrm{CH}_{3} \mathrm{CO}_{2}\right)_{2}\right)$ in ethanol was made with a small amount of acetic acid $\left(\mathrm{CH}_{3} \mathrm{COOH}\right)$ to lower the $\mathrm{pH}$. Fluorine-doped tin oxide (FTO), silicon, or insulating glass was used as a substrate, which was placed on a glass slide on a hot plate to distribute the heat evenly. The substrate was heated to $300^{\circ} \mathrm{C}$ before the spraying began. The solution was applied using an Iwata Eclipse HPBCS airbrush, and thin films of varying thickness were created by changing the number of sprays, up to 45 sprays. The samples were then annealed for two hours at $380^{\circ} \mathrm{C}$.

The procedure used for growing $\mathrm{ZnO}$ nanowires on the planar films is based on the process described by Vayssieres et al., 2003 [174]. After the seed layer was formed by spray pyrolysis, the sample was submerged in a solution of $50 \mathrm{mM}$ zinc nitrate hexahydrate and $50 \mathrm{mM}$ hexamethylenetramine in de-ionized water at $90^{\circ} \mathrm{C}$ for three to 
four hours. It is important to note that we have not achieved any nanowire growth on a substrate without the present of the seed layer.

\subsection{Characterization techniques}

3.2.1 Scanning electron microscopy (SEM)

The surface morphologies of our fabricated nanostructures were analyzed at 20 $\mathrm{kV}$ with a spot size of three for imaging, and at $10 \mathrm{kV}$ with a spot size of five for elemental compositional analysis, using an FEI high resolution field emission scanning electron microscope (FESEM). The samples were mounted on SEM stubs using conducting carbon tape. As the glass substrate is not conductive, the charge effects limit the resolution of the SEM due to shifting of the electron beam. However, connecting the stub to the $\mathrm{SnO}_{2}$ substrate with $\mathrm{Cu}$ tape led to an observable improvement in the shifting. The SEM images were taken both normal to the surface and at angles of $45^{\circ}$ to the normal.

3.2.2 Transmission electron microscopy (TEM)

An FEI Tecnai F-20 field emission high transmission electron microscope (HRTEM), equipped with an energy dispersive X-ray (EDX) spectrometer, was utilized to characterize the structural properties of the $\mathrm{ZnO}$ nanostructures and to determine their chemical compositions. To prepare the HRTEM samples, $\mathrm{ZnO}$ was scratched from the 
substrate, dispersed in methanol, ultra-sonicated for five minutes, and then transferred onto a holey carbon TEM Cu grid using a micropipette. For electron diffraction pattern and high resolution image, the $\alpha$ and $\beta$ angles were adjusted to bring the sample in zone axis. The sample stage was tilted at an $\alpha$ angle of $15^{\circ}$ for STEM-EDX analysis. In order to further investigate the detailed nanostructure of $\mathrm{ZnO}$, both bright- and dark-field TEM images as well as electron diffraction patterns of a single nanowire were taken.

\subsubsection{Secondary ion mass spectrometry (SIMS)}

The technique of Secondary Ion Mass Spectrometry (SIMS) is the most sensitive of all the commonly employed surface analytical techniques. It is capable of detecting impurity elements present in a bulk concentration of $\sim 10^{15} \mathrm{~cm}^{-3}$. This is because of the inherent high sensitivity associated with mass spectrometric-based techniques. In our work, secondary ion mass spectrometry (SIMS) was used to measure the incorporation of doping concentration of the nanowires using $\left(\mathrm{O}^{2+}\right)$ sputtering analysis ions and $\left(\mathrm{Bi}^{+}\right)$primary analysis ions. The sputtering was carried out with an $\mathrm{O}^{2+}$ ion beam localized to an area of $1 \mu \mathrm{m} \times 1 \mu \mathrm{m}$.

\subsubsection{X-ray diffraction (XRD)}

The powder X-ray diffraction is the most common technique used to study the characteristics of crystalline structure and to determine the chemical composition and the crystal phase structure of the nanomaterials. Powder X-ray diffraction 
measurements were carried out in a Philips PW 3040 diffractometer using Co K $\alpha$ radiation, $2 \theta$ mode, and peltier-cooled energy dispersive detector. The lattice constants were determined using the Rietveld-refinement method.

\subsubsection{Vibrating sample magnetometer (VSM)}

A Vibrating Sample Magnetometer (VSM) was used to measure the magnetic behavior of magnetic materials. The VSM operates according to Faraday's law of induction: a changing magnetic field will produce an electric field. This electric field can be measured, and can therefore provide us with information about the changing magnetic field. In this work, the undoped and transition metal ion doped $\mathrm{ZnO}$ nanowires were grown on low conductivity n-type $\mathrm{Si}$ substrates by a low temperature electrochemical method. The magnetic measurements were performed using a LakeShore 7300 model VSM at room temperature. The $\mathrm{ZnO}$ nanowire film on the $\mathrm{Si}$ substrate was mounted on the VSM sample rod using vacuum grease, and the film was centered between two electromagnets. The magnetic field was applied parallel to the film surface and ramped from $10 \mathrm{kG}$ to $-10 \mathrm{kG}$ and back to $10 \mathrm{kG}$ over a period of 60 minutes. Separate experiments confirmed that the vacuum grease and $\mathrm{Si}$ substrate showed no detectable magnetic response. 


\subsubsection{Photoluminescence and laser annealing}

In our photoluminescence (PL) experiments, electrochemically grown $\mathrm{ZnO}$ nanowire films were studied before and after thermal annealing on doped and un-doped $\mathrm{ZnO}$ nanowires in the temperature range of $4 \mathrm{~K}-300 \mathrm{~K}$. A $325 \mathrm{~nm}$ wavelength of a HeCd laser was used as an excitation source and a photomultiplier tube with a GaAs photocathode was used to detect the PL signals. A closed-cycle He cryostat was used to control and monitor the temperature of the $\mathrm{ZnO}$ nanowire film between 4 and $300 \mathrm{~K}$. Various excitation powers were achieved by filtering the incident laser beam.

The laser annealing was performed using the third harmonic of a pulsed Nd:YAG laser with a wavelength of $355 \mathrm{~nm}$, pulse width of $10 \mathrm{~ns}$, pulse frequency of $10 \mathrm{~Hz}$, and pulse energy in the range of 10-40 mJ. Double-sided adhesive carbon tapes, rather than metal clamps, were used to hold the $\mathrm{ZnO}$ nanowire samples on the sample holder, thus eliminating unwanted luminescence generated by the metal clamps. The room-temperature photoluminescence experiments were performed in the same laser arrangement with a pulse energy of $2 \mathrm{~mJ}$, an energy level which is below the annealing threshold. The emitted photoluminescence from the $\mathrm{ZnO}$ nanowire films was detected using an Ocean Optics spectrometer with a grating of 11 . This grating has groove densities of $1800 \mathrm{~mm}^{-1}$ and an optical resolution of $0.72 \mathrm{~nm}$. 
3.3 $\mathrm{ZnO}$ nanowires/polymer based light-emitting diodes

\subsubsection{Overview}

The goal of the research was to obtain an efficient white-light emission from a hybrid LED arrangement using organic polymers and inorganic $\mathrm{ZnO}$ nanowire film. This was achieved by making diode structures consisting of electrodeposited $\mathrm{ZnO}$ nanowires sandwiched between a transparent fluorinated $\mathrm{SnO}_{2}$ film and a p-type conducting polymer [13]. Electroluminescence from these diodes was observed only when they were in forward bias conditions, but not in the case of reverse bias. This observation led us to conclude that the standard LED theory would be applicable when emitting the light under forward bias. While in forward bias, the gold pad acts as a positive terminal with the $\mathrm{SnO}_{2}: \mathrm{F}$ substrate acting as a ground terminal; however, in the reverse bias condition these terminals play an inverted role. We used a HewlettPackard 4145B semiconductor parameter analyzer in diode channel definition mode to measure the current-voltage (IV) characteristics of our LED devices. The electroluminescence spectra were measured using an Ocean Optics USB2000 Miniature Fiber Optic Spectrometer.

In order to construct the LEDs, the first important step is to coat $\mathrm{ZnO}$ nanowires with either low or high concentrations of insulating polystyrene. The polystyrene is applied in such a way that the space between nanowires is homogeneously filled with the insulator, while only a very thin exposure of insulator material occurs on the top of 
the nanowire. To achieve a better hole injection, we applied hole conducting polymer (PEDOT:PSS) layers on polystyrene covered $\mathrm{ZnO}$, leading to an improved emission intensity. The gold layers were then deposited on the top of the sample by vacuum evaporation in order to provide a Schottky barrier type contact for hole injection.

\subsubsection{Polystyrene coating}

The polystyrene deposition involves multilayer spin-coating techniques that utilize either high ( $1800000 \mathrm{~g} / \mathrm{mol})$ or low ( $280000 \mathrm{~g} / \mathrm{mol})$ molecular weight solutions of polystyrene in toluene. Depending on the nanowires growth densities, the lengths of the nanowires, and their diameters, various concentrations of polystyrene solution were used, such as two layers of $50 \mathrm{~g} / \mathrm{L}$ high molecular weight polystyrene solution, four layers of $50 \mathrm{~g} / \mathrm{L}$ low molecular weight polystyrene solution, or four layers of $30 \mathrm{~g} / \mathrm{L}$ high molecular weight polystyrene solution. We then imaged these different concentrations of polystyrene coating on $\mathrm{ZnO}$ nanowire film using SEM (See Figures 6, 7, and 8). 

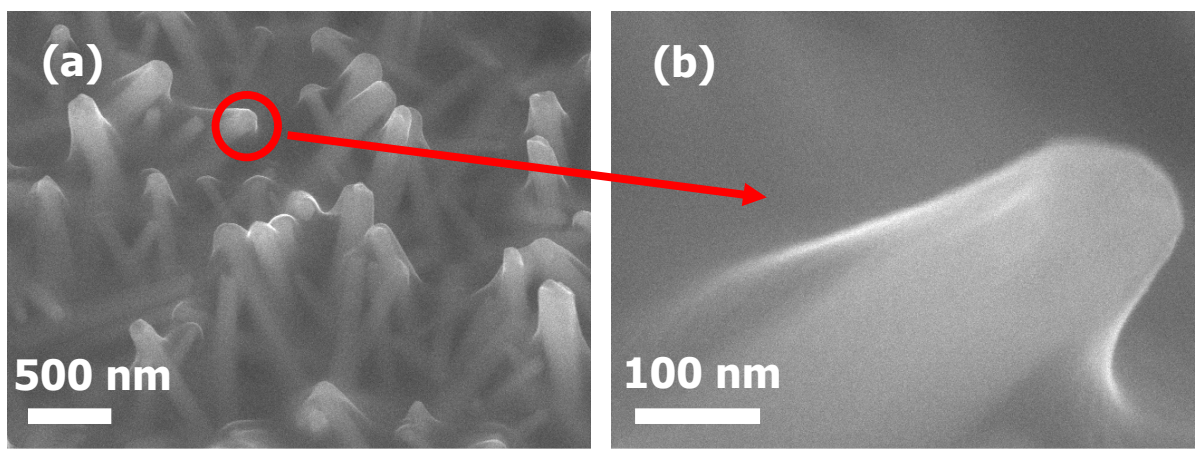

Fig. 6: SEM micrographs of (a) nanowires and (b) a single nanowire embedded in polystyrene (PS) solution of two layers of $50 \mathrm{~g} / \mathrm{L} \mathrm{HMW}$

SEM micrographs clearly show that the polystyrene layer homogeneously fills the space between the nanowires while creating only a very thin coating on the tops of the nanowires. The thickness of the polystyrene layer on the top of the nanowires appears to be in the range of $20-60 \mathrm{~nm}$, as is evident from the high magnification SEM micrograph in Figure 6 (a). The polystyrene acts as a very weak insulator at this thickness, therefore current transport occurs due to dielectric breakdown through electronic and structural defects. 

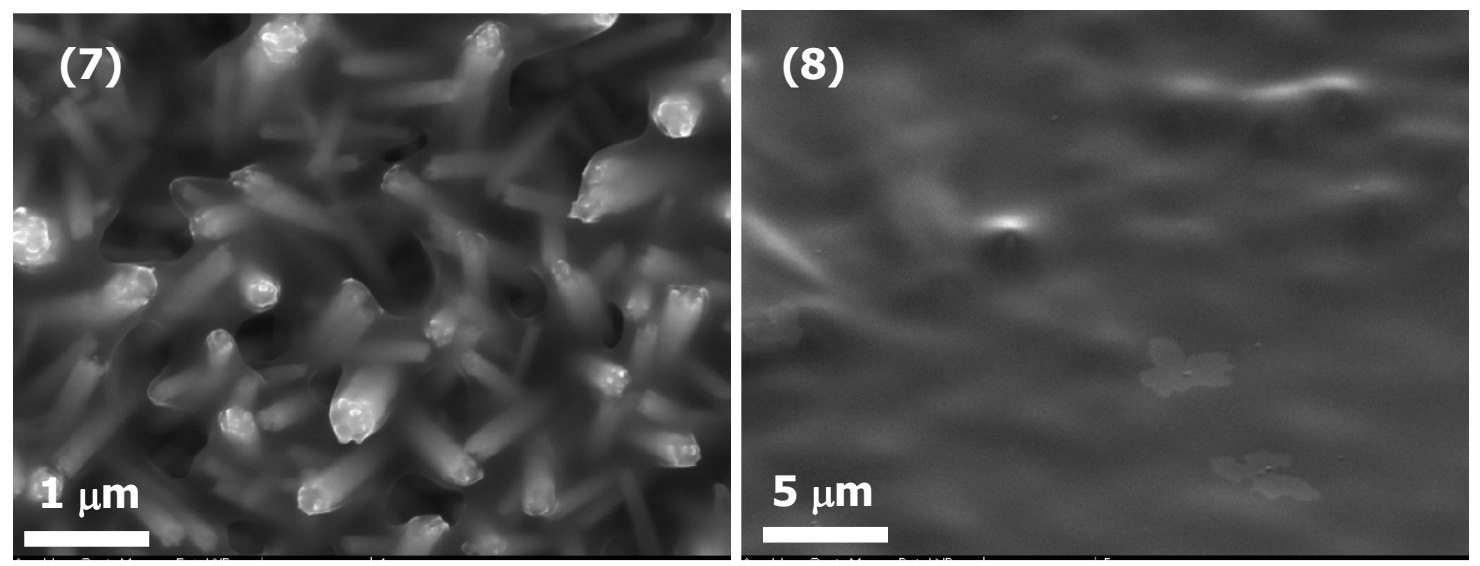

Figs. (7) \& (8): SEM micrographs of very thin polystyrene coating on $\mathrm{ZnO}$ nanowire film and very thick polystyrene coating on $\mathrm{ZnO}$ nanowire film, respectively

\subsubsection{PEDOT:PSS deposition}

The hole-conducting polymer PEDOT:PSS \{poly (3, 4-ethylenedioxythiophene)-poly (styrene-sulfonate) \} was then deposited on the sample in order to ensure a good electrical contact between the $\mathrm{ZnO}$ nanowires and anode surface. This polymer serves as hole injecting contacts. The PEDOT:PSS deposition was carried out manually in a paint-brush process, which gives a more homogeneous coverage on top of the polystyrene layer as compared to the spin coating process. The sample was then baked at $90^{\circ} \mathrm{C}$ for $5 \mathrm{~min}$, and allowed to cool to room-temperature prior to the next coating of PEDOT:PSS. After four coatings, the thickness of the PEDOT layer was in the approximate range of 200-300 $\mathrm{nm}$ as shown in Figure 9. 


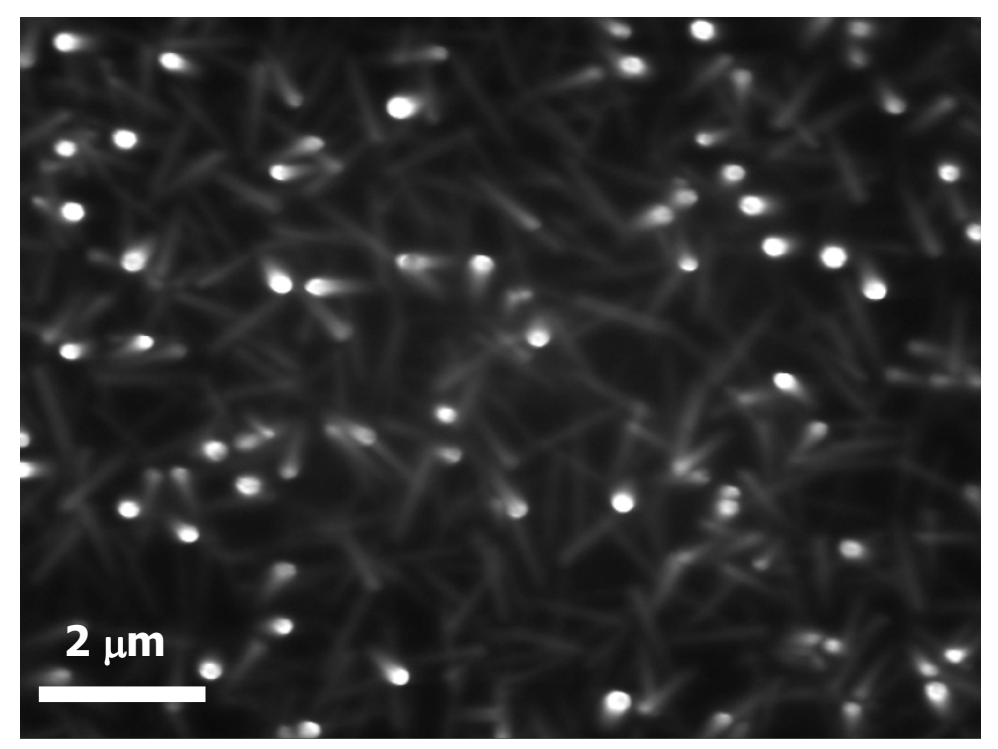

Fig. 9: SEM micrograph of PEDOT:PSS coated PS/ZnO nanostructures

\subsubsection{Deposition of gold contacts}

Using a thermal evaporation method, we applied a gold coating on the top of the PEDOT:PSS layer in order to provide a Schottky barrier type contact for hole injection [170]. The thickness of the gold layer on the top of the sample was approximately $150 \mathrm{~nm}$. The cathodic contacts were applied using flash dry silver paint through a process in which the sample edges were scraped with a knife edge, so as to remove all coatings, and then the silver paint was exposed to those edges. The schematic diagrams of the planar and flexible LED devices are shown in the following Figure 10 (a) and (b). 

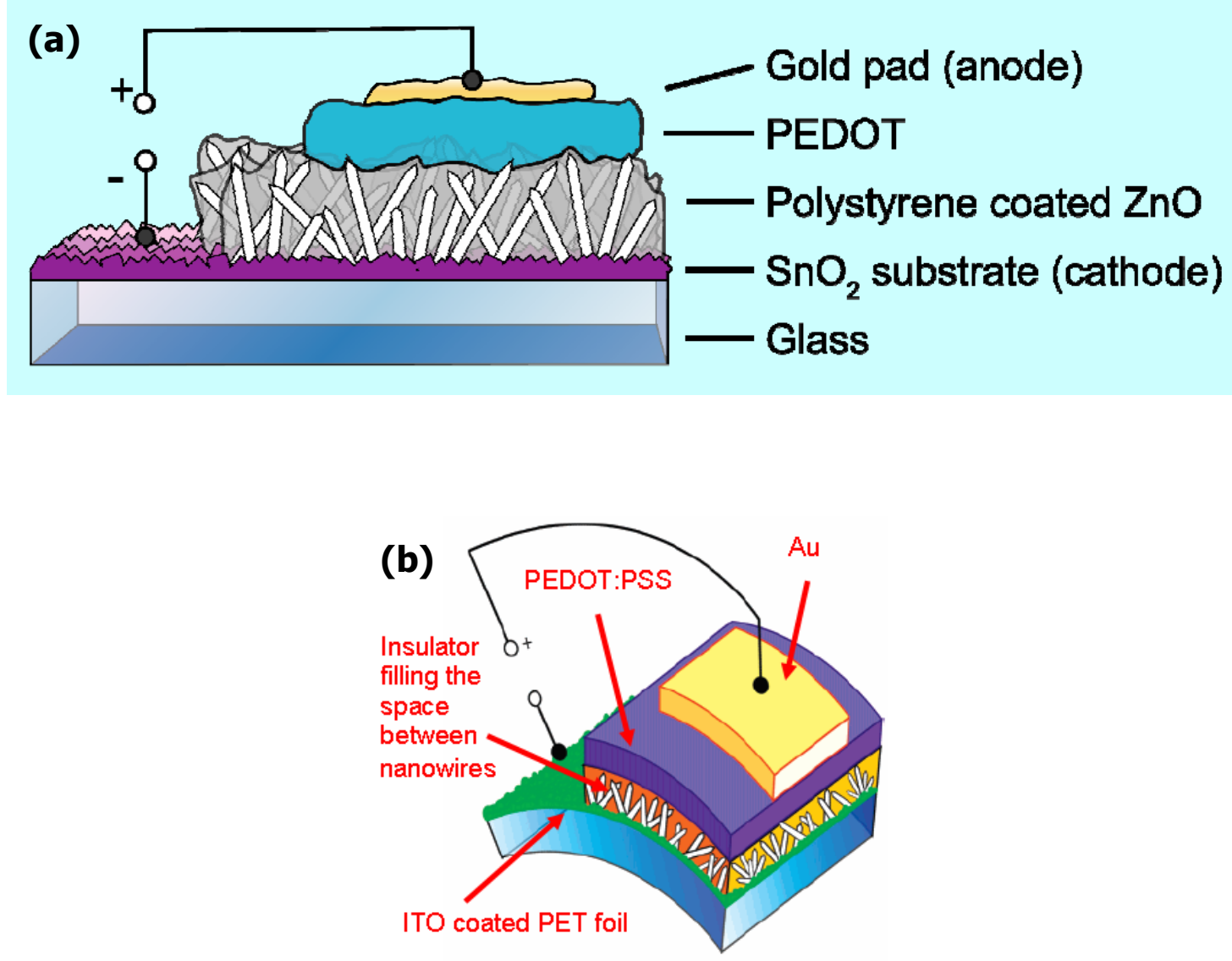

Fig. 10: Schematic diagrams of $\mathrm{ZnO}$ nanowire-based LED arrangements on (a) planar and (b) flexible substrates

\subsubsection{Current-voltage characteristics}

The LED fabricated devices were tested using a HP 4145B semiconductor parameter analyzer in diode channel definition mode. In this mode, source-measure unit 3 and 1 (SMU3 \& SMU1) act as the ground and the positive terminals during forward bias conditions, respectively. In forward bias condition, the ground terminal (cathode) 
was in contact with $\mathrm{SnO}_{2}: \mathrm{F}$ via a silver pad, and the positive terminal was in contact with a gold pad. Reverse bias was achieved by reversing polarity in the applied voltage via the semiconductor analyzer. The HP Semiconductor Analyzer permits the potential to sweep from $-40 \mathrm{~V}$ to $40 \mathrm{~V}$ as well as the reverse. While a function of voltage is applied to the LED, the Analyzer measures the current, allowing a controllable compliance current up to $50 \mathrm{~mA}$. The current-voltage data can be manually transferred from the HP Semiconductor Analyzer to a data analysis program such as Microcal Origin or Microsoft Excel.

\subsubsection{Spectroscopy}

Electroluminescence spectra were measured using the Ocean Optics USB2000 Miniature Fiber Optic Spectrometer with grating \# 13, a grating which has groove densities of 300, an optical resolution of $2 \mathrm{~nm}$, and is optimized for low light conditions in the 300 to $1100 \mathrm{~nm}$ range. Electroluminescence from the nanowire sample was collected through the substrate using a collection lens with a UV/VIS premium fiber optic cable. Light from the optical fiber entered the USB2000 spectrometer through a $100 \mu \mathrm{m}$ entrance aperture onto grating \# 13. The detector collection lens focuses the tall slit images onto the short detector pixels of the 2048-element linear silicon CCD array. In low light conditions, the detector collection lens causes improvement of light efficiency by about 10 times. Grating \# 13 has a spectral range from 300-2000 nm, and a best efficiency spectral range of $300-1100 \mathrm{~nm}$, which is also the response range of the detector. Figure 11 is a simplified diagram depicting the optical/electrical measurement 
setup including electrical and fiber connections. OOIBase32 software from Ocean Optics was used to observe and record the spectral measurements. The software displayed the intensity (counts) of emitted light as a function of wavelength (nanometers). Integration times of 30 to 60 seconds were used to enhance signal strength. The spectra were measured using scan averages of five, and boxcar averages of five, in order to remove some noise and smooth the plot. In boxcar averages, each data point is averaged with its neighboring points to smooth the plot.

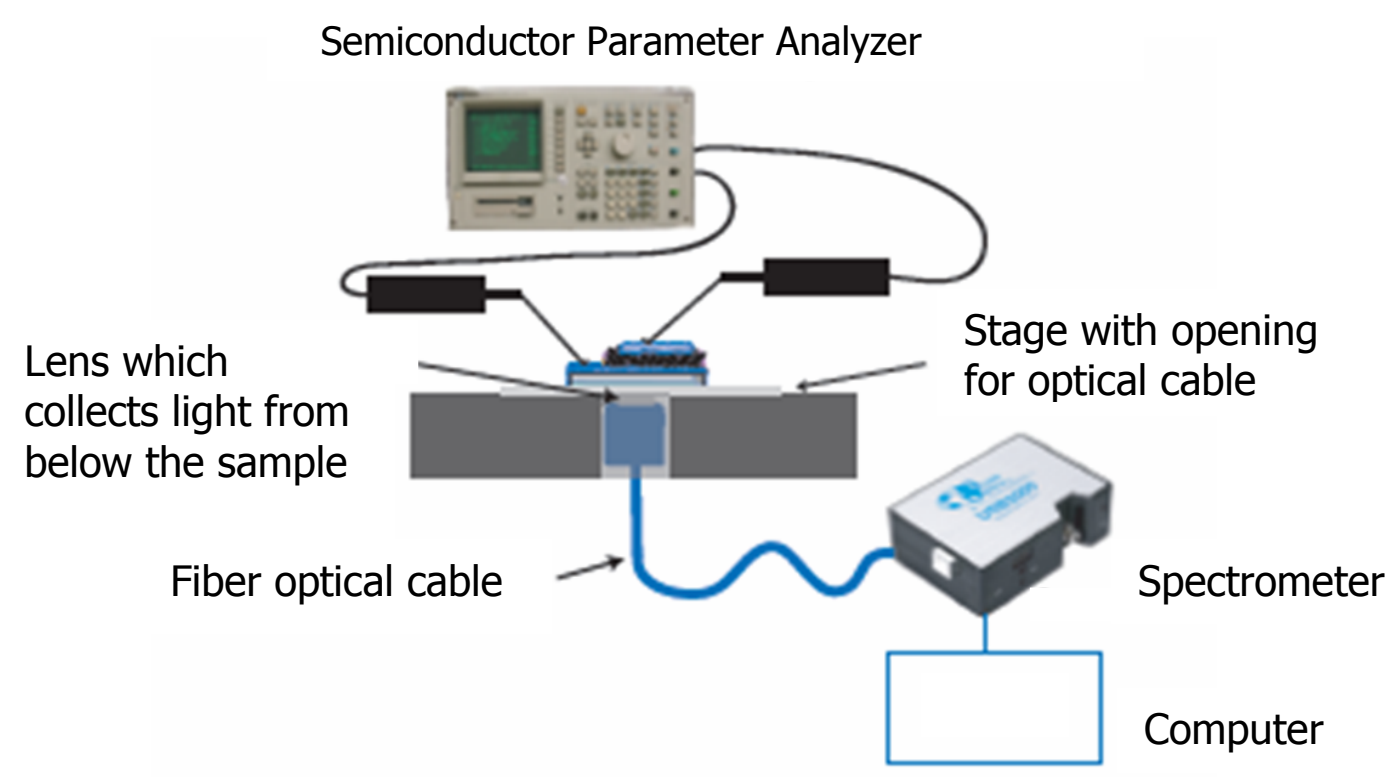

Fig. 11: Schematic diagram of electrical and optical measurements setup 
3.4 Nanowires-quantum-dot-polymer heterojunction solar cells

\subsection{1 $\mathrm{ZnO}$ thin film deposition}

Prior to $\mathrm{ZnO}$ nanowire electrodeposition, the transparent conductive fluorinedoped $\mathrm{SnO}_{2}$ (FTO) coated glass substrates, provided by Pilkington, were covered with a thin continuous layer of $\mathrm{ZnO}$ (see Figure 12) by spray pyrolysis at $300^{\circ} \mathrm{C}$, a similar method to the one performed by O'Regan et al., 2000 [169]. A $0.05 \mathrm{M}$ solution of zinc acetate $\left(\mathrm{Zn}\left(\mathrm{CH}_{3} \mathrm{CO}_{2}\right)_{2}\right)$ in ethanol was made, utilizing a small amount of acetic acid $\left(\mathrm{CH}_{3} \mathrm{COOH}\right)$ to lower the $\mathrm{pH}$. FTO, which we used as a substrate, was placed onto a glass slide that sat on a hot plate in order to distribute the heat evenly. The substrate was heated to $300^{\circ} \mathrm{C}$ before the spraying began. The solution was applied using an airbrush, through which thin films of varying thickness were created by changing the number of sprays, with the optimum thickness requiring 45 sprays. The sprayed substrates were then annealed in air at $380^{\circ} \mathrm{C}$ for two hours. 


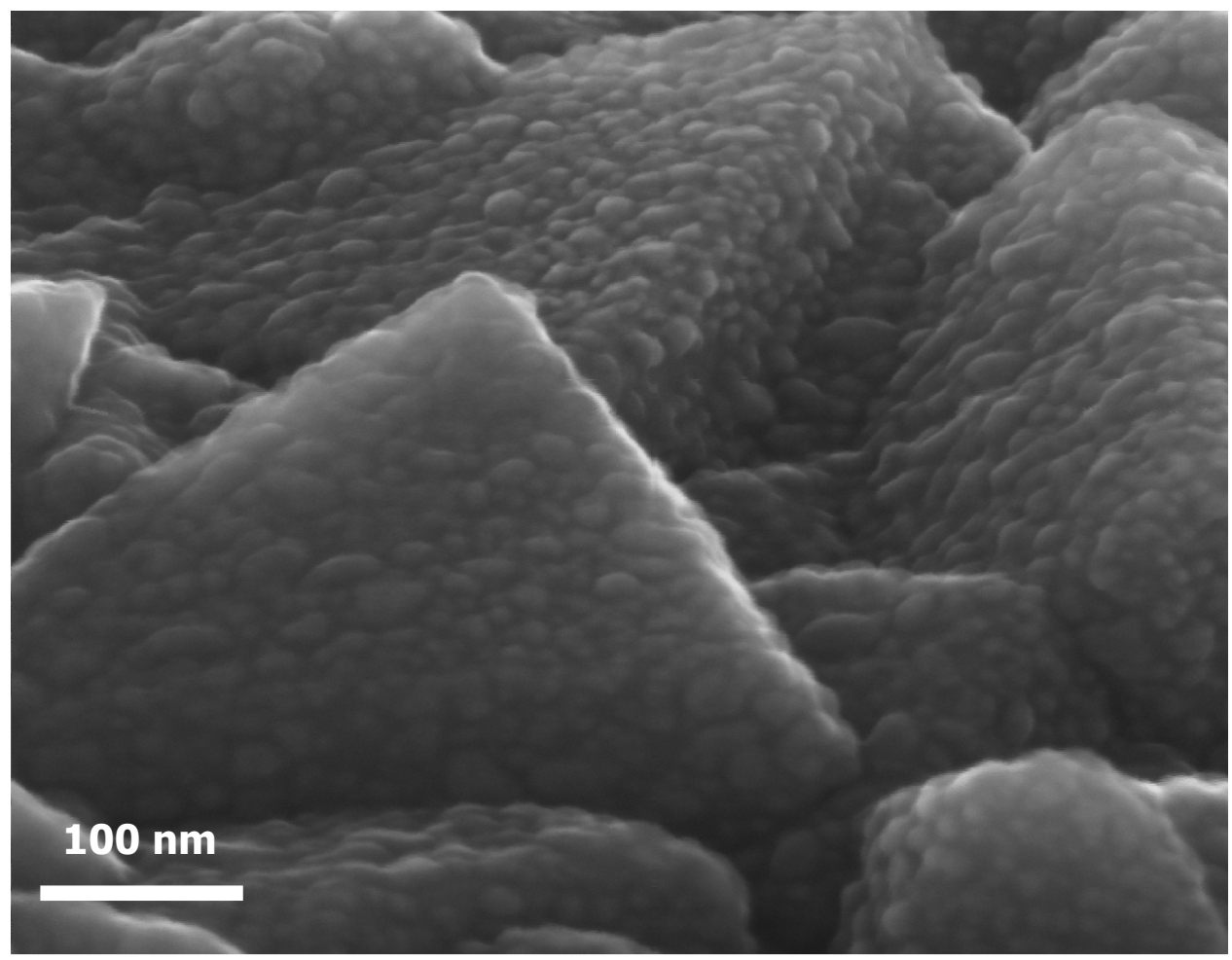

Fig. 12: SEM micrograph of spray-coated $\mathrm{ZnO}$ thin film covered FTO substrate

\subsubsection{Electrodeposition of $\mathrm{ZnO}$ nanowires}

The procedure used for growing $\mathrm{ZnO}$ nanowires is a standard three-electrode electrodeposition process described by previous section 3.1.1. 


\subsubsection{CdSe quantum dots}

\subsubsection{Synthesis of CdSe quantum-dots}

The home-made CdSe quantum dots were synthesized using $\mathrm{CdO}$ and elemental Se as precursors via the procedure described by Boatman et al., 2005 [171]. Following their process, $30 \mathrm{mg}$ of selenium (Se) was combined with $5 \mathrm{~mL}$ of octadecene and 0.4 $\mathrm{mL}$ of trioctylphosphine. This solution was stirred and heated until dissolved and then cooled to room temperature. Simultaneously, cadmium oxide $(\mathrm{CdO})$ was combined with $10 \mathrm{~mL}$ of octadecene and $0.6 \mathrm{~mL}$ of oleic acid. This solution was heated to $225^{\circ} \mathrm{C}$, at which time $1 \mathrm{~mL}$ of the Se solution was added. As the quantum dots grew in size, the solution changed color, starting from its initial yellow color to a dark red or brown (see Figure 13). For absorption and emission spectra analysis, samples were taken whenever a significant color change was noted, approximately $5 \mathrm{sec}$ apart at the start of the reaction and extending as the growth rate decreased. The absorbance spectra were taken with a Beckman DU-7U UV-Vis Spectrophotometer, and the emission spectra were taken using a $355 \mathrm{~nm}$ line of a Nd:YAG laser. As-synthesized quantum dots are capped with long chain length ligand molecules to disable their further growth. These initial ligand molecules obstruct charge transport, producing insulating thin film. In order to create conductive quantum-dot films, we used a solution-phase ligand exchange process to replace long chain ligand molecules with short chain ligand molecules. This ligand exchange was then carried out by a method that is put forth by Landi et al., 2006 [172]. 


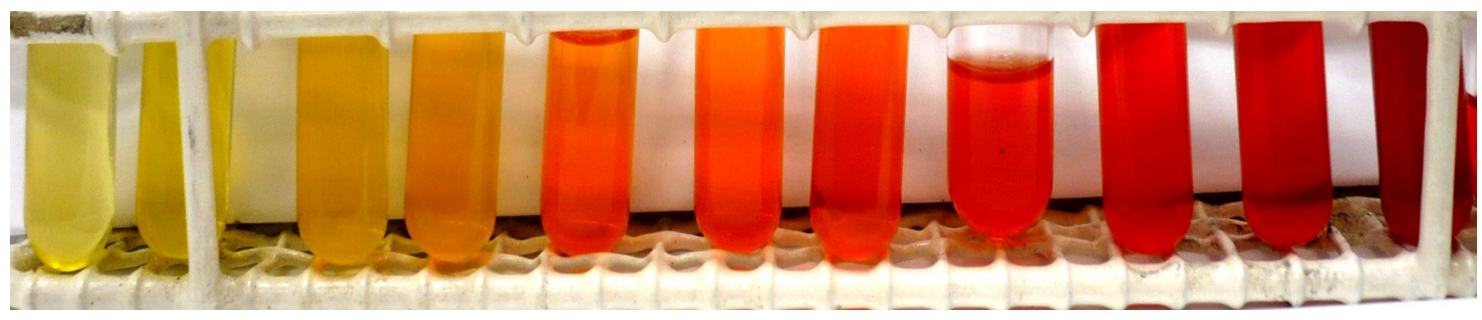

Fig. 13: Digital photograph of various sizes of home-made CdSe quantum dots

\subsubsection{CdSe quantum dot deposition}

\subsection{Drop-coating of CdSe quantum-dots}

The CdSe quantum dots used to create our nanostructured solar cells were either homemade or synthesized quantum dots purchased from NN-Labs. The NN-Labs CdSe quantum dots have an average size of $7 \mathrm{~nm}$, a concentration of $2 \mathrm{~g} / \mathrm{L}$ suspended in toluene, and are capped with long chain octadecylamine ligand molecules. In the earlier stages of our solar cell work, nanowire-quantum-dot based solar devices were prepared by a drop-coating of $\mathrm{CdSe}$ quantum dots on $\mathrm{ZnO}$ nanowire film at an elevated temperature ranging from $20^{\circ}$ to $70^{\circ} \mathrm{C}$. Prior to CdSe deposition, numerous postdeposition methods were explored in an attempt to optimize the homogeneity of the CdSe quantum dot coatings. First, $\mathrm{ZnO}$ nanowire samples were annealed at $380^{\circ} \mathrm{C}$ in ambient/air for two hours. Second, the surfaces of the $\mathrm{ZnO}$ nanowires were cleaned with oxygen plasma for several minutes [173]. Third, the drop-coating of these CdSe quantum dots was carried out at an elevated temperature with several sequences of depositions, where each successive drop coating occurred at a 10 minute interval from 
the most previous deposition for the total evaporation of solvent. The best way of achieving conformal quantum dot film using this method was to first oxygen-plasma clean the $\mathrm{ZnO}$ nanowires for $10 \mathrm{~min}$ and then drop-coat the $\mathrm{CdSe}$ suspension onto the nanowires while they were heated to $40^{\circ} \mathrm{C}[174]$.

\subsection{Layer-by-layer dip-coating of CdSe quantum-dots}

In order to create a crack-free quantum dot film on our $\mathrm{ZnO}$ nanowire sample, we chemically treated CdSe quantum dots with 1,2-ethanedithiol (EDT) ligand and pyridine solvent. Such solution and ligand exchanges are found to be the best methods for obtaining a homogeneous $\mathrm{CdSe}$ quantum dot layer on $\mathrm{ZnO}$ nanowires. The solution exchange process entails the replacement of the initial toluene solution with a pyridine solution using a centrifuge technique. In this case, $1 \mathrm{ml}$ of CdSe quantum dots suspended in toluene were precipitated by adding $5 \mathrm{ml}$ of methanol, and this mix was then centrifuged for 7 minutes at a spin rate of $9000 \mathrm{rpm}$. After centrifugation, the supernatant was discarded, and the precipitant was cleaned with methanol and then dried with $\mathrm{N}_{2}$ gas. Subsequently, these cleaned-quantum dots were dissolved in $2 \mathrm{ml}$ of pyridine solution, and were then sonicated for 5 minutes in order to achieve a homogeneous dispersion of quantum dots in the pyridine solution for further use. Then, the quantum dots suspended in pyridine were deposited on $\mathrm{ZnO}$ nanowire film by a layer-by-layer dip-coating technique, which utilizes 1,2-ethanedithiol (EDT) as a binding agent to remove the initial electrically insulating capping ligand molecules and to enhance the electronic coupling of the quantum dots [175]. Our layer-by-layer dip- 
coating method consisted of dipping the $\mathrm{ZnO}$ sample in a $1 \mathrm{~g} / \mathrm{L}$ concentration of $\mathrm{CdSe}$ quantum dot solution for $30 \mathrm{~S}$, slowly removing the $\mathrm{ZnO}$ sample from the quantum dot solution, drying the sample, dipping it into a beaker containing $0.1 \mathrm{M}$ EDT in anhydrous acetonitrile for $10 \mathrm{~min}$, quickly removing it from EDT solution, and then rinsing the sample in acetonitrile. By repeating this two-step dip-coating procedure, the desired thickness of CdSe quantum dot film was achieved on our $\mathrm{ZnO}$ nanowires.

\subsubsection{3 $\mathrm{CdCl}_{2}$-aided thermal annealing of $\mathrm{CdSe}$ quantum dot film}

The as-deposited CdSe quantum dot film was subsequently annealed in air at $380^{\circ} \mathrm{C}$ in the presence of $\mathrm{CdCl}_{2}$ crystals. Vapors from $\mathrm{CdCl}_{2}$ are often used to slightly p-dope CdTe and to improve the crystallinity of CdTe films, therefore we incorporated this $\mathrm{CdCl}_{2}$ treatment for that same purpose [176]. We then tested whether the use of either, a vacuum ambient to prevent oxidation, or, annealing in air to allow better transmission of the $\mathrm{CdCl}_{2}$ vapors to the sample surface, would produce optimal annealing conditions. It was concluded that annealing in air, in the presence of $\mathrm{CdCl}_{2}$, produced the best performance from the solar cell. In addition, the duration of the anneal was varied successively from 2 hours to 24 hours in two hours increments with the oven initially set to $380^{\circ} \mathrm{C}$ and gradually decreasing to room temperature. 


\subsubsection{Hole conducting coatings}

To create a p-n junction, we applied a hole-conducting layer of MEH-PPV, P3HT, or CuSCN by spin- or drop-coating. Depending on the specific p-type layer, annealing temperatures ranged from $100^{\circ} \mathrm{C}$ to $200^{\circ} \mathrm{C}$, in a vacuum, for a few minutes to several hours. These p-type materials were dissolved in organic solvents and spin- or drop-coated to form a quantum dot film sandwich between a p-n junction of the hole conductive layer and n-type $\mathrm{ZnO}$ nanowire film. Organic polymer layers were deposited by spin coating at $1500 \mathrm{rpm}$ for $60 \mathrm{~s}$. The CuSCN layers were applied by drop coating from a saturated solution of CuSCN in propyl sulfide at elevated temperature of $80^{\circ} \mathrm{C}$.

\subsubsection{MEH-PVV coating}

\section{Poly[2-methoxy-5-(2-ethylhexyloxy)-1,4-phenylenevinylene] (MEH-PPV) is a} p-type light-emitting conjugated polymer used in organic light emitting diode applications. The MEH-PPV, with an average molecular weight of 150,000-250,000 amu, and a peak absorption of $\lambda_{\max }=498 \mathrm{~nm}$, was purchased from Sigma-Aldrich.

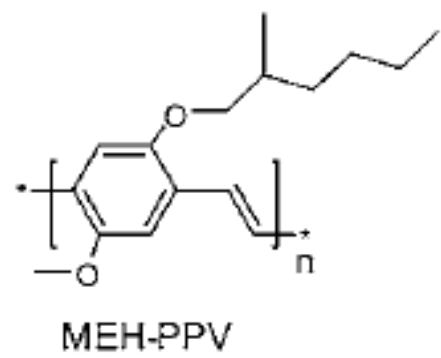

Fig. 14: Chemical Structure of MEH-PPV $\left\{\left(\mathrm{C}_{18} \mathrm{H}_{28} \mathrm{O}_{2}\right)_{\mathrm{n}}\right\}$ 
This polymer has a hole mobility in the range of $10^{-6}$ and $10^{-7} \mathrm{~cm}^{2} / \mathrm{Vs}$ at roomtemperature [177-180]. The highest occupied molecular orbital (HOMO) levels were found to be in the range of 5.1 and $5.2 \mathrm{eV}$, and the lowest unoccupied molecular orbital (LUMO) levels varied from $2.7-3.1 \mathrm{eV}$, resulting in band gap values between 2.1-2.4 $\mathrm{eV}[181-185]$

The MEH-PPV powder was dissolved in chloroform at concentrations varying from $2 \mathrm{~g} / \mathrm{L}$ to $5 \mathrm{~g} / \mathrm{L}$ to obtain a dark red colored solution. We mixed the solution in varying stages, usually adding $1 \mathrm{~g} / \mathrm{L}$ at a time, and allowing that quantity to dissolve before increasing the concentration so to avoid agglomeration. In order to prevent inhomogeneous deposition of MEH-PPV, the solution was spread over the sample and then spin coating was immediately initiated. Lower concentrations of MEH-PPV resulted in more homogeneous depositions, due to the reduced viscosity of the solution. Depending on the CdSe quantum dot layer thickness, the number of MEH-PPV coatings was varied from one to six layers of $5 \mathrm{~g} / \mathrm{L}$ on the $\mathrm{CdSe} / \mathrm{ZnO}$ nanostructure, providing enough coverage to prevent the direct contact of gold on the CdSe film. The sample was then annealed in vacuum ( 1 torr) at a temperature of $125^{\circ} \mathrm{C}$ for 5 hours, and subsequently allowed to cool to room temperature in vacuum. Annealing at this temperature enhances the solar cell's performance by improving the structural order of the MEH-PPV, and increasing the mobility of the charge carriers [177]. 


\subsubsection{P3HT coating}

Poly (3-hexylthiophene-2,5-diyl) (P3HT) is a p-channel organic semiconductor, which we used instead of MEH-PPV as a p-type layer for our recent solar cells. The P3HT layer was incorporated by spin-coating, providing a much better hole mobility in the range of $10^{-3}$ and $10^{-4} \mathrm{~cm}^{2} / \mathrm{Vs}$, at room-temperature, as compared to MEH-PPV, and thereby assisting in an excellent hole transport $[186,187]$. This material was also purchased from Sigma Aldrich and has a molecular weight in the range of 30,000$60,000 \mathrm{amu}$.

There is some disagreement in the literature available on P3HT concerning its bandgap values. The band gap of P3HT is $2.0 \mathrm{eV}$ with a highest occupied molecular orbital (HOMO) level of $5.2 \mathrm{eV}$ and a lowest unoccupied molecular orbital (LUMO) level of $3.2 \mathrm{eV}[188]$.

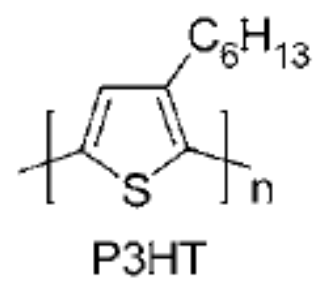

Fig. 15: Chemical Structure of P3HT $\left\{\left(\mathrm{C}_{10} \mathrm{H}_{14} \mathrm{~S}\right)_{\mathrm{n}}\right\}$

P3HT was dissolved in chloroform at concentrations varying from $3 \mathrm{~g} / \mathrm{L}$ to 10 g/ L. The lower concentration was used to obtain a thin layer, so that the majority of 
photo-generated carriers would be within the diffusion length of the interface between the P3HT and the CdSe quantum dot film. The higher concentration, however, was investigated to determine if the thicker layer of P3HT would form a better p-n junction with the $\mathrm{CdSe} / \mathrm{ZnO}$ nanostructure and therefore result in better rectification in the I-V curves. Our method of post deposition was to anneal the P3HT coated samples in vacuum ( 1 torr) at a temperature of $200^{\circ} \mathrm{C}$ for $20 \mathrm{~min}$, and then to allow the samples to gradually cool back down to room temperature in vacuum.

\subsubsection{CuSCN deposition}

In order to make totally inorganic solar cells, we used copper (I) thiocyanate $(\mathrm{CuSCN})$ as a hole transport layer instead of p-type polymers, as we used in the earliest cases. The hole mobility in $\mathrm{CuSCN}$ has been reported to be approximately 10-20 $\mathrm{cm}^{2} / \mathrm{Vs}$ [189]. It is a p-type semiconductor with a wide band gap of $\sim 3.6 \mathrm{eV}$, making it transparent to visible light. To create a saturated CuSCN solution, the amount of $\mathrm{CuSCN}$ added is slightly greater than the amount soluble and thus leaves a powder on the bottom of the vessel. In our case, $0.2 \mathrm{~g}$ of CuSCN was dissolved in $10 \mathrm{~mL}$ of $\mathrm{n}$ propylsulfide $\left(\mathrm{CH}_{3} \mathrm{CH}_{2} \mathrm{CH}_{2}\right)_{2} \mathrm{~S}$ [190]. Since the CuSCN dissolves slowly in propylsulfide, the mixture was sonicated for a few hours and then allowed to settle for at least one additional day. Prior to deposition, $2 \mathrm{~mL}$ of CuSCN solution was taken from the saturated solution and diluted with $250 \mu \mathrm{L}$ of propylsulfide. The sample was then placed on a hot plate at $80^{\circ} \mathrm{C}$, the solution was lightly spread on the surface using a 
syringe, and blown with nitrogen to allow the sample to dry. Both the CuSCN and propylsulfide were purchased from Sigma Aldrich.

\subsubsection{Back contact deposition}

The HOMO level of the p-type material must match the metal work function in order to choose a back contact [182]. Therefore, we used gold as a back contact for all of our devices because the HOMO level of the p-type and the work function of the $\mathrm{Au}$ are approximately equal. The gold contact was deposited with a thermal vacuum evaporator that consists of a vacuum chamber with a tungsten basket that can be heated to evaporate gold wire placed within it.

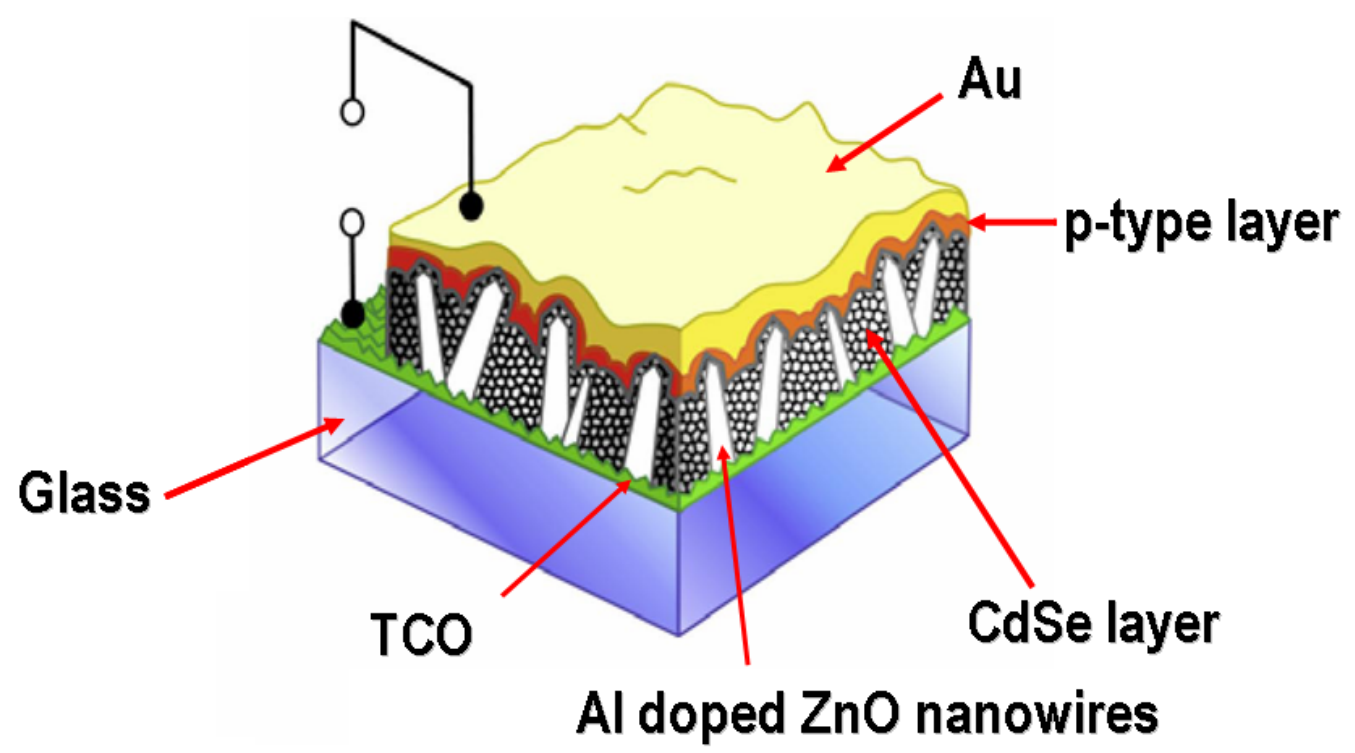

Fig. 16: Schematic diagram of nanowire-quantum-dot solar cell 


\subsubsection{Current-voltage measurements}

A Keithley 236 Source Measure Unit (SMU) was used to characterize the current-voltage measurements of our solar cell under light and dark conditions. SMU is widely used for characterizing diodes and transistors, and it is capable of applying a known voltage and measuring the resulting current or vice versa. In our case, the probe applying the voltage contacted to the FTO substrate via a silver pad while the probe measuring the current contacted to the gold pad. A voltage sweep was applied from -1.0 $\mathrm{V}$ to $1.0 \mathrm{~V}$ with a voltage step of $0.02 \mathrm{~V}$, a compliance of $100 \mathrm{~mA}$, and a delay time of 3s. Photoconversion of the solar cells was characterized by I-V curves in the dark and under quartz-halogen lamp illumination. The sample was illuminated through the FTO side. The dark measurements were made under a black box completely covering the optical bench. The intensity of the white light was adjusted such that in the absorption region of the solar cell, $350 \mathrm{~nm}<\lambda<780 \mathrm{~nm}$, the spectrum had the same number of photons as the solar AM1.5 spectrum at $85 \mathrm{~mW} / \mathrm{cm}^{2}$. The pad size of the Au contacts was $0.077 \mathrm{~cm}^{2}$. 


\subsubsection{Photoresponse measurements}

The photoresponses of the solar cells were measured at short-circuit conditions using a Sciencetech 9055 Monochromator, a chopper wheel, and a white light source. Using this system, white light entering the monochromator reflects onto the diffraction grating, as the grating rotates a different wavelength can be selected through the exit slit. The input of the fiber optic cable is attached to the exit slit, and the output is attached beneath the measurement stage. The typical wavelength range of our solar cell measurement was 300 to $800 \mathrm{~nm}$.

The sample's spectral response was measured with an EG\&G 5208 Two Phase Lock-in Analyzer. During the process described above, light incident on the entrance slit of the monochromator must first pass through a chopper which operates at a specific frequency. The lock-in amplifier filters out noise and interference in order to detect only the signal with the same frequency as the one generated by the chopper. The measured current is therefore the photocurrent contribution without any dark current contribution.

A Keithley 428 Current Amplifier was connected to the lock-in amplifier in order to amplify the photogenerated signal. This signal is then detected by the lock-in analyzer. The spectral response can also be measured for a range of applied voltages by using the current amplifier to supply a voltage to the probes in contact with the sample. The photocurrent can then be calculated from the lock-in analyzer data. 


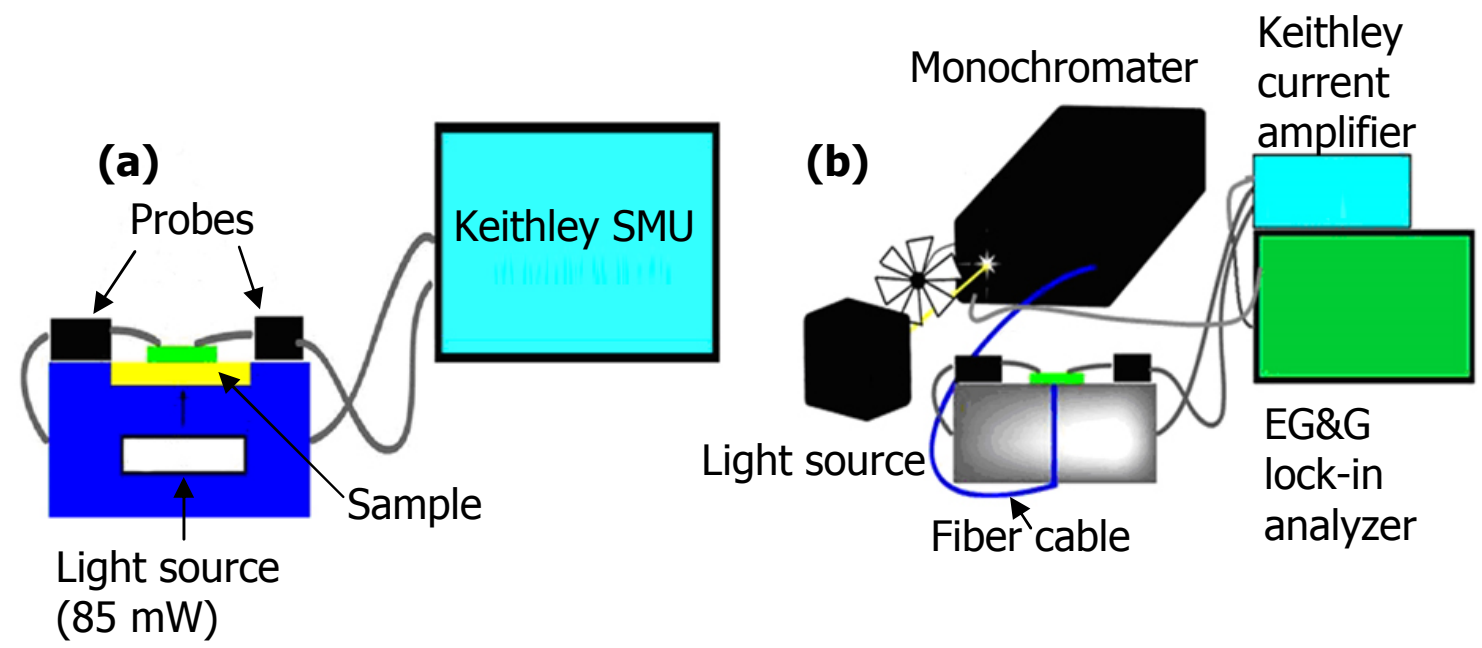

Fig. 17: Schematic diagram of (a) current-voltage (IV) and (b) photoresponse measurement setups

The intensity of the monochromator output was calculated by comparing the spectral response of a calibrated photodiode to the spectral response output as measured by the lock-in analyzer. The responsivity of the photodiode was provided in the photodiode specification information. The intensity of the monochromator is given by the following equation:

$$
\text { Intensity }(\lambda)=\text { Si Photodiode Photocurrent }(\lambda) / \text { Responsivity }(\lambda)
$$

The energy of the incident photons was calculated as follows:

$$
E_{\text {photon }}=h \mathrm{c} / \lambda_{\text {photon }}
$$


where, $h$ is Planck's constant, $\mathrm{c}$ is the speed of light, and $\lambda$ is the wavelength of the incident photon.

The number of incident photons per second is given by:

$$
\text { Number of Photons }(\lambda) / \text { second }=\text { Intensity }(\lambda) / E_{\text {photon }}(\lambda)
$$

The gain that was applied to obtain the photocurrent is given by:

$$
\text { Photocurrent }(\mathrm{A})=\mathrm{EG} \& \mathrm{G} \text { voltage reading/Applied Gain }
$$

Then the number of electron/hole pairs generated per second is given by:

$$
\text { Electrons/ second }=\text { Photocurrent/charge of an electron }
$$

The external quantum efficiency is given by:

Quantum Efficiency $(\%)=($ Collected electron/hole pairs per second/ Incident photons per second) x 100 


\section{Results and Discussion}

4.1 Electrochemical growth of $\mathrm{ZnO}$ nanowires and their fundamental properties

\subsubsection{Overview}

In this section, we describe the fundamental properties of our $\mathrm{ZnO}$ nanowires with the intention of establishing a foundational understanding of the material used in the design and optimization of our devices. This basic study demonstrates that these $\mathrm{ZnO}$ nanowires have a single crystalline structure with a hexagonal lattice. We further show that doping of these nanowires from the solution phase in electrodeposition is possible. Finally, we demonstrate that the as-grown nanowires are defect-rich, and that annealing at moderate temperatures can generate marked structural and electronic improvements.

4.1.2 Morphology and structural properties of as-grown $\mathrm{ZnO}$ nanowires

\subsubsection{Appearance of $\mathrm{ZnO}$ nanowires}

Figure 18 (a) shows a scanning electron micrograph of the $\mathrm{ZnO}$ nanowires on a transparent, fluorinated $\mathrm{SnO}_{2}$ /glass substrate, in the as-grown state, after having been grown using the standard $80^{\circ} \mathrm{C}$ electrodeposition process $[65,66,68]$ described in section 3.1.1.1. The nanowires are free-standing single-crystallites with hexagonal 
cross-sections and blunt, slightly rounded or irregular tips. After an hour of deposition, these nanowires typically grow to a height of $\sim 1500 \mathrm{~nm}$, with diameters between 150 $250 \mathrm{~nm}$, and an aspect ratio (length/diameter) of $\sim 10$. The appearance of these nanowires is not changed by the incorporation of impurities with concentrations of 5 $\mu \mathrm{M}$ and below [84]. However, when large amounts of impurities are introduced into the electrolyte the appearance can change noticeably. Figure 18 (b) shows a micrograph of wires obtained under standard conditions, but with a concentration of $20 \mu \mathrm{M} \mathrm{AlCl} \mathrm{Al}_{3}$ added to the solution. Larger $\mathrm{ZnO}$ nanowires and thin zinc hydroxychloride platelets (see arrow) are apparent due to this high concentration of $\mathrm{AlCl}_{3}$.
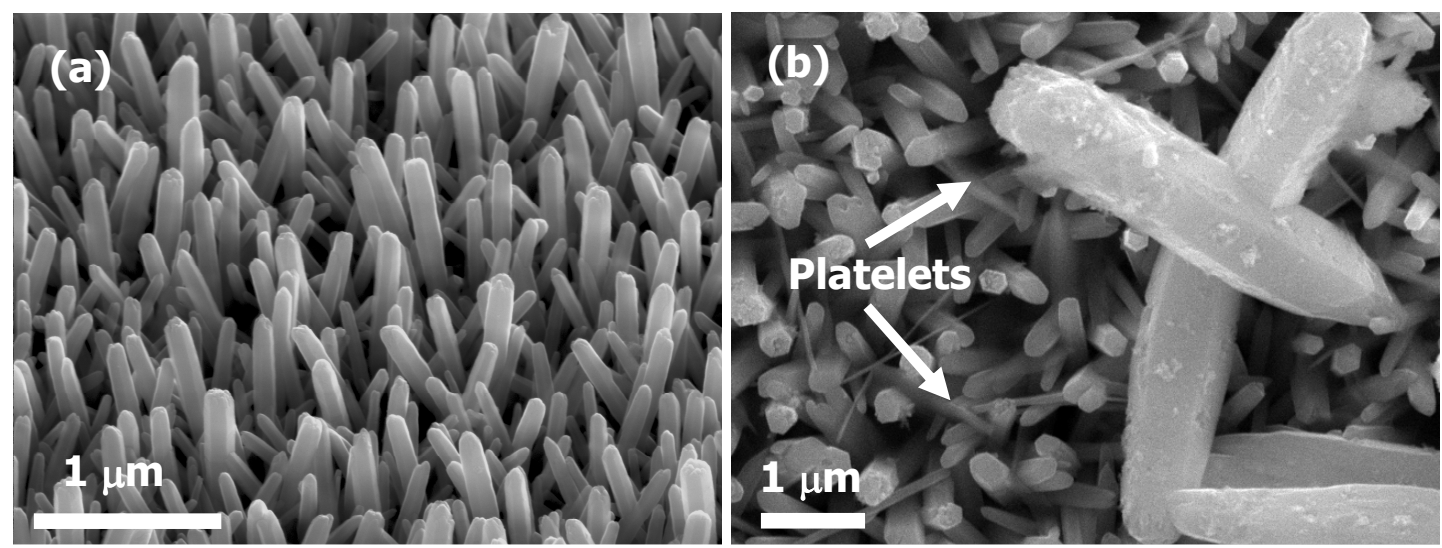

Fig. 18: SEM micrographs of free standing (a) $5 \mu \mathrm{M} \mathrm{Al}$ doped and (b) $20 \mu \mathrm{M} \mathrm{Al}$ doped $\mathrm{ZnO}$ nanowires on an FTO substrate 
4.1.2.2 As-grown $\mathrm{ZnO}$ nanowires: crystal structure and defects

The electrodeposited $\mathrm{ZnO}$ nanowires demonstrate a single crystalline nature, as evidenced by both HRTEM and electron diffraction experiments on single nanowires in Figure 19 (a) and (b).
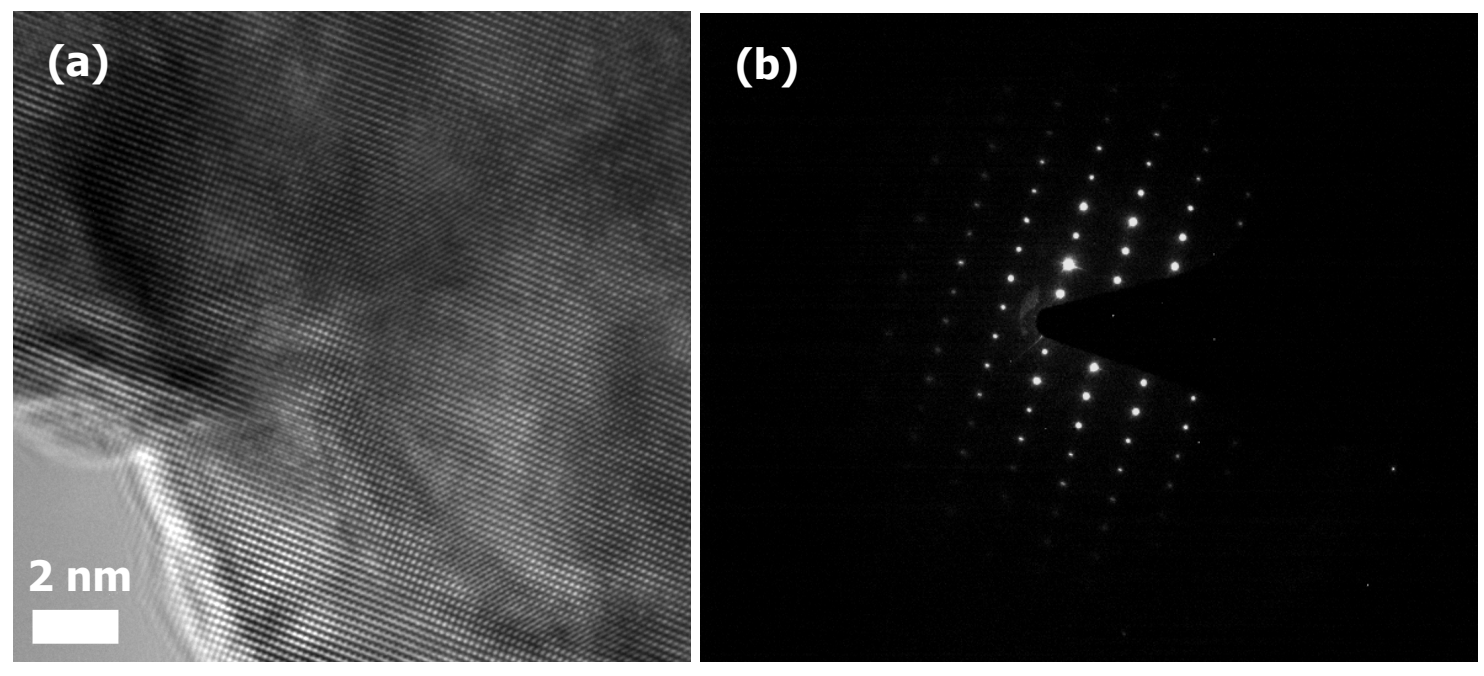

Fig. 19: (a) HRTEM image and (b) selected area electron diffraction pattern of an asgrown $\mathrm{ZnO}$ nanowire

It appears that these as-grown nanowires grown from low-temperature electrochemical method are defect-rich [83]. The obtained TEM dark field image (See Figure 20) from as-grown sample indicates that the strong brightness changes throughout the bulk and surface regions are due to the presence of an excessive strain, crystal lattice defects, and distortions. 


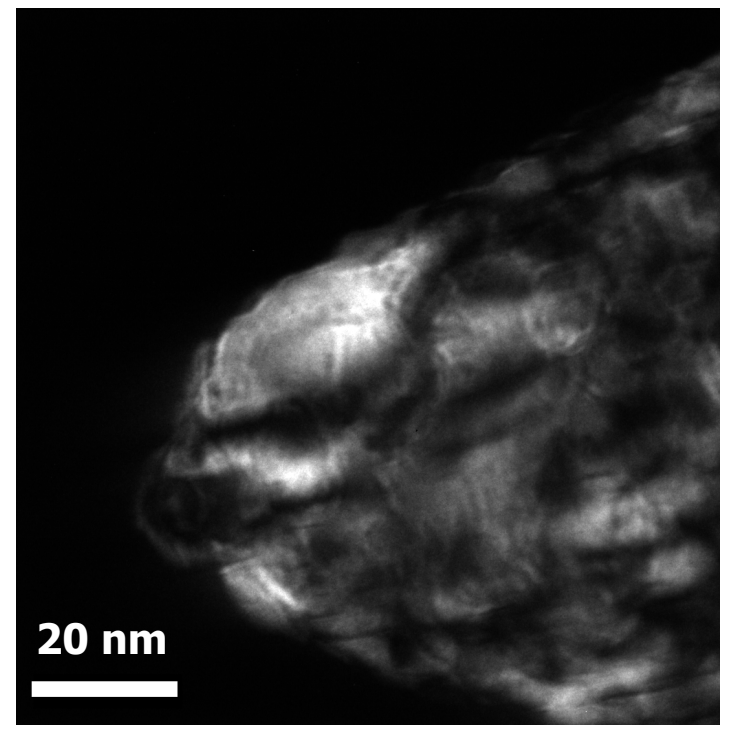

Fig. 20: Dark-field TEM micrographs of a $\mathrm{ZnO}$ nanowire in the as-grown state

4.1.3 Annealed $\mathrm{ZnO}$ nanowires: disappearance of strain defects at $\mathrm{T} \sim 380^{\circ} \mathrm{C}$

Figure 21 (a) presents the HRTEM image of a $\mathrm{ZnO}$ nanowire in the annealed state. Annealing around $380^{\circ} \mathrm{C}$ seemed to improve the crystal structure of $\mathrm{ZnO}$ nanowire by removing strain defects, which arise likely to be in the presence of oxygen vacancies in the as-grown state [83]. The obtained TEM dark field image from annealed $\mathrm{ZnO}$ nanowire also indicates that the annealing process changed the strain defect concentration in the nanowires. This strain relaxation occurs, due to the thermal annealing, at $380^{\circ} \mathrm{C}$, which leads to a more subtle contrast between surface defects, in which the presence of nano-cavities, in Figure 21 (b), are the only visible indications of black and white contrast on the image. However, the high-resolution-TEM images also show a large number of edge dislocations in the annealed state as compared to the as- 
grown state, as is demonstrated in Figures 19 (a) and 21 (a). These HRTEM images suggest that during the annealing process the dislocations move from the bulk towards the surface of the nanowire. Despite the fact that annealing heals defects and releases stress, it also a cause of the conversion of lattice strains into dislocation-type defects with moderate concentrations.
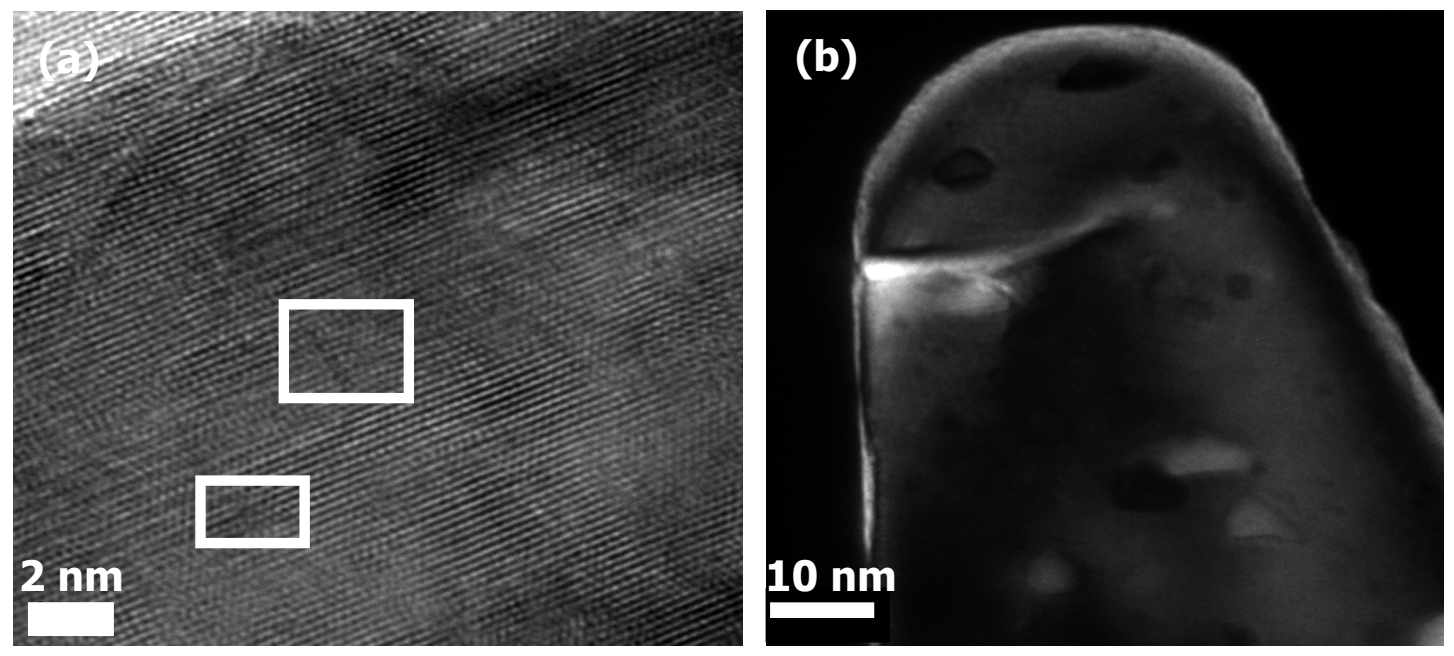

Fig. 21: (a) HRTEM and (b) Dark-field TEM micrographs of a $\mathrm{ZnO}$ nanowire in the annealed state at $380^{\circ} \mathrm{C}$ (the dislocations are marked with white color rectangular boxes)

The selected area electron diffraction (SAD) patterns for the as-grown and the annealed state are shown in Figures 22 (a) \& (b), revealing that the diffraction spots of as-grown nanowires are relatively larger than those of annealed nanowires, due to more diffused intensity associated with local lattice distortions. These results further confirm the idea of a strained lattice in the as-grown state and consequent strain relaxation due 
to the thermal annealing process. Both the electron diffraction patterns and HRTEM images show the single crystalline structure of an electrodeposited $\mathrm{ZnO}$ nanowire before and after annealing, as displayed in Figures 19, 21 (a), \& 22.
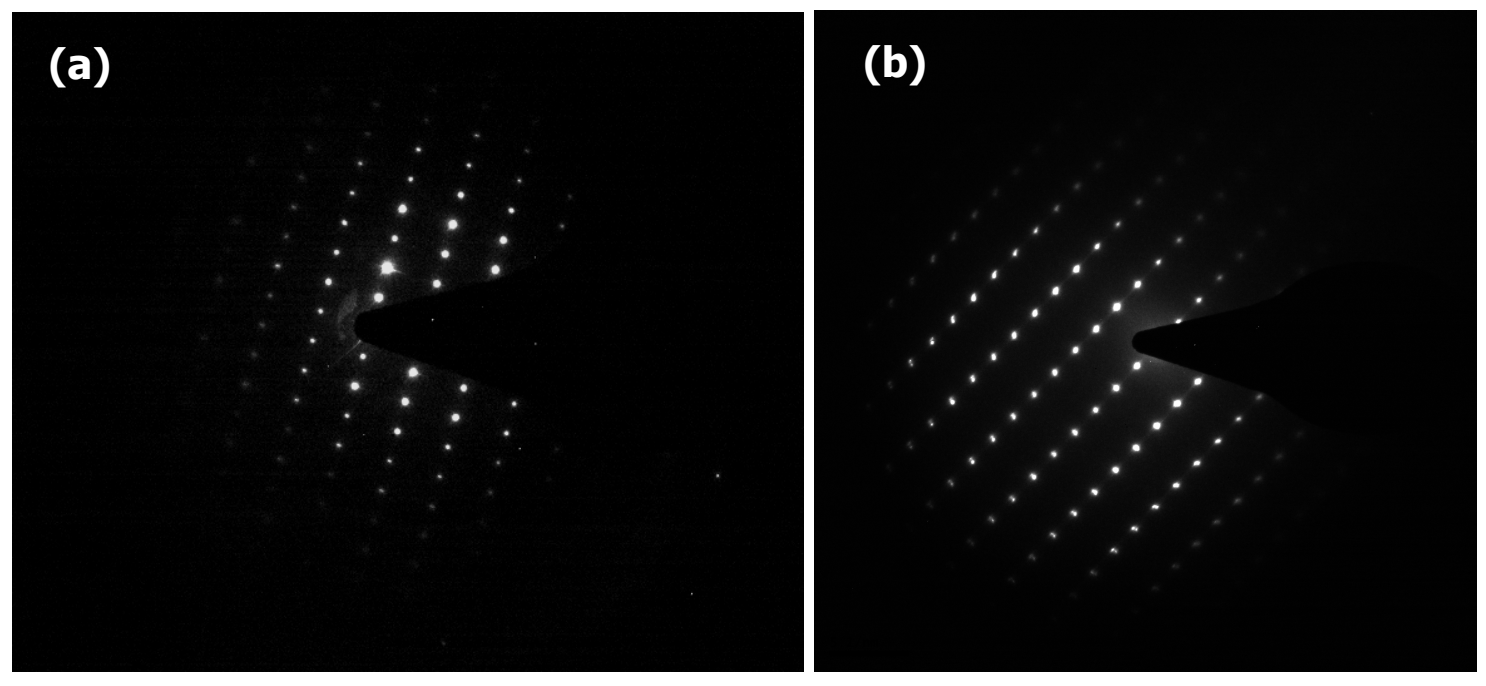

Fig. 22: $\mathrm{SAD}$ patterns for a $\mathrm{ZnO}$ nanowire in the (a) as-grown, and (b) annealed state $380^{\circ} \mathrm{C}$ (the diffraction spot size is reduced in the annealed state)

\subsubsection{Dopant incorporation: SIMS and TEM-EDX measurements}

Energy dispersive X-ray fluorescent (EDX) line profiles and secondary ion mass spectroscopy (SIMS) were used to characterize the local chemical compositions of the nanowires. Figure 23 (a) shows the elemental count rates for $\mathrm{Zn}, \mathrm{O}$, and $\mathrm{Al}$ across the nanowire, as obtained from the STEM-EDX line profile. This line profile was performed across several hundred nanometers of the nanowire, confirming the presence 
of homogeneous doping incorporation in the single nanowire. The count rates for $\mathrm{Al}$, as well as for other doping materials we have tested, closely follow the profile of $\mathrm{Zn}$ and O, indicating that $\mathrm{Al}$ is incorporated in the bulk of the nanowire. The incorporation of doping was also confirmed by SIMS measurements that were carried out using $\left(\mathrm{O}^{2+}\right)$ sputtering analysis ions and $\left(\mathrm{Bi}^{+}\right)$primary analysis ions. The sputtering was performed using an $\mathrm{O}^{2+}$ ion beam, localized to an area of $1 \mu \mathrm{m} \times 1 \mu \mathrm{m}$. Figure 23 (b) shows the $\mathrm{Zn}$, $\mathrm{Al}, \mathrm{Sn}$, and Si signals of the $5 \mu \mathrm{M}$ Al sample on a logarithmic scale versus sputtering time. The $\mathrm{Al}$ count rate seemed to decline proportionally to the $\mathrm{Zn}$ count rate over most of sputtering time. This is a strong indication that $\mathrm{Al}$ is incorporated homogeneously into the $\mathrm{ZnO}$ crystal structure. Similar results have been observed for other doping materials.
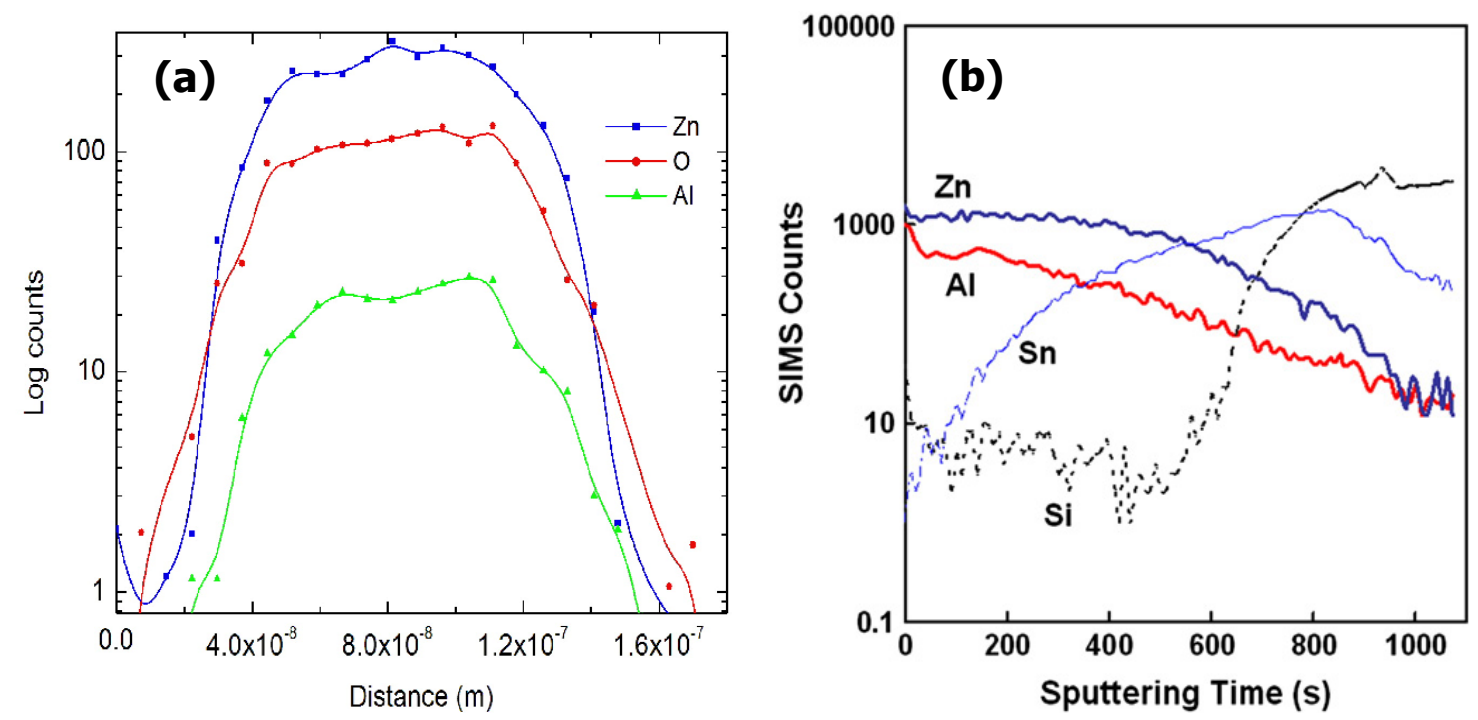

Fig. 23 (a) The STEM-EDX line profile and (b) SIMS sputtering depth profile of a $5 \mu \mathrm{M}$ $\mathrm{Al}$ doped $\mathrm{ZnO}$ nanowire annealed at $380^{\circ} \mathrm{C}$ 
Figure 24 shows a plot of the impurity concentration in the solid as a function of the precursor concentration in the electrolyte. It appears that increasing the dopant concentration in the deposition solution leads to an increase in the incorporation of impurities into the $\mathrm{ZnO}$ nanowires, as displayed in Figure 24. We can also see that the doping concentrations of $\mathrm{Al}, \mathrm{Co}$, and $\mathrm{Cu}$, in the nanowires, are well above $1 \%$ whereas Mn concentrations remain lower than 1\%. These SIMS measurements are in great agreement with the results obtained using the EDX line scan. Furthermore, post growth annealing at $380^{\circ} \mathrm{C}$ did not affect the impurity concentrations in our nanowires, i.e. the out-diffusion of impurities at this moderate temperature is small, as would be expected from the magnitude of typical atomic diffusion coefficients in $\mathrm{ZnO}$.

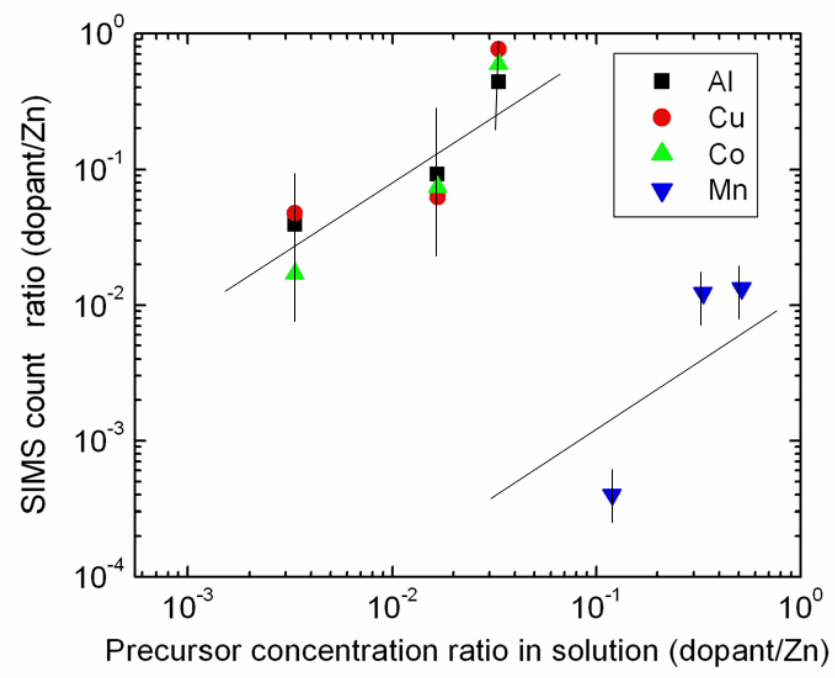

Fig. 24: The SIMS count ratios of the doped $\mathrm{ZnO}$ nanowires as a function of precursor concentrations in the solution 


\subsubsection{Effects of doping: magnetic properties}

Magnetic measurements on undoped and transition metal ion doped $\mathrm{ZnO}$ nanowire films were performed at room-temperature using a vibrating sample magnetometer. Figure 25 (a) shows the magnetic behavior of the different doped samples as a function of applied magnetic field at room temperature. All of the $\mathrm{Co}, \mathrm{Cu}$, \& $\mathrm{Mn}$ doped $\mathrm{ZnO}$ nanowire samples were found to be superparamagnetic at room temperature, while in the $\mathrm{Al}$ doped and undoped $\mathrm{ZnO}$ nanowires there is no magnetic response [85], as shown in Figures 25 (b) \& (c). The typical magnetization strength is in the order of 0.1-0.5 Bohr magneton/impurity. Saturation occurs at $2 \mathrm{kG}$ for Mn and Co doped $\mathrm{ZnO}$ nanowire samples, and at $1 \mathrm{kG}$ for $\mathrm{Cu}$ doped $\mathrm{ZnO}$ nanowire samples. The magnetic curves present in Figure 25 (a) exhibit an S-shaped behavior, however, there is no or only marginal hysteresis. This behavior would be identified as superparamagnetic, which is defined as a ferromagnetic response of fine grains of ferromagnetic materials whose Neel relaxation time is smaller than the typical measurement time. The magnetic results of $\mathrm{Cu}$-doped $\mathrm{ZnO}$ are of particular interest because the secondary phases of $\mathrm{Cu}$ are paramagnetic. Therefore, superparamagnetism in $\mathrm{Cu}$-doped $\mathrm{ZnO}$ is due to the substitution of $\mathrm{Cu}$ ions into the $\mathrm{ZnO}$ lattice sites. This also further confirmed that the successful incorporation of impurity ions into the bulk of the $\mathrm{ZnO}$ nanowires had occurred. Herng et al., 2007 [191] reported a similar conclusion, suggesting that the magnetic response in $\mathrm{Cu}$ doped $\mathrm{ZnO}$ nanowire film could be due to a combination of interstitial $\mathrm{Zn}$ and the substitution of $\mathrm{Cu}$ for $\mathrm{Zn}$ in the $\mathrm{Zn}$ lattice. 

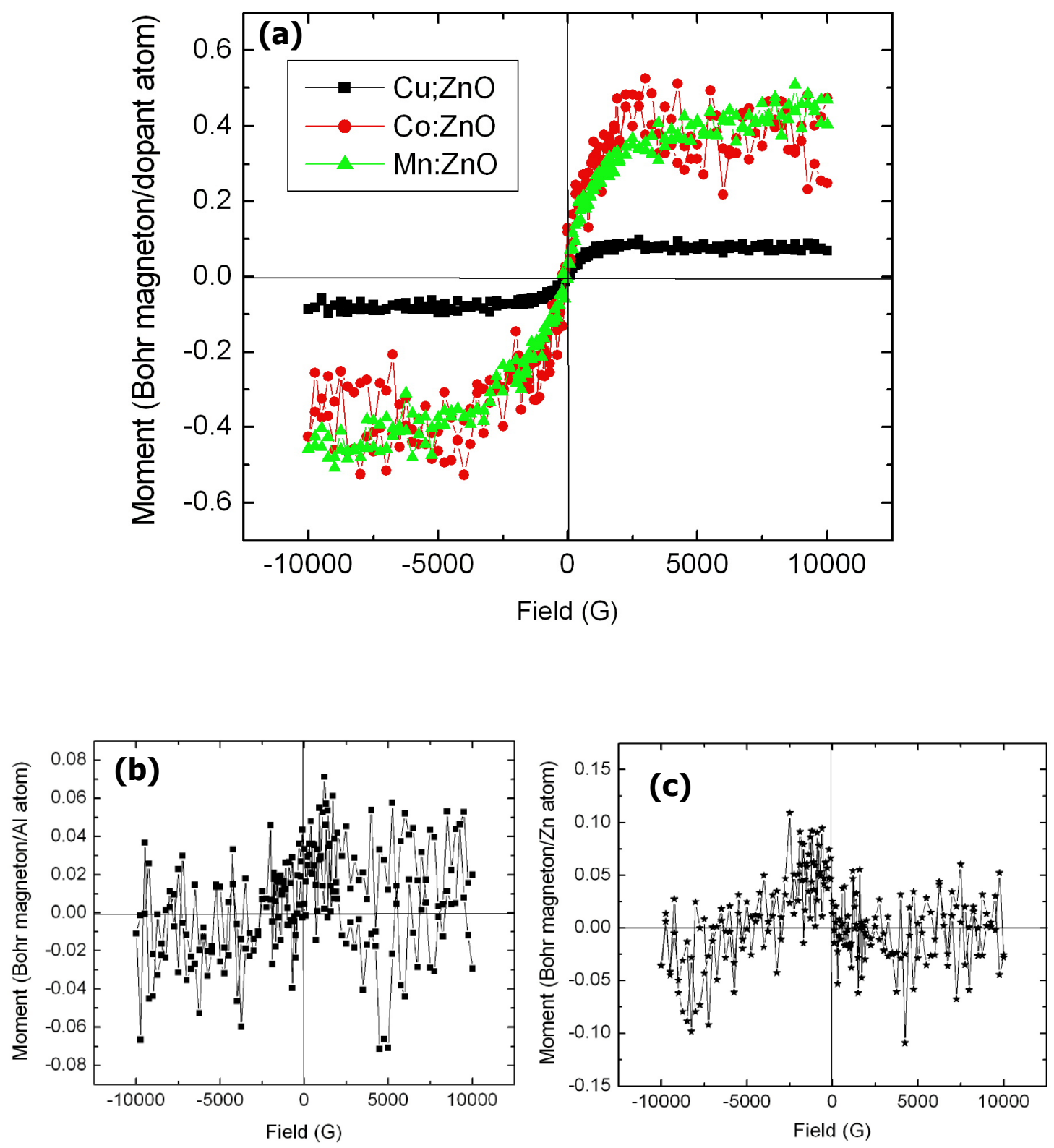

Fig. 25: The room-temperature magnetization curves of (a) various magnetic impurity doped $\mathrm{ZnO}$ nanowires, (b) $\mathrm{Al}$ doped $\mathrm{ZnO}$ nanowires, (c) and undoped $\mathrm{ZnO}$ nanowires, respectively 
4.1.6 Effects of doping: Temperature-dependent photoluminescence measurements

The temperature-dependent photoluminescence (PL) measurements were performed using a $325 \mathrm{~nm} \mathrm{He}-\mathrm{Cd}$ laser line. The comparison of spectra obtained from temperature-dependent photoluminescence (PL) measurements of $\mathrm{ZnO}$ nanowires before and after thermal annealing indicates that the optical activity of impurities changes noticeably upon annealing. Even at room temperature, the photoluminescence obtained from the annealed sample exhibits higher energy excitonic transitions as compared to those in the un-annealed sample, as shown in Figure 26.

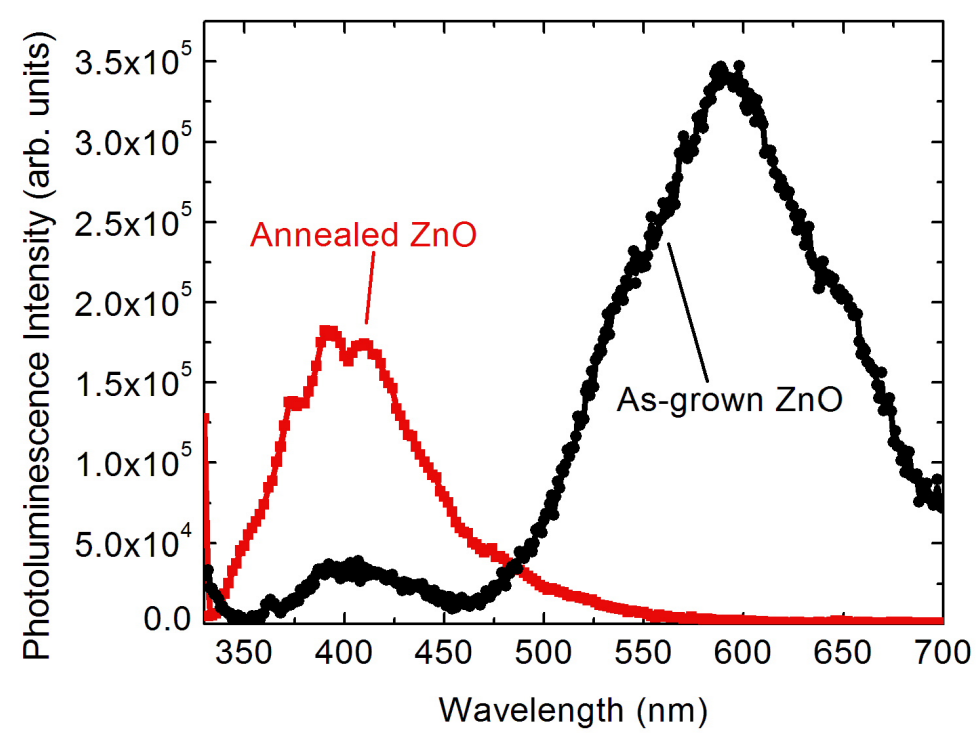

Fig. 26: Room-temperature photoluminescence spectra of $\mathrm{Al}$-doped $\mathrm{ZnO}$ nanowire samples before and after annealing 
The quality of a light-emitting material is determined by internal quantum efficiency, which is defined as the ratio between the number of generated photons and the number of injected carriers. There are several unknown parameters that influence the internal quantum efficiency calculation, such as carrier injection efficiency, a light extraction coefficient, internal absorption, and the volume of excited nanowires, all of which contribute to a complicated measurement. Therefore, in order to quantitatively determine the internal quantum efficiency, we used an all-optical method of detailed comparison with low temperature PL measurement procedures, as reported in references [192, 193]. We can assume that the radiative recombination rate is temperature independent whereas the nonradiative recombination rate is thermally activated. We observed the plateau of a near band-edge emission for Al-doped $\mathrm{ZnO}$ nanowires annealed at $380^{\circ} \mathrm{C}$ at low temperature and high excitation densities. It can be assumed that all of the carriers radiatively recombine at low temperatures, and thus the ratio between the near band-edge emission intensities at room temperature to those at low temperatures is defined as an internal quantum efficiency. Figure 27 illustrates the integrated near band-edge photoluminescence intensity as a function of temperature for Al doped and undoped $\mathrm{ZnO}$ nanowire samples. The internal quantum efficiency for PL was measured to be as high as 16 percent for Al-doped samples annealed at $380^{\circ} \mathrm{C}$. The obtained efficiency of annealed Al-doped $\mathrm{ZnO}$ nanowires is quite comparable with the best reported efficiency to date [193, 194]. 


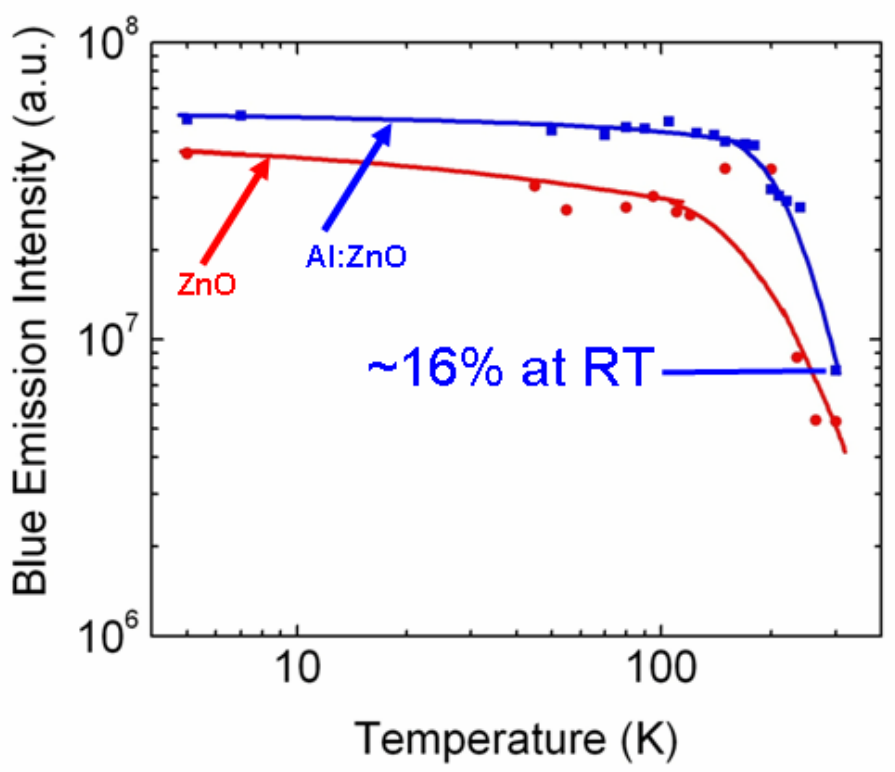

Fig. 27: Integrated intensity of a near band-edge emission at various temperatures for the undoped and $\mathrm{Al}$-doped $\mathrm{ZnO}$ nanowire samples

\subsubsection{Laser annealing: Room-temperature photoluminescence measurements}

The near band-edge room-temperature photoluminescence spectra are obtained from $\mathrm{ZnO}$ nanowires by direct laser annealing at $10 \mathrm{~mJ}$ for various time intervals, as shown in Figure 28. Our findings demonstrated that the near band-edge luminescence was improved by a factor of 8 after 20 min of laser annealing [195]. However, the continuous laser annealing led to a slight reduction in the luminescence efficiency. This degradation is due to the partial melting of the nanowires, as is confirmed from the SEM micrograph, and is shown in Figure 29. The comparison of results obtained from pulse energies varying in the range of $10 \mathrm{~mJ}$ to $40 \mathrm{~mJ}$ is shown in Figure 30 (a). This 
figure reveals that the higher pulse energies lead to faster and more efficient improvement of the luminescence. We can also see that extended laser annealing results in a decrease of the luminescence. The improved luminescence of $\mathrm{ZnO}$ samples laser annealed through $\mathrm{SnO}_{2}$-coated glass and ITO-coated PET foils are shown in Figures 30 (a) \& (b). It appears that the decreasing luminescence of ITO-coated PET foil is due to the peeling of the ITO layer at a pulse energy of $10 \mathrm{~mJ}$ or above. Figure 30 (b) confirms that the excitonic luminescence from $\mathrm{ZnO}$ nanowires improves from a course of surface etching and smoothing obtained during their exposure to $\mathrm{HNO}_{3}$ vapor.

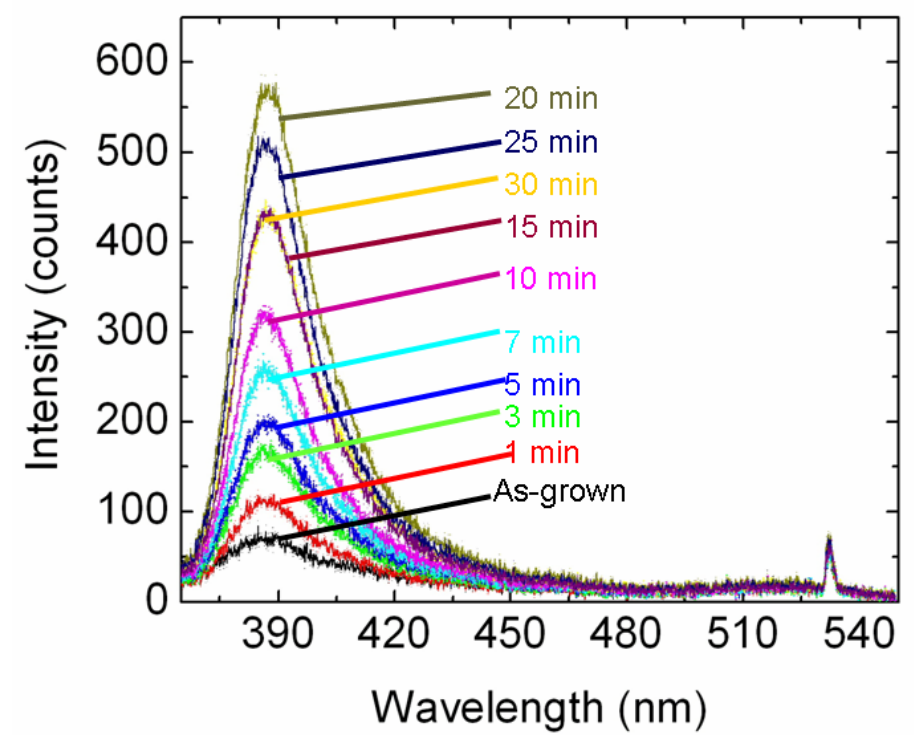

Fig. 28: Improvement of the excitonic luminescence in $\mathrm{ZnO}$ nanowires due to laser annealing with $10 \mathrm{~mJ}$ pulses incident directly onto the nanowires 


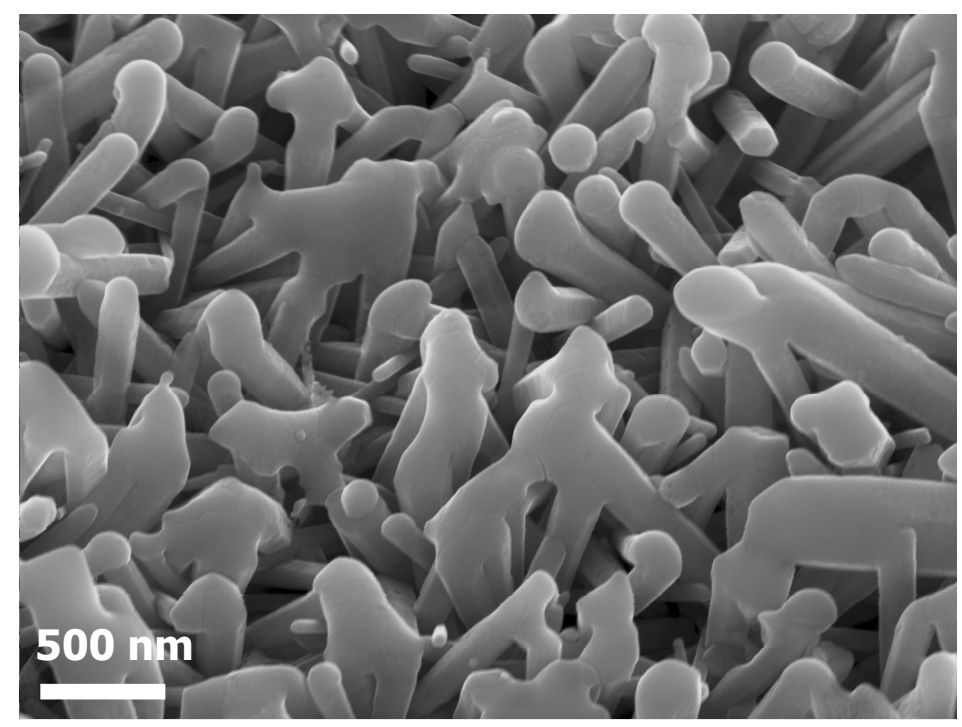

Fig. 29: Scanning electron micrograph showing the melted $\mathrm{ZnO}$ nanowires on $\mathrm{SnO}_{2}$ coated glass substrate after 30 min exposure to $10 \mathrm{~mJ}$ laser pulses
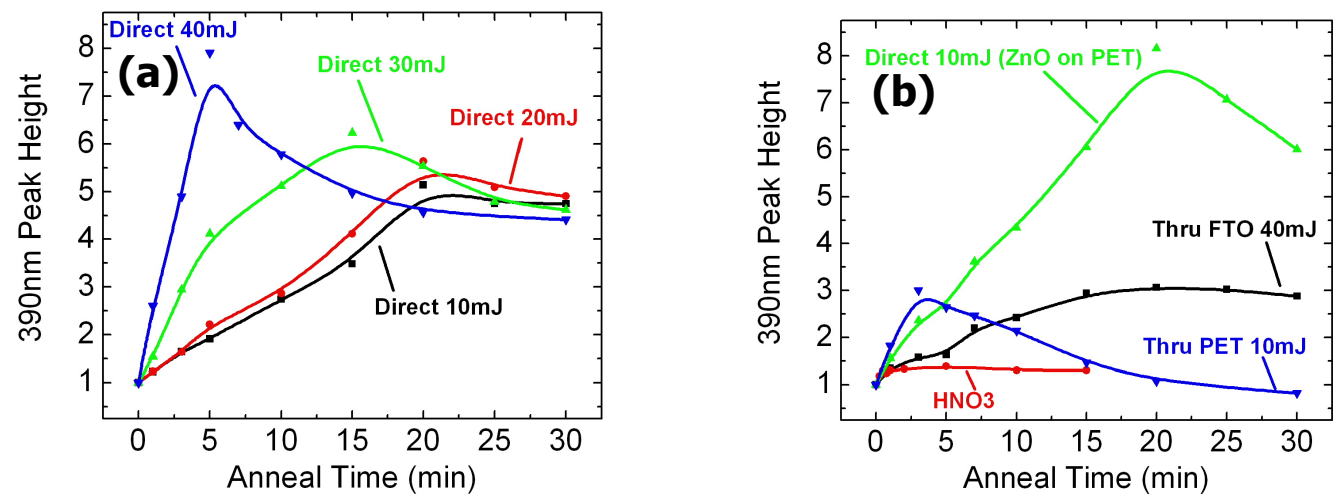

Fig. 30: Near band-edge photoluminescence at $390 \mathrm{~nm}$ as a function of laser anneal time at various laser pulse energies for (a) direct illumination on $\mathrm{ZnO} / \mathrm{FTO}$ and (b) illumination through various substrates 
We also measured the angular-dependent behavior of the PL spectra at roomtemperature. This measurement shows that the excitonic luminescence is guided preferentially, while the defect emission is emitted more isotropically, as shown in Figure 31. This finding is well agreed with by the results obtained by Johnson et al., 2003 [196]. They reported that the efficiency of coupling of the photoluminescence to waveguide modes varies roughly as an inverse of wavelength, resulting in shorter wavelengths are preferentially guided.

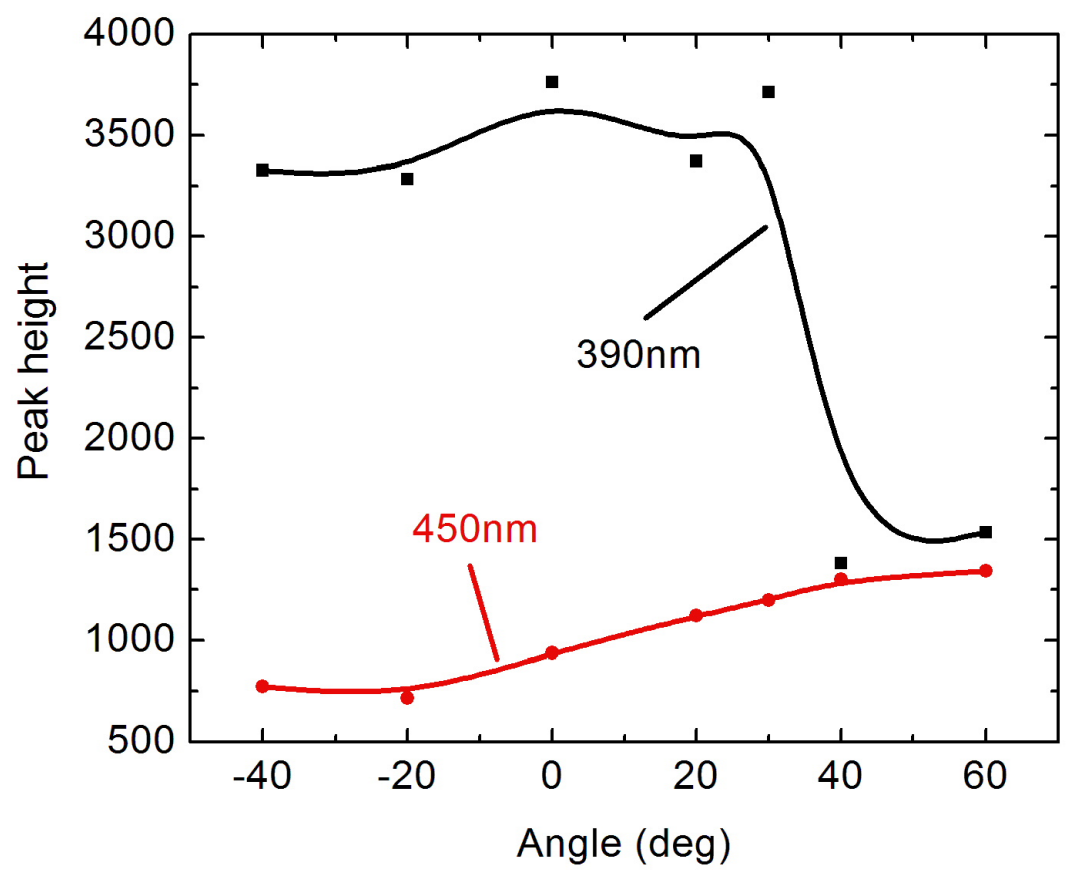

Fig. 31: Near-bandgap and sub-bandgap emission peaks as a function of detection angles 
4.2 Electroluminescence from $\mathrm{ZnO}$ nanowires/polymer light-emitting diodes

4.2.1 Light-emitting diodes on planar substrates

\subsubsection{Overview}

In order to obtain an electroluminescence from our $\mathrm{ZnO}$ nanowires, we fabricated hybrid p-n junction LED arrangements as described in detail in section 3.3. The electroluminescence of various LED structures made with as-grown and annealed nanowires, both undoped and doped, were studied. Ultraviolet electroluminescence was observed in annealed $\mathrm{Al}$-doped $\mathrm{ZnO}$ nanowires. Various luminescence bands covering the whole visible spectrum, and reaching into the near infrared region, were observed in $\mathrm{ZnO}$ nanowires doped with other impurities.

\subsubsection{Device structure}

The basic device configuration for these studies is illustrated in Figure 32. $\mathrm{ZnO}$ nanowires are grown on a FTO-covered glass substrate. The space between the nanowires is then filled with an insulating polystyrene layer. The back-contact is formed by a p-type polymer (PEDOT:PSS) and a gold back-contact. Several variations to the parameters of the basic components in this design were changed, as discussed in section 3.3.1, and their subsequent devices tested, over the course of this study. 


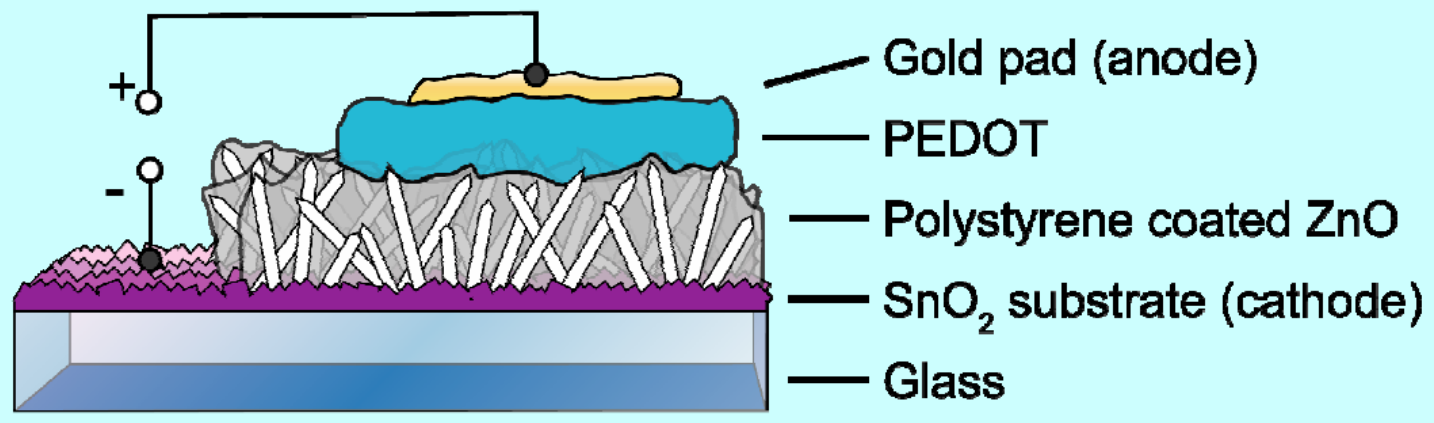

Fig. 32: Schematic diagram of a $\mathrm{ZnO}$ nanowire-based LED arrangement on a planar FTO/glass substrate

\subsubsection{Basic results of our nanowire LED}

Figure 33 shows the primary current-voltage and electroluminescence results of an LED made of undoped $\mathrm{ZnO}$ nanowires. As is evident in Figure 33 (a), the currentvoltage plot of this LED exhibits a reasonable PN junction characteristic with an excellent rectification behavior in reverse biased conditions. Electroluminescence was observed when the device was under forward biased conditions with a typical onset voltage of about 5-7 V. Figure 33 (b) shows the electroluminescence spectrum for this device at an applied voltage of $10 \mathrm{~V}$. This electroluminescence spectrum consists of a visible broad-band emission over the $450-900 \mathrm{~nm}$ region and a distinct shoulder extending into the UV region down to a wavelength of $360 \mathrm{~nm}$. This broad sub-bandgap emission in the electroluminescence spectrum, centered at $620 \mathrm{~nm}$, is due to 
recombinations via defects and surface states, whereas the UV emission, centered at 390 $\mathrm{nm}$, is attributed to an excitonic recombination $[12,13]$.
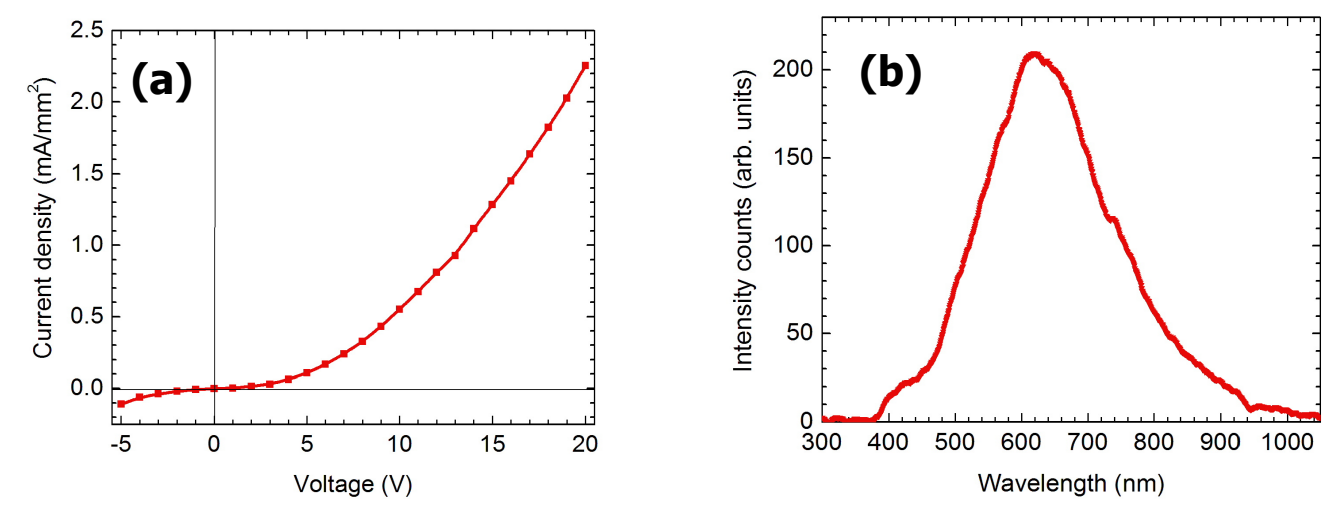

Fig. 33: (a) Current-voltage characteristics and (b) electroluminescence spectrum of undoped $\mathrm{ZnO}$ nanowire-based LED

\subsubsection{Operational principles of our nanowire LED}

Here, we use an energy band diagram in order to illustrate the basic operational principles of our LED. The schematic of this energy band diagram, with work function values for the nanowire's LED, is shown in Figure 34. Since our LED devices emitted light under forward bias, we can conclude that the electron can be injected from the $\mathrm{SnO}_{2} / \mathrm{ZnO}$ interface with the gold layer acting as a hole injection terminal. Those two injected carriers then are recombined in the $\mathrm{ZnO}$ to produce the electroluminescence. There is a $2.4 \mathrm{eV}$ barrier for hole injection from the HOMO level of PEDOT:PSS to the $\mathrm{ZnO}$ valence band edge and a further $0.7 \mathrm{eV}$ barrier between the $\mathrm{SnO}_{2}$ Fermi energy level and the $\mathrm{ZnO}$ conduction band edge. Therefore, the carrier injection requires a 
potential of at least $3.1 \mathrm{~V}$ in the case of forward bias conditions. Choosing the proper contact materials for the design is crucial to obtaining devices with a substantial reduction of these barriers. They can also be reduced by utilizing a sequence of small energy barrier multilayer contacts. These refinements could potentially lead to enhancement of the luminescence's onset voltage and current density in this type of device.

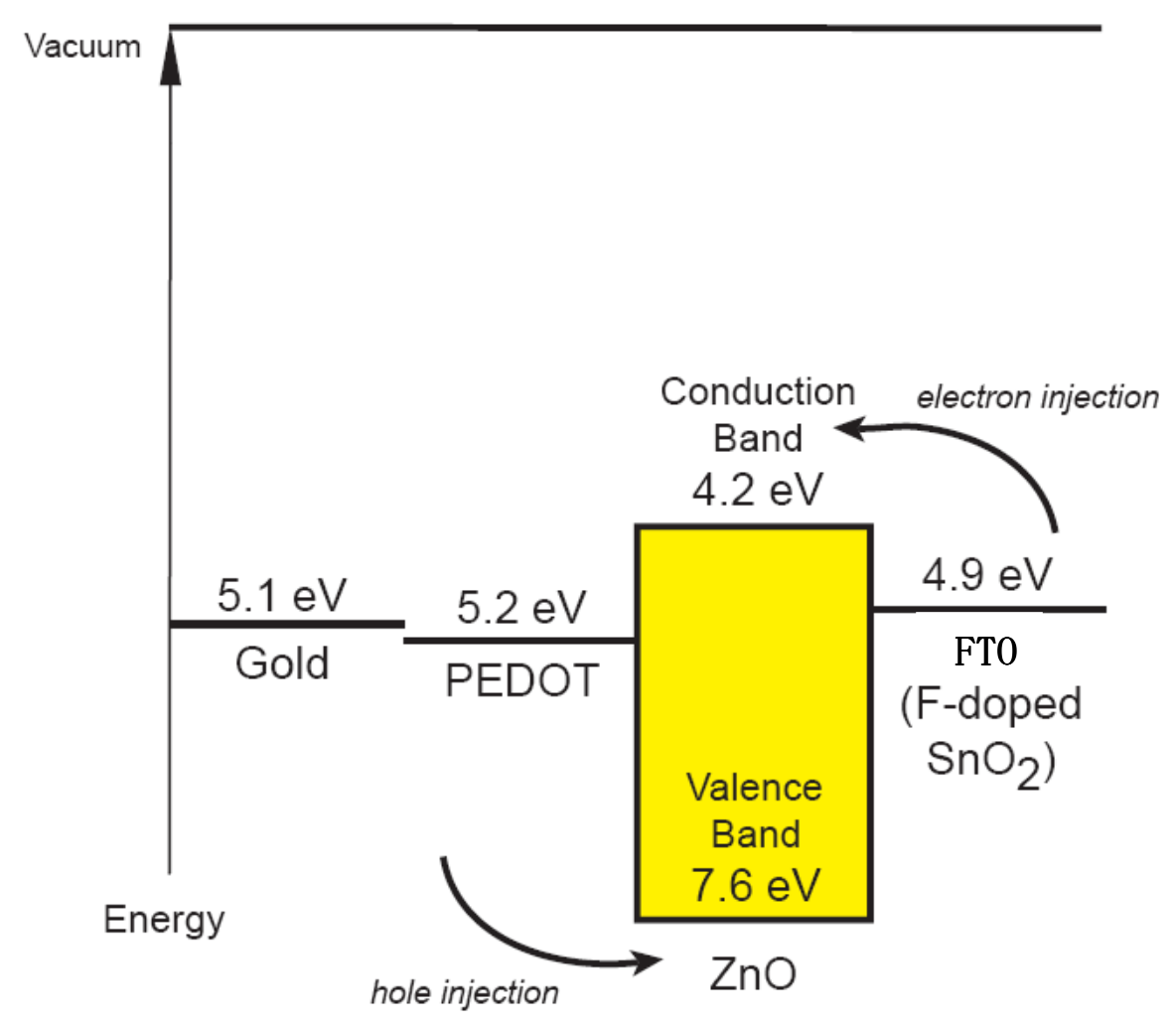

Fig. 34: Band energy levels of the nanowire/polymer LED device arrangement 
4.2.1.5 Electroluminescence of as-grown and annealed $\mathrm{ZnO}$ nanowires

\subsection{Electroluminescence of as-grown nanowires}

The obtained electroluminescence spectra from as-grown nanowires doped with various metal ions showed a weak excitonic emission peak, as well as a dominant broad emission band in the visible range (See Figure 35). This visible broad emission band is due to either surface defects or the recombination via distributions of different defect states, or a combination of their contributions. We can see that in un-annealed nanowires the peak of visible emission, centered at $620 \mathrm{~nm}$, shows no shifts in wavelength regardless of doping. Thus, it is clear that in the as-grown state the doping has only limited effects on the optical properties, since intrinsic defects dominate the structure and determine the properties of the nanowires. From our luminescence studies,

we can conclude that the strain induced defects present in an un-annealed state are strongly dominant over any dopant-related effects. 


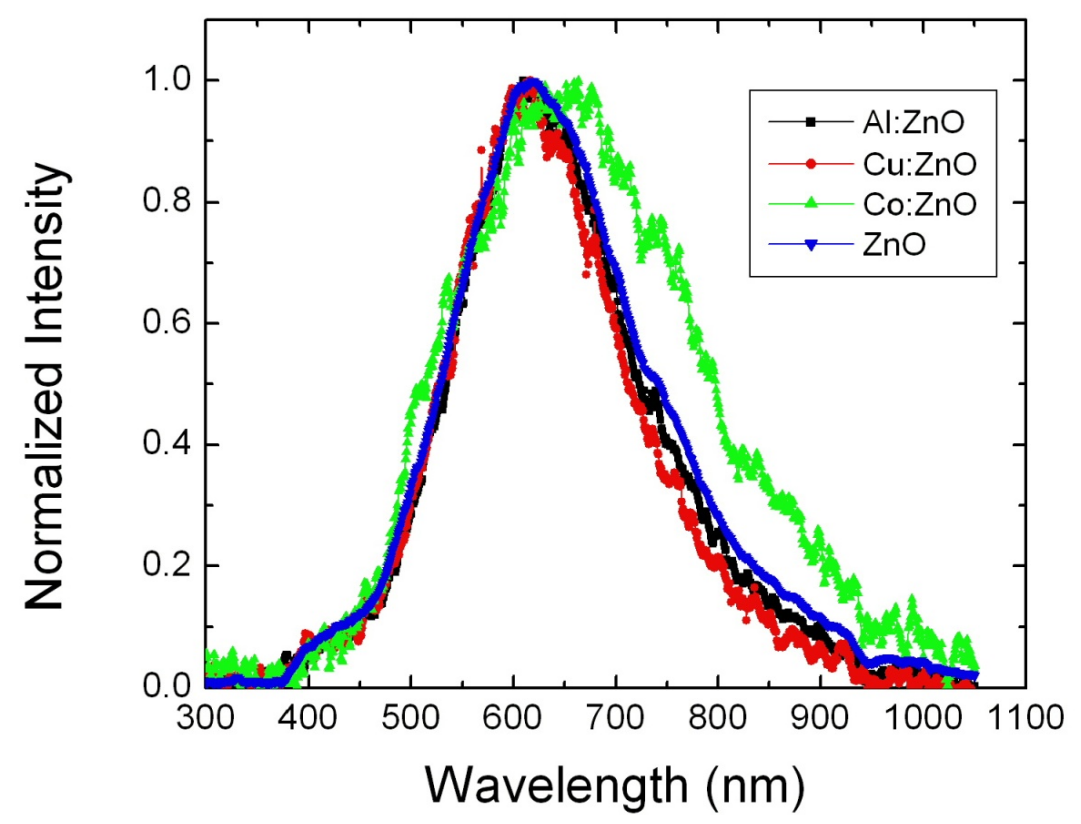

Fig. 35: Electroluminescence spectra of various transition-metal-ion doped as-grown $\mathrm{ZnO}$ nanowire LED devices

We observed a slight reduction of blue and ultra-violet contributions in the electroluminescence as compared to the photoluminescence for our as-grown $\mathrm{ZnO}$ sample, as shown in Figure 36. The differences between electroluminescence and photoluminescence are due to the reduction of the initial energies of the injection carriers. In electroluminescence, these initial energies are reduced by trapping processes, whereas in photoluminescence the initial excitation energy is much higher than the bandgap of $\mathrm{ZnO}$. Thus, even from the beginning the carriers have enough spatial overlap to have an effective high energy related recombination, like excitonic, band-toband, or donor-acceptor transitions. 


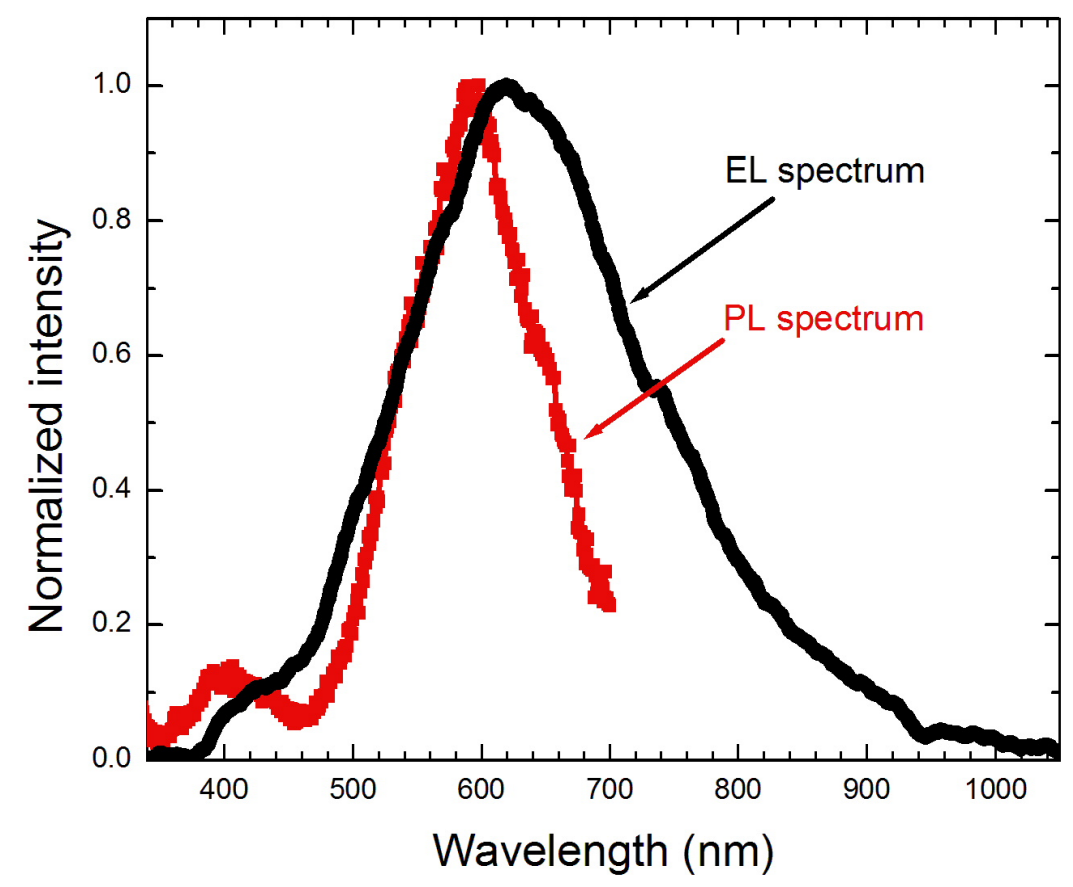

Fig. 36: PL and EL spectra obtained from as-grown $\mathrm{ZnO}$ nanowires

\subsection{Electroluminescence of annealed nanowires}

The electroluminescence spectra of the annealed $\mathrm{ZnO}$ nanowires clearly reveals that the defect-related peak shifts from $620 \mathrm{~nm}$ to a higher wavelength value depending on the type of dopant, as shown in Figure 37. We can also see that the additional narrow UV emission line, with an improved intensity, is observed in LEDs made of Al doped $\mathrm{ZnO}$ nanowires annealed at $380^{\circ} \mathrm{C}$. When annealed at $380^{\circ} \mathrm{C}$, intrinsic defects in the nanowires are reduced significantly in their density; hence the luminescence efficiency increases and the impurity-related transitions become more dominant. From our photo- 
and electroluminescence studies, we can conclude that moderate temperature thermal annealing, as well as laser annealing, induces strain relaxation and the associated dopant activation, such that the optical and electrical properties of the nanowires become suitable for the fabrication of devices such as solar cells and LEDs.

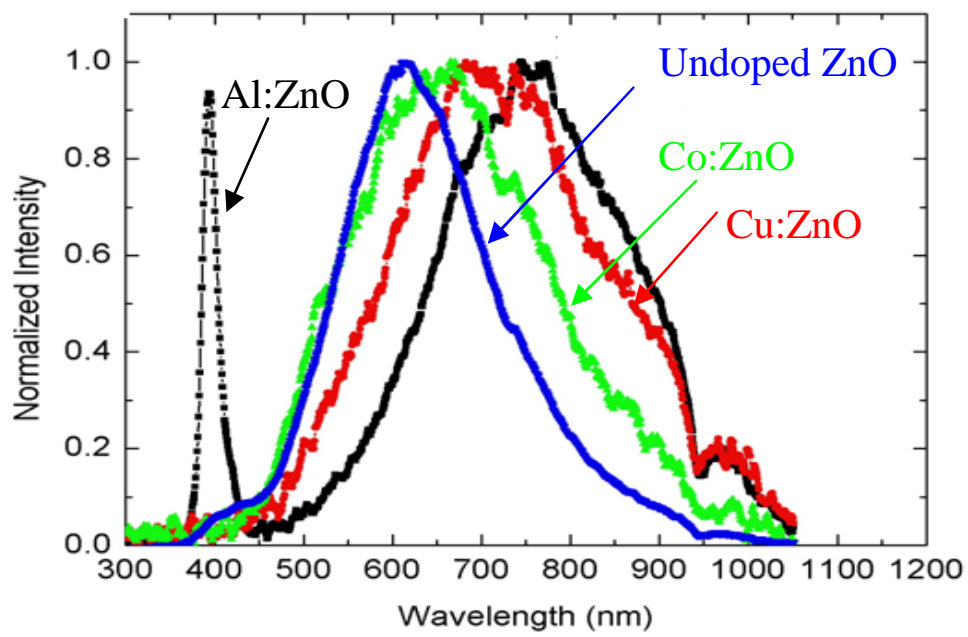

Fig. 37: Electroluminescence spectrum of various transition-metal-ion doped $380^{\circ} \mathrm{C}$ annealed $\mathrm{ZnO}$ nanowire LED devices, which demonstrate that the visible luminescence is dominated by metal impurities

Figure 38 shows the comparison of the electroluminescence and the photoluminescence spectrum of $\mathrm{Al}$ doped $\mathrm{ZnO}$ nanowire film, annealed at $380^{\circ} \mathrm{C}$, revealing that the blue and ultra-violet contributions are rather reduced in the electroluminescence spectrum. The electroluminescence and photoluminescence are different mainly due to the following characteristics: In electroluminescence, the initial energies of the injected carriers are near or even below the $\mathrm{ZnO}$ band edge energies. 
These initial energies are then further reduced during the drift of carriers into the sample bulk by trapping processes produced by defects present in the $\mathrm{ZnO}$ nanowire. This causes only a very small portion of the carriers to have sufficient energy for excitonic transitions to occur. It is only when the injected electron distribution and hole distribution have gained sufficient spatial overlap that they are capable of producing effective recombination. In the case of photoluminescence, the laser excitation energy is $3.8 \mathrm{eV}$, which is much larger than the bandgap energy of $\mathrm{ZnO}$, and therefore the electrons and holes have enough initial spatial overlap for recombination to be immediately effective. There is then a much larger probability of the occurrence of higher energy transitions. Thus, excitonic transitions, band-to-band transitions, and donor-acceptor pair transition rates are more prominent in the photoluminescence process. This strong difference between electro- and photoluminescence spectra indicates that carrier transport remains a serious challenge to create efficient LEDs for practical lighting applications. However, it appears likely that shorter transport path length and the utilization of tunneling barriers at the injecting contacts will improve the efficiencies of LEDs. 


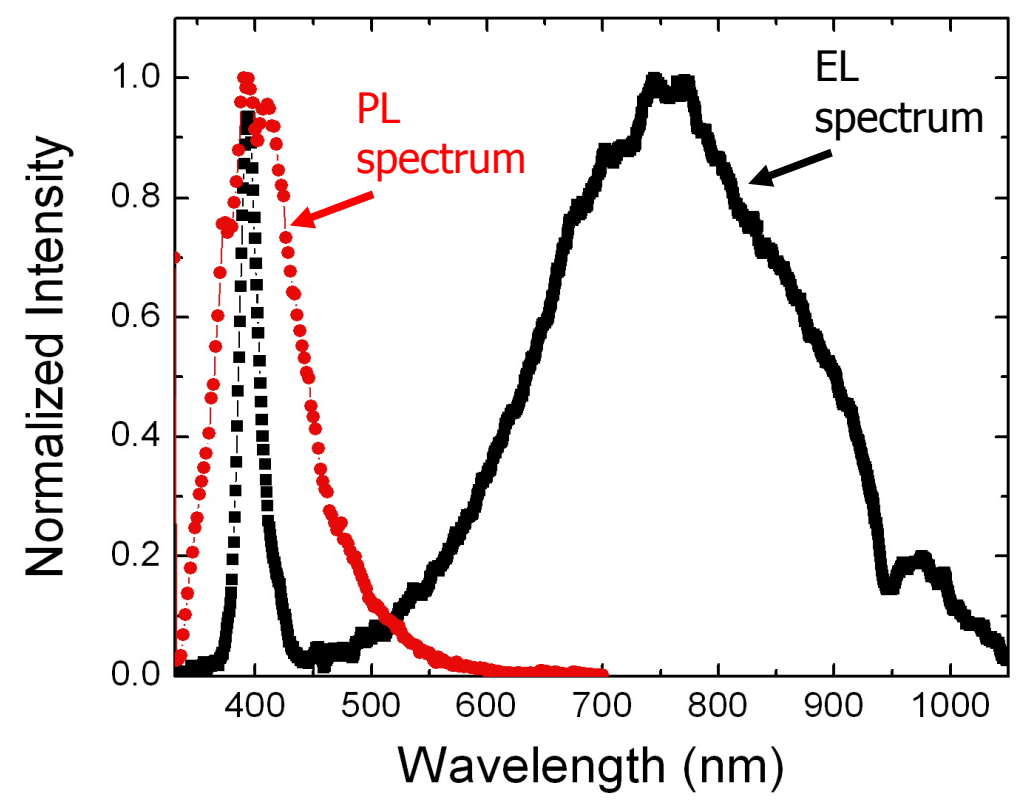

Fig. 38: PL and EL spectra obtained from $\mathrm{Al}$ doped $\mathrm{ZnO}$ nanowires annealed at $380^{\circ} \mathrm{C}$. The PL is obtained with excitation of a He-Cd laser at $325 \mathrm{~nm}$, and the EL is obtained with excitation voltage of $10 \mathrm{~V}$, at room temperature in air

\subsubsection{Light-emitting diodes on flexible substrates}

\subsubsection{Overview}

In this section, we describe a novel light-emitting diode structure grown on a flexible, PET foil, and embedded in a polymeric matrix [15]. The PET foil is transparent, and covered with a conductive ITO layer, such that the same design layout on glass, as described in section 4.1.1, can be used. The flexibility makes it an interesting alternative to all-organic electronic and photonic devices. A broad emission 
spectrum, covering most of the visible range and reaching to the near-ultraviolet, was obtained from this flexible hybrid light emitting diode.

\subsubsection{Device structure}

Figure 39 shows the schematic representation of a $\mathrm{ZnO}$ nanowire LED on a flexible, transparent ITO/PET substrate. The optically active component in this device is an inorganic single-crystalline $\mathrm{ZnO}$ nanowire film, while the polystyrene film provides a robust yet flexible support matrix.

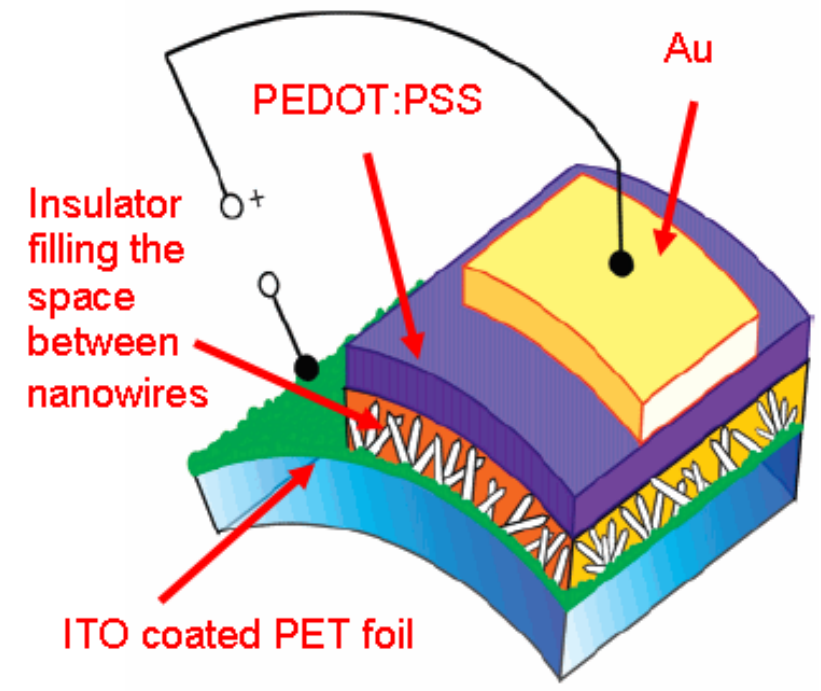

Fig. 39: Schematic diagram of a $\mathrm{ZnO}$ nanowire-based LED arrangement on a flexible substrate 


\subsubsection{Morphology of $\mathrm{ZnO}$ nanowires on flexible substrates}

Figure 40 shows the SEM micrograph of a typical $\mathrm{ZnO}$ nanowire film grown on a transparent, flexible PET foil. These nanowires were grown at a slightly higher negative potential of $-1.05 \mathrm{~V}$, as compared to those grown on FTO/glass substrates. Therefore, the nanowires grown on ITO/PET substrates are around $1 \mu \mathrm{m}$ in length and 70-120 nm in diameter, making them relatively shorter and thinner than those obtained from planar FTO/glass substrates. A polystyrene solution with slightly less concentration $(20 \mathrm{~g} / \mathrm{L})$ was then spin-coated in order to completely fill the space between the nanowires and to produce a very thin coverage on the nanowire tops, as shown in Figure 41.

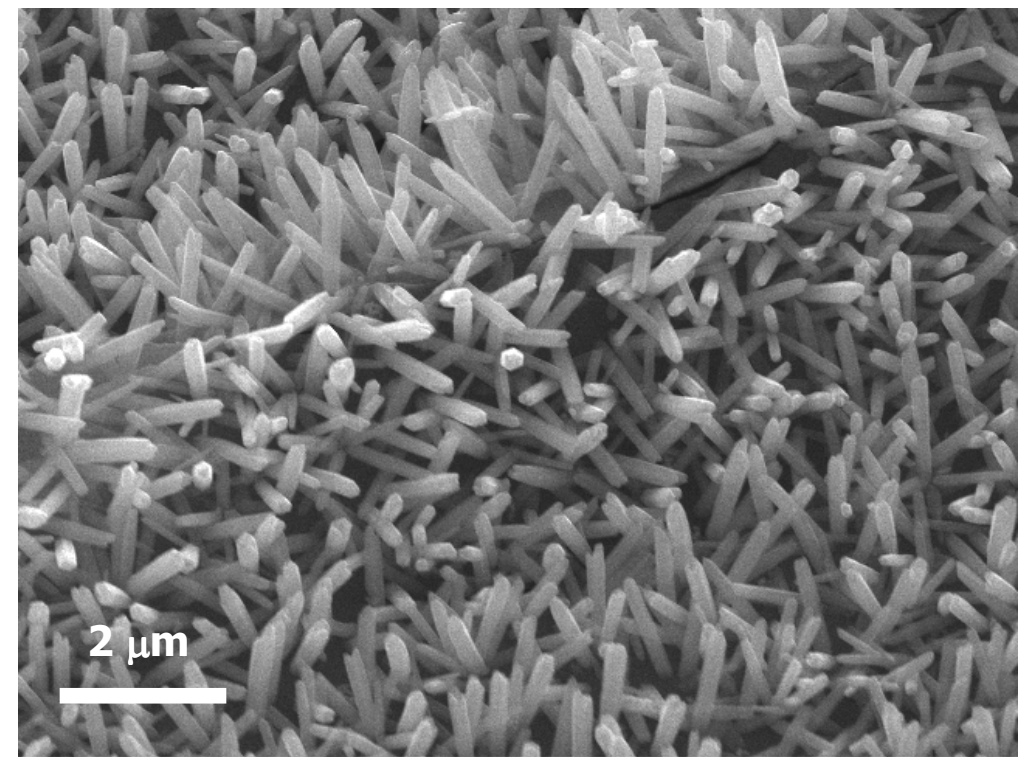

Fig. 40: The SEM image of $\mathrm{ZnO}$ nanowires grown in electrodeposition on a flexible transparent ITO/PET substrate 


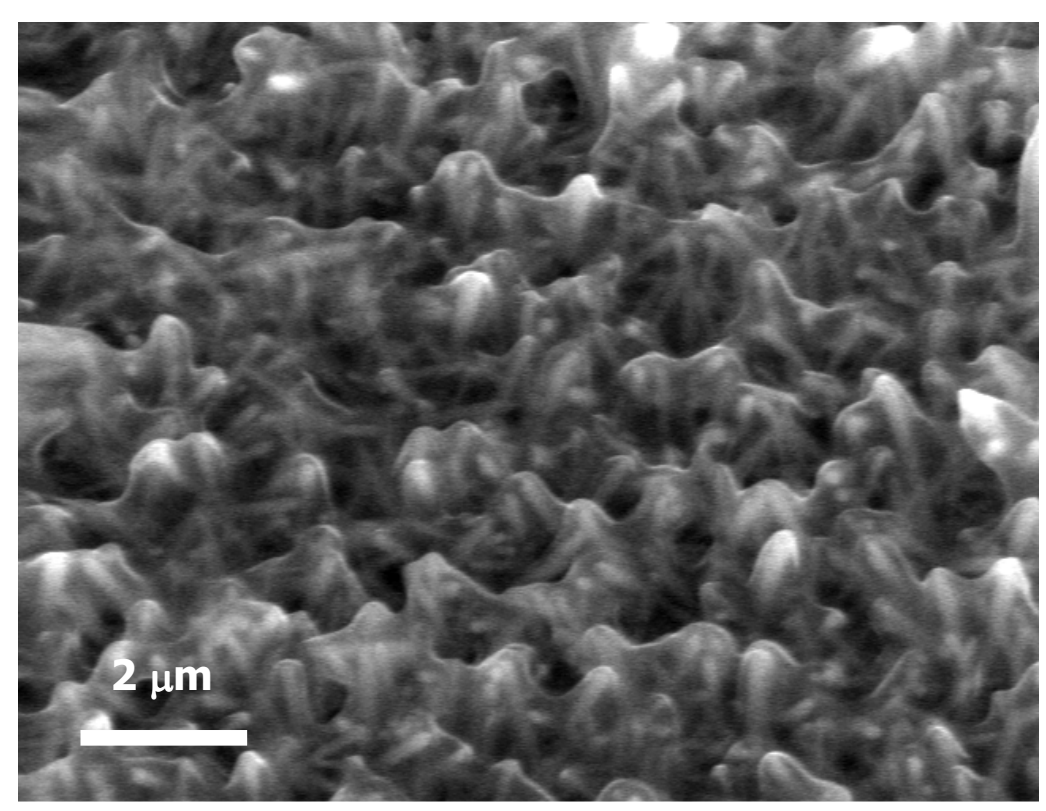

Fig. 41: $\mathrm{ZnO}$ nanowires spin-coated with insulating layer from a polystyrene/toluene solution

4.2.2.4 Current-voltage characteristics of as-grown and annealed $\mathrm{ZnO}$ nanowires

Figure 42 illustrates the current-voltage characteristics of the flexible and the planar LED devices. As can be seen in Figure 42, the rectification was mainly produced at the back contact of the $\mathrm{n}^{-}-\mathrm{i}-\mathrm{p}^{+}$heterojunction, which has a layer sequence of $\mathrm{ZnO} / \mathrm{PS} / \mathrm{PEDOT}: \mathrm{PSS} / \mathrm{Au}$. This back contact is identical for flexible and planar LED devices. However, the forward bias current in the flexible LED device is higher than that obtained from planar FTO/glass LED device. We can see that the $\mathrm{n}^{-}-\mathrm{i}-\mathrm{p}^{+}$ heterojunction limits the forward currents only for voltages below approximately $3 \mathrm{~V}$. 


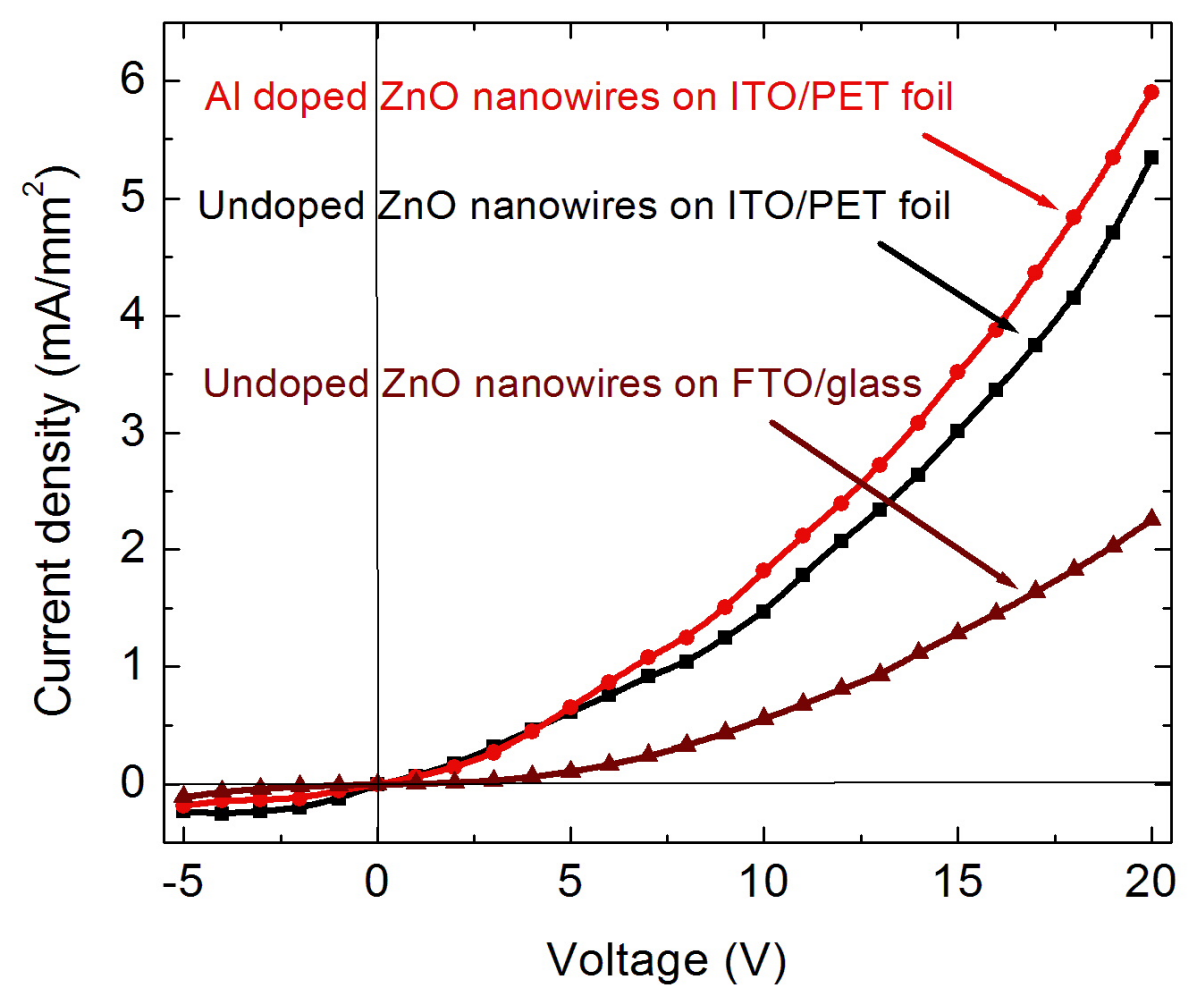

Fig. 42: The electrical characteristics of various LED structures

\subsubsection{Electroluminescence of as-grown and annealed $\mathrm{ZnO}$ nanowires}

Figure 43 shows the electroluminescence spectra for undoped and Al-doped nanowires on a flexible ITO-coated PET substrate in comparison to spectra obtained on a planar $\mathrm{SnO}_{2}$-coated glass substrate. The spectra from the flexible devices showed slightly less intensity as compared to planar LED devices. However, the emission spectrum of these flexible LEDs is qualitatively similar to those on planar substrates. We can also see that the defect-related peak was slightly red-shifted from the spectra 
obtained on the glass substrates. Defect transitions in the $\mathrm{ZnO}$ are the likely origin of this sub-bandgap emission. This transition can be tuned in a controlled manner by adding impurities to the electrolyte solutions. We found that Al-doping in the range of $5 \mu \mathrm{M}$ further increases the red-shift as compared to the undoped $\mathrm{ZnO}$ nanowire defectrelated emission spectra. Previous studies on planar LEDs show that the annealing of Al-doped nanowires at $380^{\circ} \mathrm{C}$ increases the emission intensity and produces an ultraviolet emission peak around $390 \mathrm{~nm}$ [209]. The PET foils limit the thermal annealing to temperatures up to $150^{\circ} \mathrm{C}$, and the ultraviolet emission was therefore not observed. An ultraviolet near band-gap emission from these low-temperature growth nanowires can be obtained after annealing at $\sim 300^{\circ} \mathrm{C}$. This temperature is still in the stability range of some polymer-based substrates, such as Kepton [211]. Overall, this device presents an interesting alternative to organic LEDs because the optically active material in our device is inorganic, leading to less critical stability issues than those found in devices constructed using all-organic materials. 


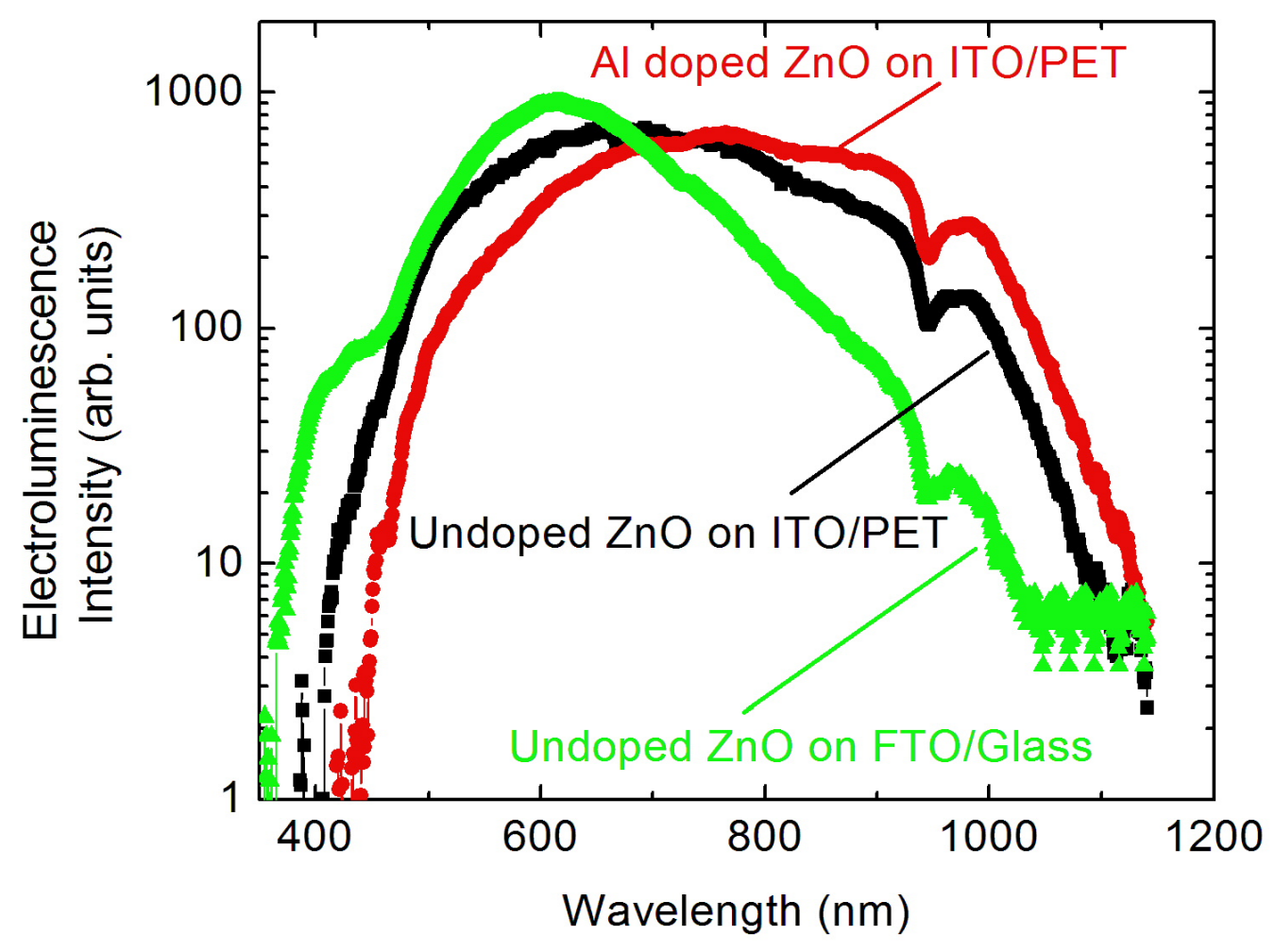

Fig. 43: Electroluminescence spectra of flexible LEDs as compared to a planar LED 
4.3 Nanowire-quantum-dot solar cells and their performances

\subsubsection{Overview}

In this section, we describe an innovative type of nanowire-quantum-dot solar cell which utilizes $\mathrm{CdSe}$ quantum dots deposited on $\mathrm{ZnO}$ nanowires to convert light into electrical current flow [86]. We also describe how $\mathrm{CdCl}_{2}$ thermal annealing plays an important role in improving device performance. In order to understand the structural changes that occur during the annealing process, we studied the crystal properties of the $\mathrm{ZnO} / \mathrm{CdSe}$ interface, using electron microscopes, before and after the $\mathrm{CdCl}_{2}$-aided thermal treatment. We observed a structural conversion of CdSe quantum dots from their initial loosely-connected particle arrangement into a poly-crystalline thin film with high surface conformality. The enhanced photovoltaic performance of annealed devices can be attributed to this structural conversion. We also explored how the use of different polymer back contacts affects the performance of nanowire-quantum-dot solar cells: P3HT was found to be the best back contact layer for our purposes. Furthermore, we studied how the formation of compact $\mathrm{ZnO}$ thin film on the FTO/glass substrate prior to the electrochemical growth of $\mathrm{ZnO}$ nanowires improves the performance of the solar cell. Most recently, we constructed solar cells using CdSe quantum dots surfacetreated with pyridine solution and EDT ligand exchange instead of as-synthesized quantum dots. This chemical treatment resulted in greatly improved photovoltaic performances in our devices, and gave a best energy conversion efficiency of $3.4 \%$, 
which is comparable to the best reported efficiencies for this type of solar cell to date [197].

\subsubsection{Why nanowires?}

The $\mathrm{ZnO}$ nanowires provide for an enlargement of the surface area, allowing for a reduction of the local thickness of the absorber layer in our solar cells. This surface enlargement also presents significant advantages for light absorption and charge separation, which are two critical steps in solar-to-electric energy conversion. The morphology and single-crystalline nature of $\mathrm{ZnO}$ nanowires provide for a direct pathway for photo-generated electrons to transfer from the active absorber layer to the FTO contact, thereby reducing the length and time needed for these photo-generated carriers to diffuse. The geometry of $\mathrm{ZnO}$ nanowires provide nanowire based solar cells with a potential advantage over planar or thin film based solar cells due to the multiple reflections of light that occur inside the cell, which allows the photons to travel further in the material, thereby improving light trapping and increasing light absorption.

\subsubsection{Why quantum dots?}

Quantum dots synthesized by colloidal chemistry and suspended in solution offer several advantages: First, their bandgap can be easily adjusted by changing the size of the particle to match the solar spectrum. Second, they can be simply prepared as an ink and deposited as a very thin absorber on a deeply nanostructured sample by 
simple process, like printing, in order to reduce the amount of material needed for absorption. Additionally, there is a possibility that multiple electron-hole pairs generated by multiple-exciton generation [198], hot carrier collection [199], and singleexciton fission [200] processes, contribute to further improvements of the solar cell, moving the energy conversion efficiency beyond the Shockley-Queisser theoretical limit of $31 \%$.

\subsubsection{Why a surface treatment for the quantum dot material?}

The selection of the proper type of ligand molecules for use in the colloidal synthesis of quantum dots is the key parameter for achieving samples with a narrow size distribution and in controlling their shape. Fatty acids, long chain amines, phosphines, and phosphonic acids have been found to be suitable ligand molecules for controlling the structural properties of quantum dots. In our case, as-synthesized CdSe quantum dots are capped with long chain ligand molecules of octadecylamine in order to prevent their agglomeration. However, this native ligand molecule obstructs charge transport, producing a thin insulating film. When creating conductive quantum-dot films, it is necessary to replace the CdSe quantum dots' initial long chain ligand molecules with short chain ligand molecules in order to provide a better pathway for transferring the photogenerated charge carriers within the CdSe quantum dot layers and to the contacting material. 


\subsubsection{Device structure}

The basic schematic representation of our nanowire-quantum-dot solar cell is illustrated in Figure 44. An array of $\mathrm{ZnO}$ nanowires were grown vertically on a transparent conducting glass substrate, as detailed in section 3.1.1. Nanometer-size colloidal CdSe quantum dots were attached to the surface of the nanowires, as described in section 3.4.3.2. These deposited quantum dots were converted from their initial loosely connected particle arrangements into thin continuous poly-crystalline films with typical grain diameters of $30-50 \mathrm{~nm}$ by a $\mathrm{CdCl}_{2}$-aided thermal anneal process. The back contacts were made of a spin/drop-coated hole-conducting material and a thermally evaporated Au layer. Various modifications of the cell components were carried out over the course of this study in order to optimize the energy conversion efficiency.

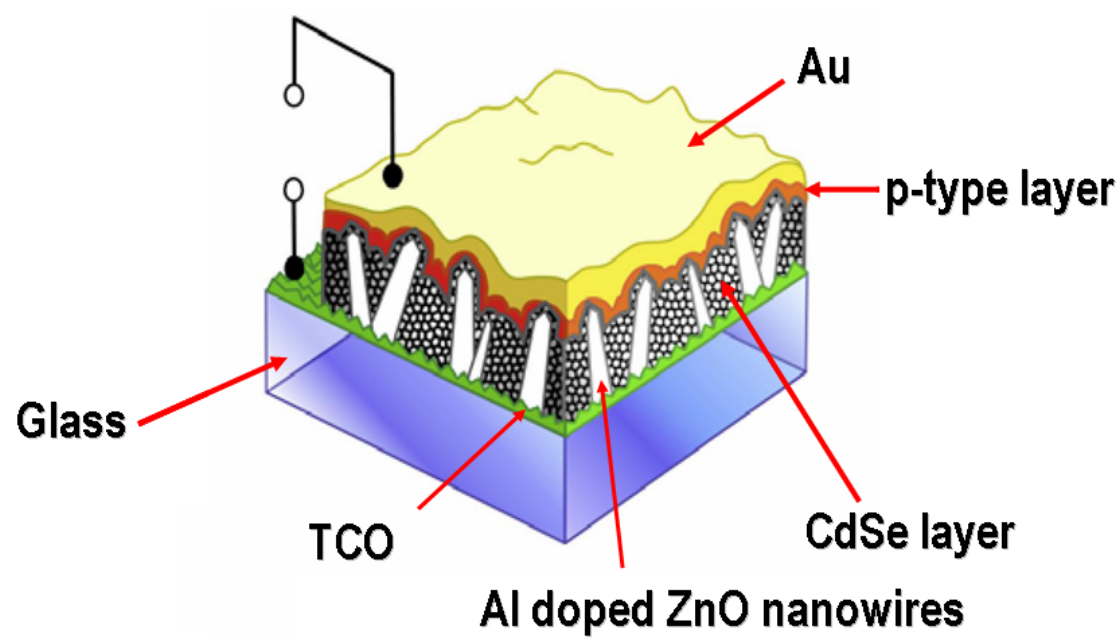

Figure 44: Schematic layout of our nanowire-quantum-dot solar cell 


\subsubsection{Concepts of extremely thin absorber solar cells}

Our devices were designed by following the concepts behind the architecture of the extremely thin absorber solar cell $[119,201]$. In this design, very thin CdSe quantum-dot absorber material is placed between the n-type transparent $\mathrm{ZnO}$ nanowires and a p-type polymer layer. The enlarged surface area provided by the nanowires increases the effective absorber layer thickness, as well as offering excellent light trapping. The local thickness of the absorber material has to be thicker than the tunneling length in order to prevent shunting between the electron and hole conductors, but must remain thinner than the diffusion length of photo-generated charge carriers.

\subsubsection{Primary results of our solar cells}

\subsubsection{The un-annealed solar cell}

Figure 45 (a) represents the external quantum efficiency plot as a function of wavelength for the un-annealed nanowire-quantum-dot solar cell. This plot indicates that the response comes only from the polymer back contact, showing an absorption edge around $550 \mathrm{~nm}$, which is consistent with polymer absorption. A photoresponse from the $7 \mathrm{~nm}$ CdSe quantum dots could not be detected. It appears that the long chain ligand molecules that cap the CdSe quantum dots prevent the agglomeration and block the transport of photogenerated carriers within the quantum dot layers, as well as their transport from quantum dot layer to neighboring layers. However, later in section 4.3.9, 
we used chemically surface cleaned CdSe quantum dots treated by EDT ligand exchange in the preparation of nanowire-quantum-dot solar cells. These solar cells exhibited an improved photo-response with the onset position of the photo-response spectrum shifting towards the $7 \mathrm{~nm}$ CdSe quantum dot bandgap energy value of $1.9 \mathrm{eV}$, corresponding to a wavelength value of $650 \mathrm{~nm}$, as shown later in Figure 55. The light and dark current-voltage characteristics of the un-annealed nanowires-quantum-dot solar cell made with initial ligand molecules capped CdSe quantum dots are shown in Figure 45 (b). This device exhibited a short-circuit current density $\left(J_{\mathrm{SC}}\right)$ of $0.8 \mu \mathrm{A} / \mathrm{cm}^{2}$ and an open-circuit voltage $\left(V_{\mathrm{OC}}\right)$ of $0.15 \mathrm{~V}$ with a very low energy conversion efficiency of $0.0043 \%$.
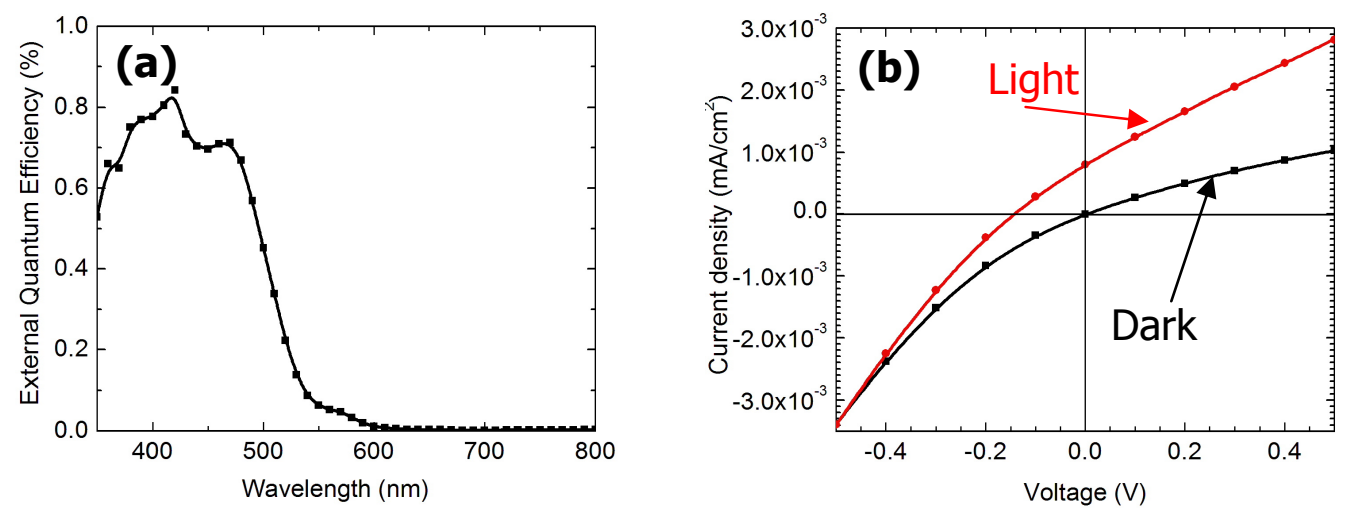

Fig. 45: (a) spectral response and (b) light and dark current-voltage characteristic plots of our un-annealed nanowire-quantum-dot solar cell 
4.3.4.2 Solar cell performances after two hour $\mathrm{CdCl}_{2}$-aided thermal annealing process

In order to remove the ligand molecules, we annealed the solar cell at $380^{\circ} \mathrm{C}$ in $\mathrm{CdCl}_{2}$ /air, as described in section 3.4.3.3. As can be seen in Figure 46 (a), the photoresponse of the annealed solar cell increases drastically as compared to that of an un-annealed solar cell. The external quantum efficiency plot of the annealed sample also indicates that the photo-response spectrum onset position moves down in energy towards the CdSe bulk bandgap value of $1.74 \mathrm{eV}$, corresponding to a wavelength of 712 nm. This change in the onset position of the absorption spectrum is due to the structural conversion of quantum dots into a polycrystalline film, as detailed in section 4.3.7.

The current-voltage characteristics (see Figure 46 (b)) also show much improved photovoltaic properties, resulting in an excellent rectification behavior as compared to that of un-annealed solar cells in reverse-biased and dark conditions. Enhanced photovoltaic performance was also achieved from the annealed solar cell under illumination with an open circuit voltage of $0.49 \mathrm{~V}$, a short circuit current of 1.9 $\mathrm{mA} / \mathrm{cm}^{2}$, and an energy conversion efficiency of $0.35 \%$. Annealing of CdSe quantum dots in $\mathrm{CdCl}_{2}$ /air clearly reduces the series resistance of the solar device, producing a 2.5-times greater open-circuit voltage $\left(V_{\text {oc }}\right)$, 2000-times greater short-circuit photocurrent density $\left(J_{\mathrm{sc}}\right)$, and 80-times greater energy conversion efficiency in the cell. In the un-annealed device, the octadecylamine molecules that cap the CdSe quantum dots resulted in a high series resistance (See Figure 45 (b)). This ligand molecule is a non-conductive material, and so acts as an insulating barrier effectively preventing 
photogenerated electrons and holes from reaching the $\mathrm{ZnO}$ layer and the p-type back contact, respectively. The improvement in performance of the annealed cell results from the removal of the ligand component of the CdSe quantum dot, and the conversion of the isolated quantum dots into a thin polycrystalline film. The annealing produces a CdSe film, in which the quantum dots are still visible, and that is thin enough to reduce the recombination process.
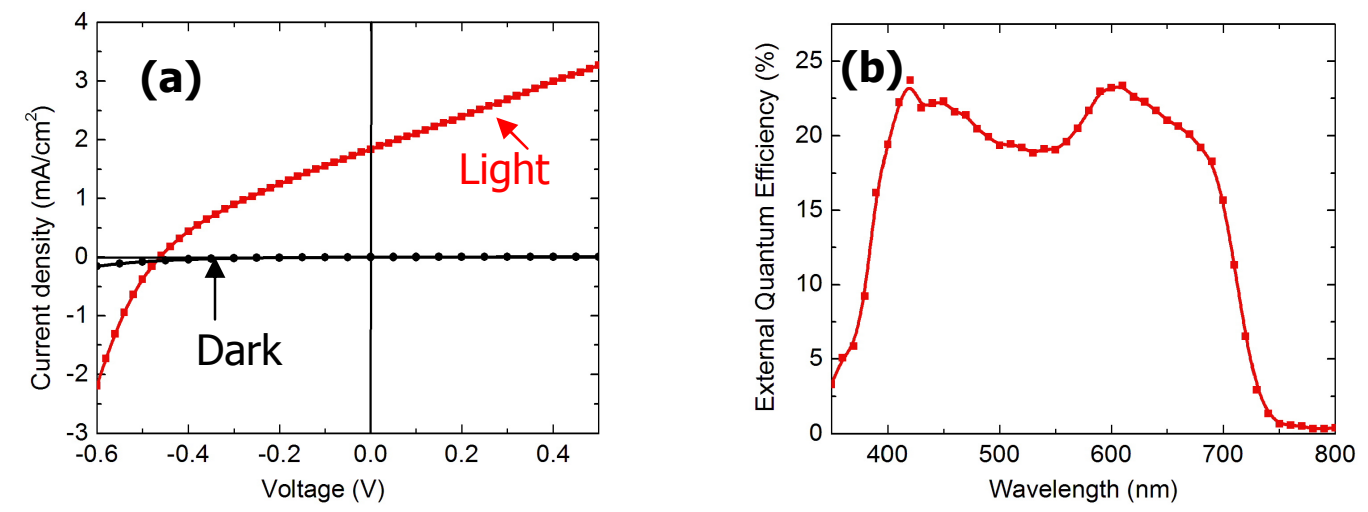

Fig. 46: (a) Current-voltage plot and (b) photoresponse behavior as a function of wavelength for a nanowire-quantum-dot solar cell annealed at $380^{\circ} \mathrm{C}$ in the presence of $\mathrm{CdCl}_{2}$ /air for two hours

\subsubsection{Effect of extended $\mathrm{CdCl}_{2}$-aided thermal annealed solar cells}

Furthermore, we found that the photo-response increases noticeably with extended anneal times, and that an anneal time of 24 hours leads to maximum quantum efficiency, as shown in Figure 47. However, annealing beyond this 24 hour period results in a decrease of the quantum efficiency [212]. 


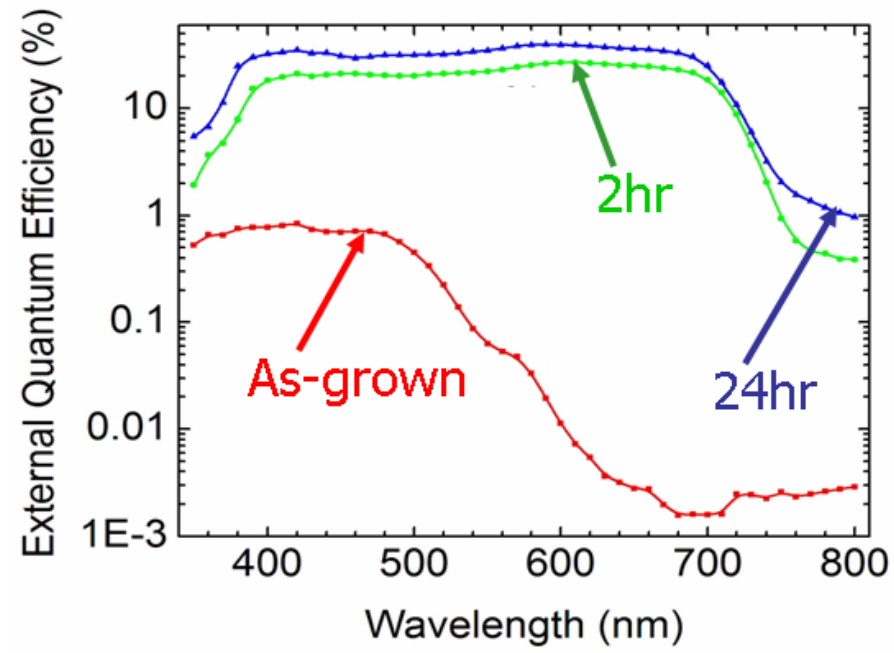

Fig. 47: Improvement of solar cell performance by increasing anneal times for annealing at $380^{\circ} \mathrm{C}$ in $\mathrm{CdCl}_{2}$ /air

\subsubsection{Operational principles of our solar cell}

Figure 48 shows the energy band diagram of the nanowire-quantum-dot solar cell with the corresponding valance and conduction energy values of each material component. In our solar cell design, we chose $\mathrm{ZnO}$ nanowires as an n-type electroncollecting window material, CdSe quantum dots as an absorber material, and P3HT as a p-type polymer for hole collection. CdSe has a bandgap of $1.7 \mathrm{eV}$ with a suitable conduction band energy level of $3.8 \mathrm{eV}$, allowing for the transfer of photo-generated electrons to the $\mathrm{ZnO}$ conduction band, and valence band energy level of $5.5 \mathrm{eV}$, which enables the transfer of photo-generated holes to the P3HT HOMO level. The $0.4 \mathrm{~V}$ value of the $\mathrm{ZnO}$ and $\mathrm{CdSe}$ conduction-band offset is considered to be an efficient 
barrier to electron injection from the $\mathrm{CdSe}$ to the $\mathrm{ZnO}$, and the offset value of $0.5 \mathrm{eV}$ for the CdSe valance band energy and P3HT HOMO level promotes hole transport from the CdSe to the P3HT. Therefore, the $\mathrm{ZnO} / \mathrm{CdSe} / \mathrm{P} 3 \mathrm{HT}$ heterostructure enforces the separation of charge carriers.

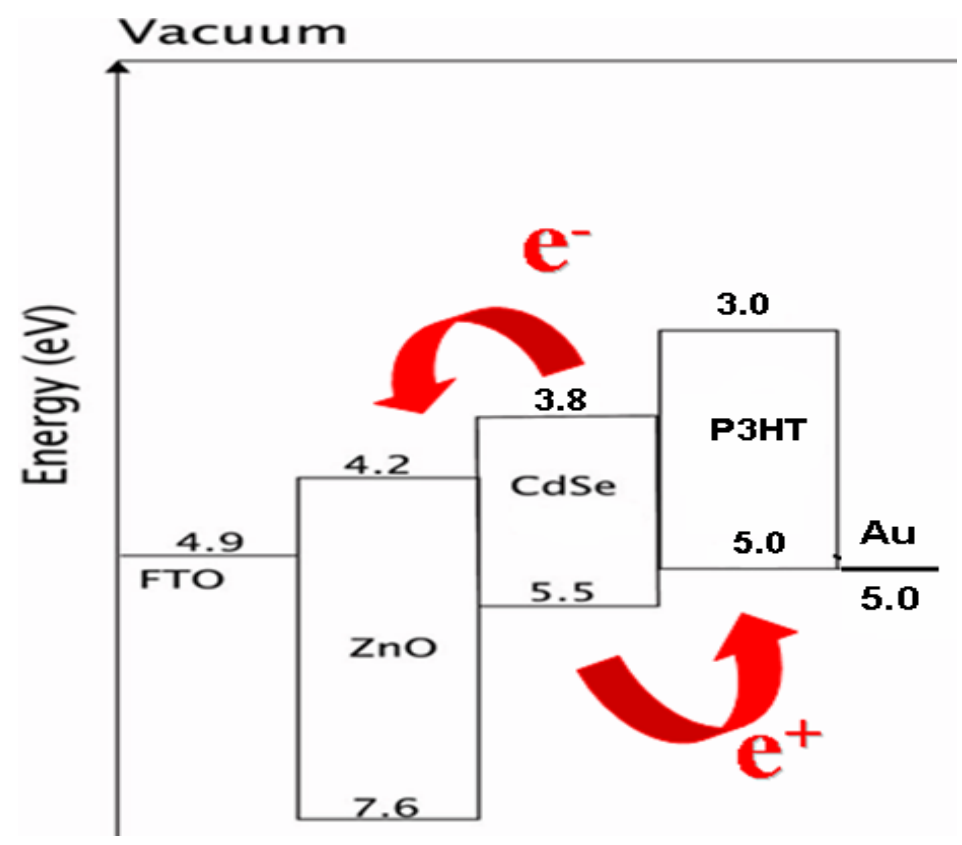

Fig. 48: Schematic band diagram of a nanowire-quantum-dot-polymer heterojunction solar cell 


\subsubsection{Properties of CdSe quantum dot layer}

Figure 49 shows SEM images of $\mathrm{CdSe} / \mathrm{ZnO}$ nanostructures before and after the annealing of $\mathrm{CdSe}$ quantum dots in $\mathrm{CdCl}_{2}$ ambient/air. Figure 49 (a) exhibits the morphology of un-annealed $\mathrm{CdSe}$ quantum dots encapsulating $\mathrm{ZnO}$ nanowires; the deposited un-annealed CdSe quantum-dot layer has an amorphous appearance due to the presence of octadecylamine ligand molecules. The appearance of CdSe quantum dot film after two hours of $\mathrm{CdCl}_{2}$-aided thermal annealing, as shown in Figure 49 (b), demonstrates that structural conversion was activated, resulting in a layer of micron-size interconnected $\mathrm{CdSe}$ particles on the $\mathrm{ZnO}$ nanowires. After annealing for 24 hours, a thin conformal coating of $\mathrm{CdSe}$ forms around the tips of the nanowires, while a spacefilling deposition is obtained near the bottom of the nanowire film, as shown in part (c) of the Figure 49. We can see that the film still consists of vertically-oriented wires even after the deposition of CdSe quantum dots. These images suggest that by the annealing of CdSe quantum dot film coated $\mathrm{ZnO}$ nanowires in $\mathrm{CdCl}_{2}$ / ambient air, one can prepare $\mathrm{CdSe} / \mathrm{ZnO}$ nanostructures with $\mathrm{ZnO}$ cores. 

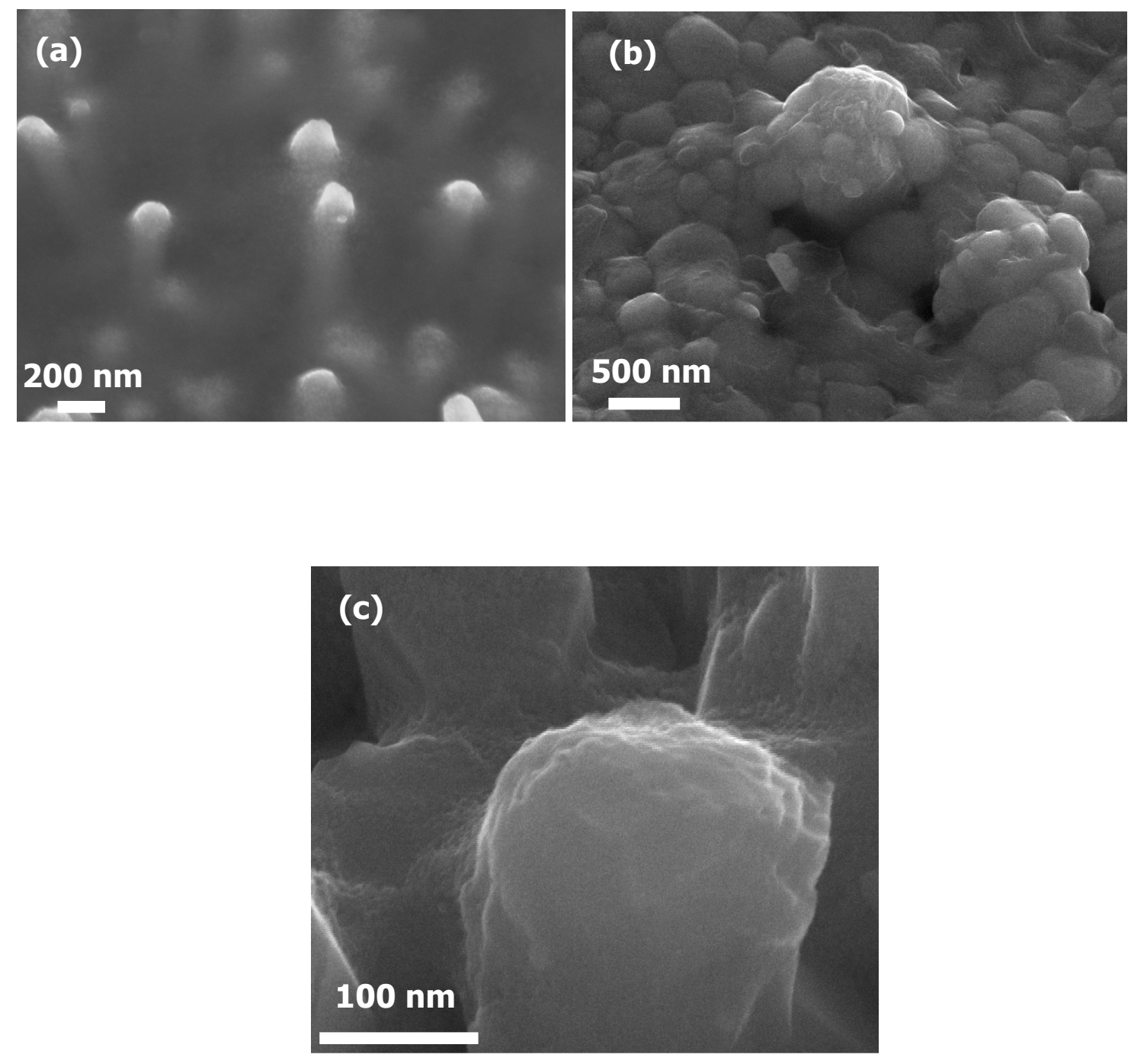

Fig. 49: SEM micrographs showing the CdSe quantum dot film-covered $\mathrm{ZnO}$ nanowires in (a) an as-grown state, and after annealing in $\mathrm{CdCl}_{2}$ for (b) two hours and (c) 24 hours 


\subsubsection{Crystal structure of CdSe quantum-dot film}

The morphology and structural properties of the quantum dot layer and the nanowires before and after annealing were further studied using high-resolution transmission electron microscopy (HRTEM) and energy dispersive x-ray spectroscopy (EDXS). Figures 50 (a) \& (b) show the HRTEM cross-sectional micrographs of CdSe quantum dot film on $\mathrm{ZnO}$ nanowires before and after surfactant-aided thermal annealing process. These images reveal that the annealing processes change the defect concentration in the nanowires and the morphology of the quantum dot layer. Figure 50 (a) illustrates the HRTEM micrograph of un-annealed CdSe quantum-dot film on $\mathrm{ZnO}$, showing the single-crystalline nature of quantum-dots with diameters ranging from 6 to $7 \mathrm{~nm}$. The quantum dot layers change from a loosely connected particle arrangement to a continuous polycrystalline film by surfactant-aided thermal annealing process, as shown in Figure 50 (b). This structural change of the CdSe quantum dot layers caused by annealing considerably enhances the absorption and the transport properties within the CdSe layer, which results in an increased photocurrent response in this p-n junction solar cell structure [212]. It is clear that the as-grown sample has organic ligand molecules surrounding the CdSe quantum dots, blocking the electron injection into the $\mathrm{ZnO}$ and hole injection into the p-type layer. The octadecylamine ligands associated with CdSe quantum dots have a melting point of 120 to $140^{\circ} \mathrm{C}$ and are removed in the annealing process. Energy dispersive X-ray (EDX) spectra confirm that the continuous polycrystalline film consists of CdSe, as shown in Figure 50 (d). The EDX line-scan also reveals that only minimal elemental in-diffusion of Se occurs across the $\mathrm{CdSe} / \mathrm{ZnO}$ 
interface during the formation of the continuous polycrystalline film, whereas the stoichiometry between $\mathrm{Cd}$ and Se is accurately maintained in the case of an un-annealed $\mathrm{CdSe} / \mathrm{ZnO}$ nanostructure (Figure 50 (c)). Moreover, the EDX line profile confirms a reduced coating layer thickness of $\mathrm{CdSe}$ film after the annealing process consistent with the HRTEM results, showing the conversion and compression of the CdSe quantum dots into a conformal and continuous polycrystalline thin film. We also studied the element distributions at the $\mathrm{CdS} / \mathrm{ZnO}$ interface using high resolution energy dispersive X-ray spectroscopy. Figure 50 (e) shows equivalent results for CdS quantum dot film deposited on $\mathrm{ZnO}$ nanowires. It is immediately evident that upon annealing, the $\mathrm{Cd}$ and the $\mathrm{S}$ are distributed homogeneously across the nanowire cross section. Our findings indicate that the presence of sulfur promotes a markedly stronger process of in-diffusion, which is likely due to its larger mobility and reactivity [202]. 

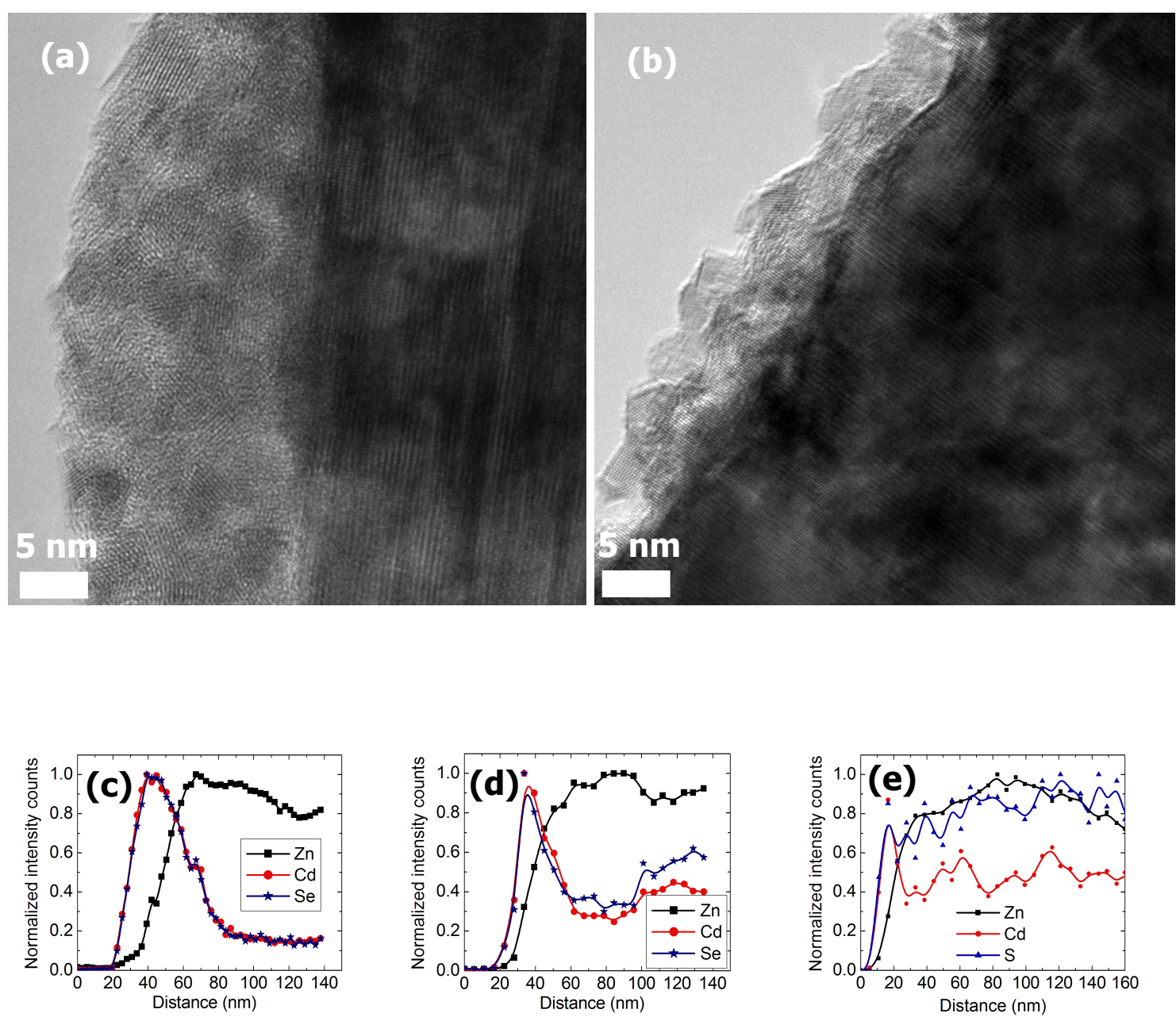

Fig. 50: a) HRTEM micrograph of the $\mathrm{CdSe} / \mathrm{ZnO}$ interface of an as-deposited quantum dot film on a $\mathrm{ZnO}$ nanowire. The image shows that individual quantum dots are singlecrystalline. b) The $\mathrm{CdSe} / \mathrm{ZnO}$ interface of a nanowire from the same sample but annealed for $24 \mathrm{~h}$ at $380^{\circ} \mathrm{C}$. Due to annealing, the CdSe is now-polycrystalline. c) TEMEDX line profile of un-annealed $\mathrm{CdSe} / \mathrm{ZnO}$ nanostructures. d) TEM-EDX line scans across the $\mathrm{CdSe}$-coated $\mathrm{ZnO}$ nanowires after $24 \mathrm{~h}$ anneal at $380^{\circ} \mathrm{C}$. e) TEM-EDX line scans across the $\mathrm{CdS}$-coated $\mathrm{ZnO}$ nanowires after $2 \mathrm{~h}$ anneal at $380^{\circ} \mathrm{C}$ 
4.3.8 Effects of various back contact layers and the formation of $\mathrm{ZnO}$ thin film prior to electrodeposition of $\mathrm{ZnO}$ nanowires

\subsubsection{Electrical measurements}

The photovoltaic properties of the solar cells were characterized by measuring currentvoltage (I-V) curves in the dark and under illumination, through the FTO side, at an intensity of $85 \mathrm{~mW} / \mathrm{cm}^{2}$. Without illumination, all our devices showed diode-like I-V behavior with excellent rectification, as shown in Figure 51. As can be seen in Figure 52, well-defined current-voltage curves were obtained from various solar cell structures under illumination. The short-circuit current improves when we replace the MEH-PPV with a thin P3HT polymer layer. P3HT is known to have a higher hole mobility and higher conductivity, and appears to be more resistant to oxidation [203]. However, the solar cell made using a P3HT back contact exhibits lower shunt resistance than the device made with MEH-PPV as a back contact. In order to resolve this issue, we deposited $\mathrm{ZnO}$ thin film by spray pyrolysis on the FTO substrate prior to $\mathrm{ZnO}$ nanowire electrodeposition, as detailed in section 3.4.1. The formation of this thin film on top of the FTO effectively prevents shunting between the front and back contacts [169, 204]. A remarkably improved fill factor and high photosensitivity were obtained from a solar cell that consisted of a device with the layer sequence FTO/ZnO thin film/NW$\mathrm{ZnO} / \mathrm{CdSe}$ Q-dot film/P3HT/Au. The best device of this type has reached an opencircuit voltage of $0.40 \mathrm{~V}$, a short-circuit current density of $10.0 \mathrm{~mA} / \mathrm{cm}^{2}$, and a conversion efficiency of $2.5 \%$ under illumination at $85 \mathrm{~mW} / \mathrm{cm}^{2}$ with a quartz-halogen 
lamp. In order to achieve long-term stability in these devices, we replaced the polymer back contact with $\mathrm{CuSCN}$, resulting in a device in which all optically active components are inorganic. However, these cells exhibited a relatively low open-circuit voltage and short-circuit current density, as compared to devices made with P3HT or MEH-PPV as a back contact. The formation of cracks in the CuSCN film during deposition and annealing may have contributed to these substandard performances.

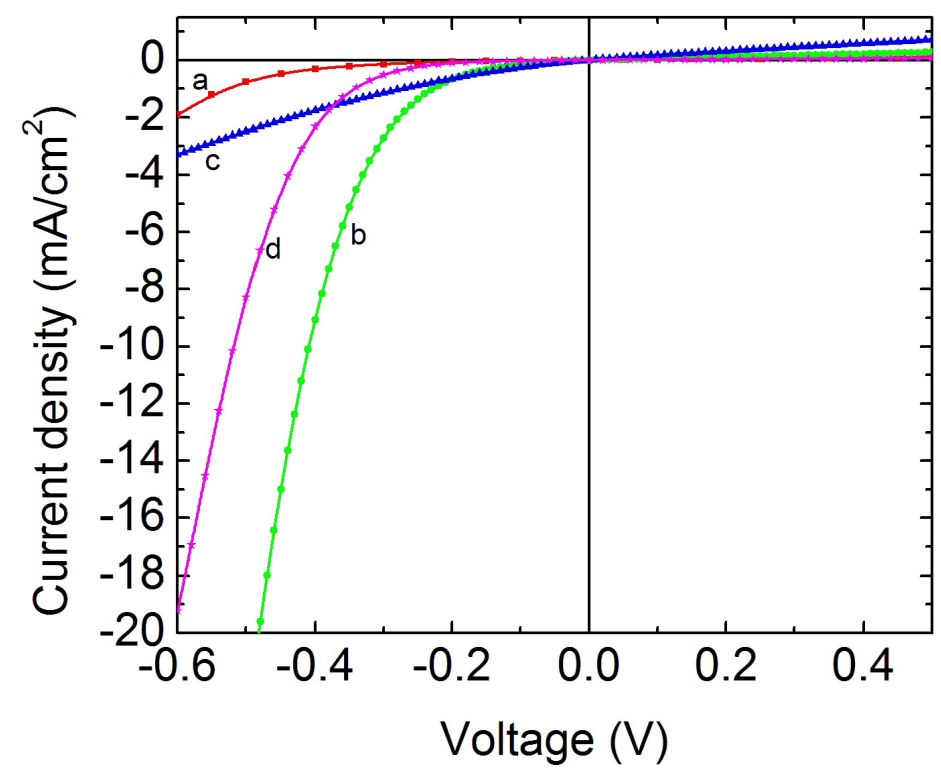

Fig. 51: Dark J-V curves of $\mathrm{ZnO} / \mathrm{CdSe}$ solar cell structure prepared with different $\mathrm{p}$ layer coating: (a) $\mathrm{ZnO} / \mathrm{CdSe} / \mathrm{MEH}-\mathrm{PPV}$, (b) $\mathrm{ZnO} / \mathrm{CdSe} / \mathrm{P} 3 \mathrm{HT}$, (c) $\mathrm{ZnO} / \mathrm{CdSe} / \mathrm{CuSCN}$, and (d) $\mathrm{ZnO}$ thin film/ $\mathrm{ZnO} / \mathrm{CdSe} / \mathrm{P} 3 \mathrm{HT}$ 


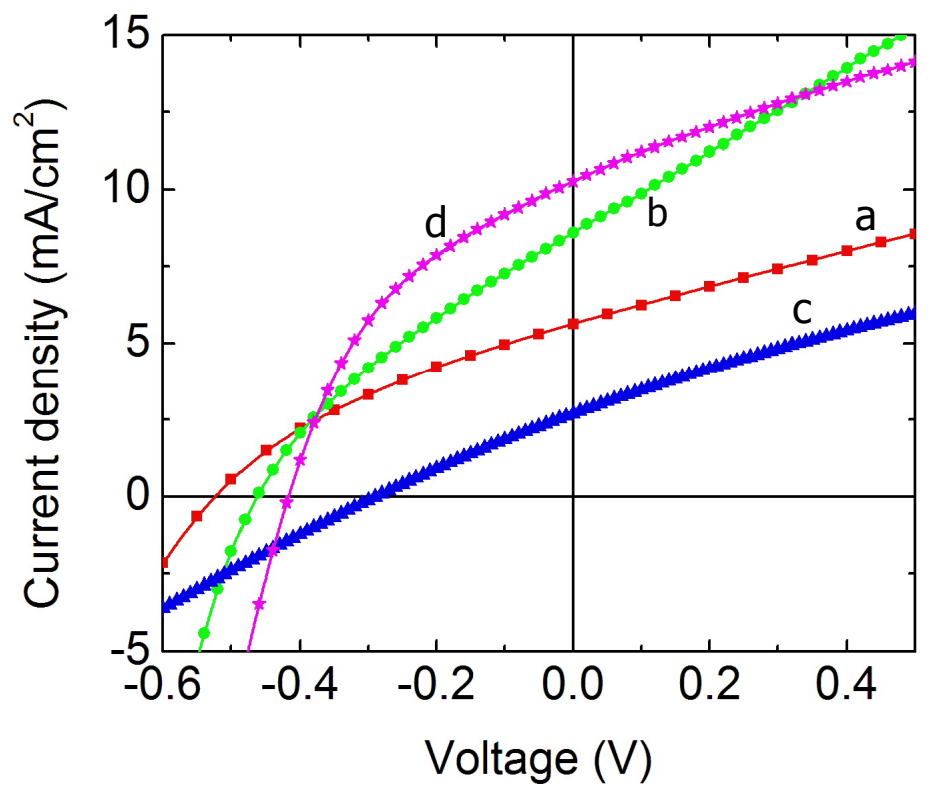

Fig. 52: Light $\mathrm{J}-\mathrm{V}$ curves of $\mathrm{ZnO} / \mathrm{CdSe}$ solar cell structure prepared with different $\mathrm{p}$ layer coating: (a) $\mathrm{ZnO} / \mathrm{CdSe} / \mathrm{MEH}-\mathrm{PPV}$, (b) $\mathrm{ZnO} / \mathrm{CdSe} / \mathrm{P} 3 \mathrm{HT}$, (c) $\mathrm{ZnO} / \mathrm{CdSe} / \mathrm{CuSCN}$, and (d) $\mathrm{ZnO}$ thin film/ZnO/CdSe/P3HT

\subsubsection{Comparative photoresponse measurements}

The spectral dependences of the external quantum efficiencies (EQE) of four solar cells are compared in Figure 53. These cells consist of the following architectures: $\mathrm{ZnO}$ nanowires/CdSe/MEH-PPV, $\mathrm{ZnO}$ nanowires/CdSe/P3HT, $\mathrm{ZnO}$ thin film /ZnO nanowires/CdSe/P3HT, and $\mathrm{ZnO}$ nanowires/CdSe/CuSCN. External quantum efficiency $(\mathrm{EQE})$ is the ratio of the number of electrons measured under short-circuit-current conditions to the number of photons incident on the solar cell at a given wavelength [205]. From the experimental results in this section, we conclude that $\mathrm{CdCl}_{2} /$ ambient air 
annealing produces a CdSe continuous polycrystalline film in which carrier confinement effects are no longer substantial. This promotes the separation and transport of the photogenerated carriers.

We also conclude that incorporating $\mathrm{P} 3 \mathrm{HT}$ into the solar cell (device $\mathrm{ZnO}$ nanowires/CdSe/P3HT) increases the photoresponse in the visible spectral range by a significant amount. By depositing $\mathrm{ZnO}$ thin film at the FTO interface (device: $\mathrm{ZnO}$ thin film/ZnO nanowires/CdSe/P3HT), the photoresponse is further increased, resulting in a maximum external quantum efficiency of $60 \%$ percent in the visible spectral range for this solar cell arrangement. This result reveals that the deposition of $\mathrm{ZnO}$ thin film on FTO plays an important role in improving the performance of the solar cells. We also made solar cells using CuSCN as a back contact instead of p-type polymers. These cells exhibit considerably lower external quantum efficiencies as compared to solar cells made with either a P3HT or MEH-PPV back contact. This decrease in performance could be due to the presence of cracks in the CuSCN film that are formed during its deposition. 


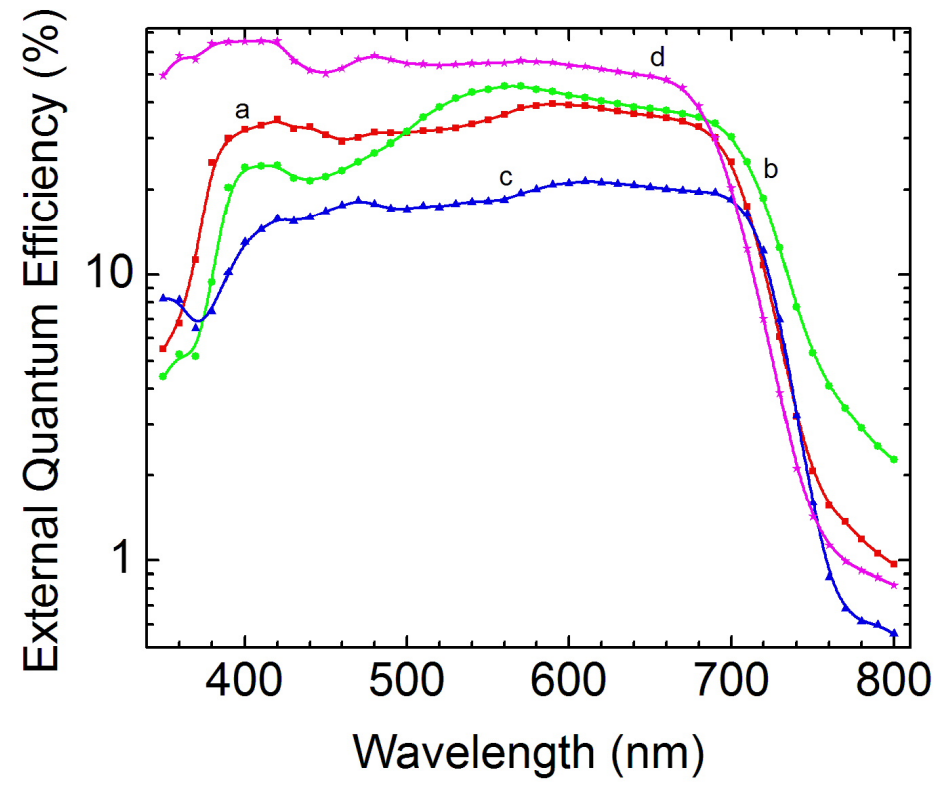

Fig. 53: External quantum efficiency as a function of wavelength for $\mathrm{ZnO} / \mathrm{CdSe}$ nanostructured solar cells prepared with different p-layer coating: (a) $\mathrm{ZnO} / \mathrm{CdSe} / \mathrm{MEH}$ PPV, (b) $\mathrm{ZnO} / \mathrm{CdSe} / \mathrm{P} 3 \mathrm{HT}, \quad$ (c) $\mathrm{ZnO} / \mathrm{CdSe} / \mathrm{CuSCN}$, and (d) $\mathrm{ZnO}$ thin film/ZnO/CdSe/P3HT 
4.3.9 Chemical treatment of the quantum dot with ligand and solvent materials

\subsubsection{Overview}

Here, we illustrate a method of obtaining efficient charge transfer and extraction in a photovoltaic device consisting of a $\mathrm{ZnO}$ nanowire film, $\mathrm{CdSe}$ quantum dots that have undergone solution and ligand exchanges, and a P3HT/Au back contact. These solution and ligand exchanges were carried out in order to form a well-assembled and crack-free thin film of CdSe quantum dots on our $\mathrm{ZnO}$ nanowires. We used a centrifuge technique to replace the initial solution of toluene with pyridine, a solvent which can also play the role of a surfactant by coating the surfaces of the quantum dots and preventing their agglomeration, leading to a more homogeneous deposition of $\mathrm{CdSe}$ quantum dots on the deeply nanostructured $\mathrm{ZnO}$ samples.

We carefully explored the effect of 1,2-ethanedithiol (EDT) treatment on the surface of CdSe quantum dots that are initially capped with octadecylamine for its application in nanowire-quantum-dot solar cells. This solution-phase ligand exchange method, which makes use of EDT as an efficient binding agent, removes the electrically insulating native ligand molecules and enhances the electronic coupling of the $\mathrm{CdSe}$ quantum-dots [206, 207]. In this EDT treatment, the initial ligand molecules that cap the CdSe quantum dots are removed by the dissociative adsorption of EDT on the quantum dot surface, yielding $\mathrm{HS}\left(\mathrm{CH}_{2}\right)_{2} \mathrm{~S}$ - and hydrogen, As a result of octadecylamine removal, the quantum dots move closer together, leading to an enhanced binding between the 
neighboring quantum dots, a binding which is promoted by the sulfur atom in the EDT. Here, we describe the CdSe quantum-dot deposition by a layer-by-layer dip-coating method, which utilizes an efficient linker molecule of EDT. This process improves the electrical and photo-response properties of nanowire-quantum-dot heterojunction solar cells. Such a solution-phase ligand exchange of the CdSe quantum dot provides an energetically favorable charge transfer between the absorber layer and the adjacent layers. This observation suggests that the sulfur atom in EDT is bound efficiently to the quantum dot surface.

Here, we present a three-step deposition process for creating an extremely thin CdSe quantum layer on $\mathrm{ZnO}$ nanowires. This three-step process of CdSe quantum dots involves a pyridine solution exchange followed by an EDT ligand exchange and then a surfactant-aided thermal annealing, resulting in an improved solar cell performance of $3.4 \%$ energy conversion efficiency with an open-circuit voltage of $0.6 \mathrm{~V}$ and a shortcircuit current density of $15 \mathrm{~mA} / \mathrm{cm}^{2}$. This enhanced deposition process led to an increased solar cell performance as compared to our previous solar cells created by drop or spin coated CdSe quantum dots on $\mathrm{ZnO}$ nanowires.

\subsubsection{Appearance of 1,2-ethanedithiol treated CdSe quantum-dot film}

The morphology of the quantum dot layer and the nanowires before and after solution-phase ligand exchange were studied using scanning electron microscopy (SEM). Figure 54 (a) shows the SEM cross-sectional micrograph of the as-synthesized 
CdSe quantum dot film on our $\mathrm{ZnO}$ nanowires prior to thermal annealing of the $\mathrm{CdSe} / \mathrm{ZnO}$ nanostructures. In this state, the CdSe layer appears polymeric rather than poly-crystalline. However, after the EDT-assisted solution-phase ligand exchange, during which the long chain ligand molecules of octadecylamine are removed, the crystalline appearance of the CdSe quantum dot film re-emerges in the SEM crosssectional image, as is displayed in Figure 54 (b). Moreover, annealing in air at $380^{\circ} \mathrm{C}$ in the presence of $\mathrm{CdCl}_{2}$ creates a thin conformal $\mathrm{CdSe}$ coating around the tops of the nanowires whereas a space-filling deposition of $\mathrm{CdSe}$ is obtained near the bottom of the nanowire film, as shown in Figure 54 (c). Vertically oriented nanowires are still clearly visible even after deposition of CdSe quantum dots (Figure 54 (c)). This image suggests that one can prepare $\mathrm{CdSe} / \mathrm{ZnO}$ nanostructures with $\mathrm{ZnO}$ cores by a layer-by-layer dipcoating, employing EDT followed by a surfactant-aided thermal anneal process. We also find that the combination of EDT treatment followed by a CdSe quantum-dot film annealing results in an improved solar cell performance. 

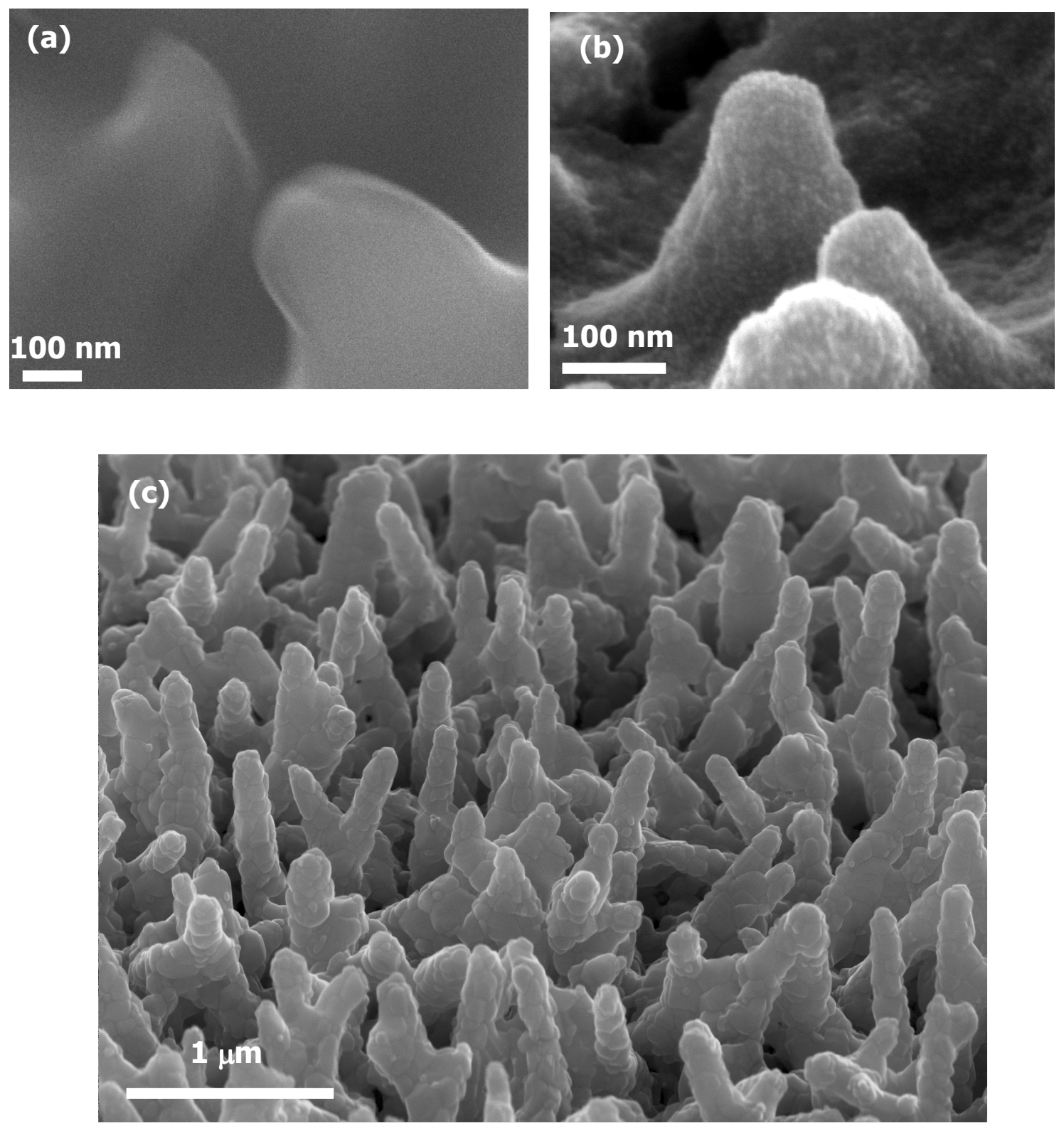

Fig. 54: SEM micrographs showing (a) the $\mathrm{ZnO}$ nanowire film covered with octadecylamine capped CdSe quantum dot film prior to thermal annealing, showing the polymeric nature, (b) the $\mathrm{ZnO}$ nanowire film coated with EDT treated CdSe prior to thermal annealing, and (c) the $\mathrm{ZnO}$ nanowire film coated with EDT treated CdSe after $2 \mathrm{~h}$ annealing 
4.3.9.3 Photovoltaic properties of solar cells made with 1,2-ethanedithiol treated CdSe quantum-dots

The spectral dependence of the external quantum efficiency (EQE) is shown in Figures $55 \& 56$. The un-annealed nanowire-quantum-dot solar cell made with CdSe quantum-dots that have retained their initial ligand molecules (octadecylamine) demonstrates only p-type polymer absorption, as shown in Figure 55. This observation clearly reveals that the long chain length ligand molecules that are initially capped with CdSe quantum dots act as an obstructing layer for electron injection into the $\mathrm{ZnO}$, and most likely also for hole injection into the p-type polymer layer. As we have seen in Figure 55, the photo-response increases noticeably with EDT treatment of the CdSe quantum dots' surface, which causes the photo-response spectrum onset position to shift in energy towards the $7 \mathrm{~nm}$ diameter CdSe quantum dot bandgap value.

In the case of the octadecylamine capped CdSe quantum dots, the linking between the neighboring quantum dot layers occurs via an amine group $\left(\mathrm{NH}_{2}\right)$ whereas thiol $(\mathrm{SH})$ is responsible for this linking in the EDT-treated quantum-dots. The separation between the two quantum layers is roughly $4-5 \mathrm{~nm}$ in the untreated state, and becomes even smaller, around $0.8 \mathrm{~nm}$, after the EDT treatment [208]. Therefore, we can conclude that the nanoparticle film made by EDT-treated layer-by-layer dip-coating strongly improves the electronic coupling of nanoparticles by removing the electrically insulating ligand molecules that were originally capping the CdSe quantum-dots. 


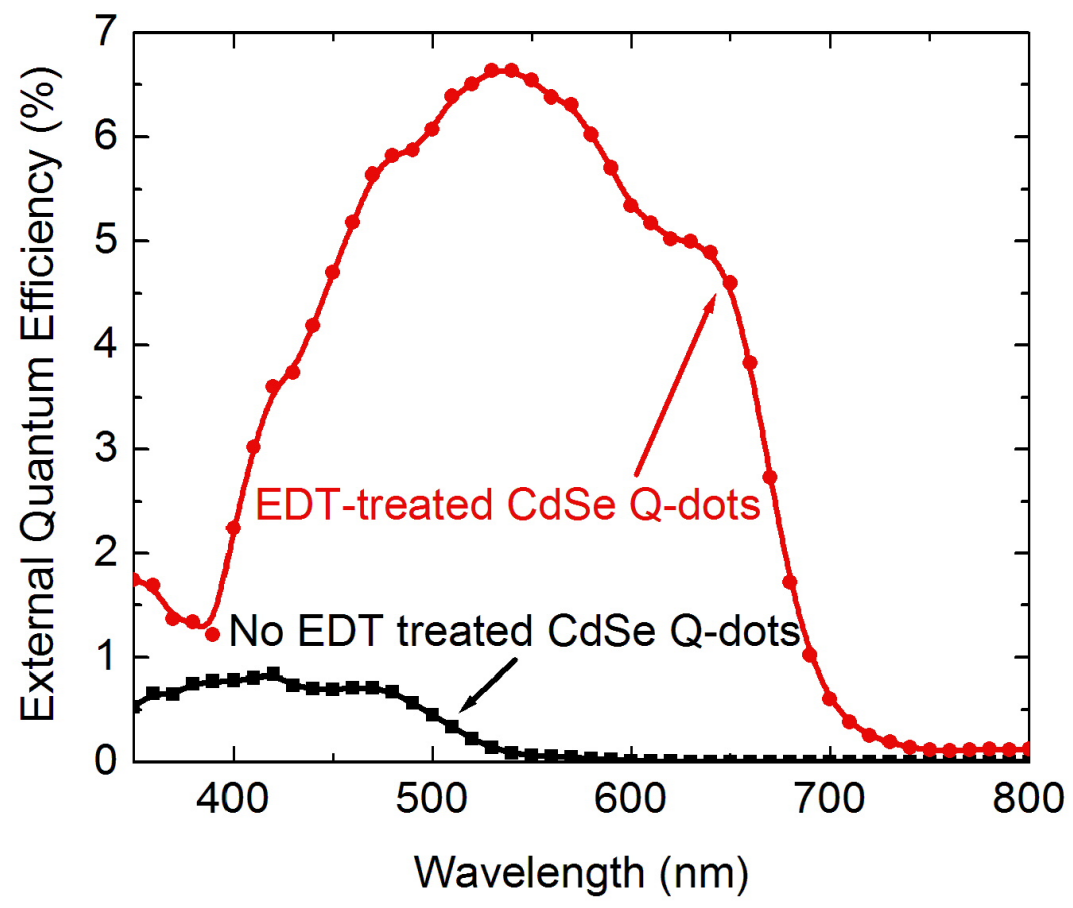

Fig. 55: External quantum efficiency as a function of wavelength for un-annealed $\mathrm{ZnO}$ thin film/ZnO/CdSe/P3HT nanostructured solar cells

Figure 56 shows the photo-response of solar cells after surfactant-aided thermal annealing, with one group of cells using EDT treated quantum dots and another group of cells using quantum dots not treated with EDT. We can also see that the CdSe quantum dots treated with EDT followed by annealing in $\mathrm{CdCl}_{2}$ ambient air further increases the quantum efficiency, as shown in Figure 56. The annealing produces a continuous polycrystalline film of $\mathrm{CdSe}$ in which carrier confinement effects are no longer active. The formation of the polycrystalline film efficiently promotes the separation of photo-generated carriers. The photo-response spectra also show that the onset position moves further towards the CdSe bulk bandgap energy due to the loss of 
spatial confinement during the thermal annealing in $\mathrm{CdCl}_{2} /$ ambient air. The combination of layer-by-layer dip-coating with EDT as binding agent, and the surfactant-aided thermal annealing, play an important role in improving the performance of solar cells, resulting in a maximum external quantum efficiency of $40 \%$ in the visible spectral range.

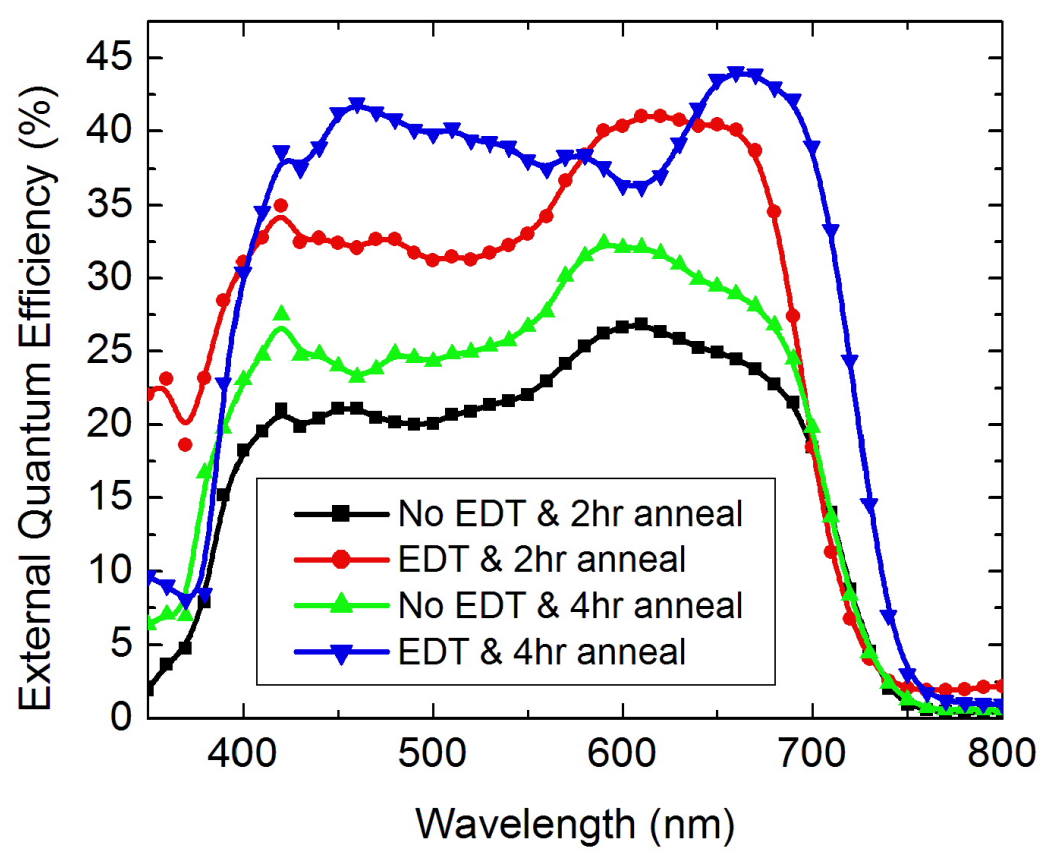

Fig. 56: External quantum efficiency as a function of wavelength for $380^{\circ} \mathrm{C}$ annealed nanowire-quantum-dot solar cells 
4.3.9.4 Photoresponse measurements: The effect of pyridine solution exchange

Figure 57 compares two solar cells, one cell prepared from CdSe quantum-dots suspended in toluene, the other made with CdSe quantum-dots that have undergone pyridine solvent exchange. The external quantum efficiency profile of a solar cell made with $\mathrm{CdSe}$ quantum-dots dispersed in toluene, and deposited on the $\mathrm{ZnO}$ nanowires through a layer-by-layer dip-coating process utilizing EDT as a linker molecule, is shown by the black curve. The red curve represents the external quantum efficiency plot of a solar cell constructed with CdSe quantum-dots suspended in pyridine and deposited using the same EDT-assisted layer-by-layer method. Solar cells made of CdSe quantum dots that have been chemically treated with pyridine solvent and EDT ligands exhibit an improved external quantum efficiency of above $70 \%$ at a wavelength around $700 \mathrm{~nm}$. This observed improvement is likely due to a more crack-free film surface (See Figure 54 (c)). 


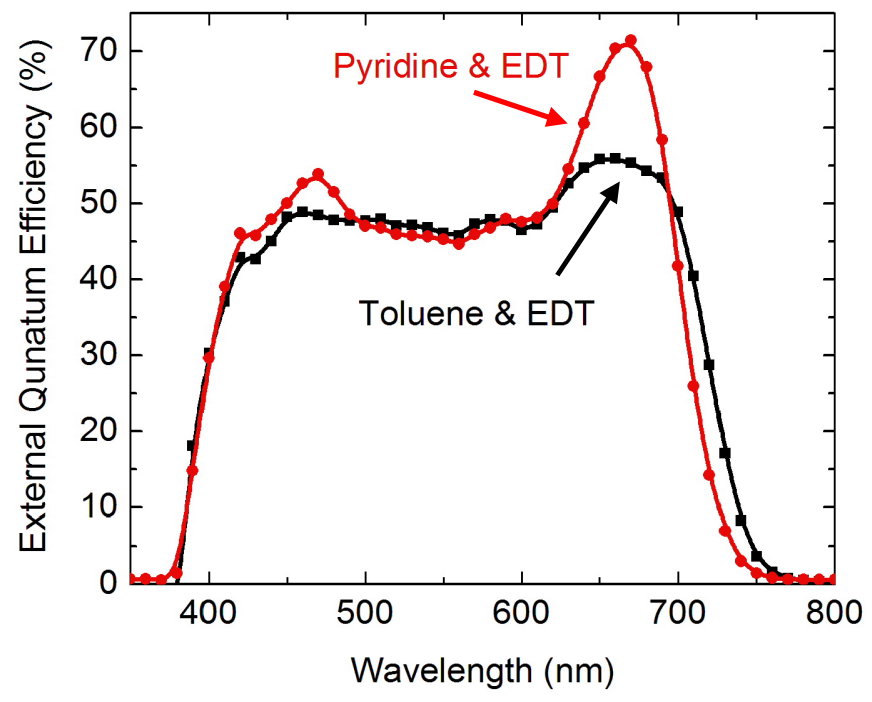

Fig. 57: External quantum efficiency as a function of wavelength for $380^{\circ} \mathrm{C}$ annealed nanowire-quantum-dot solar cells prepared with CdSe quantum dots that have undergone pyridine solution and EDT ligand exchange (shown by red curve) and CdSe quantum dots that are suspended in Toluene and caped with EDT ligand molecules (shown by black curve)

4.3.9.5 Electrical measurements: Improved transport with pyridine and 1,2-ethanedithiol treatment in the preparation of nanowire-quantum-dot solar cells

The current-voltage profiles in dark and light conditions of our best solar cell prepared with CdSe quantum dots dispersed in pyridine and capped with EDT are shown in Figure 58. The dark current-voltage curve shows a well-defined diode characteristic with an excellent rectifying behavior. The light current-voltage curve 
exhibits a very good photovoltaic performance with an open circuit-voltage of $0.6 \mathrm{~V}$, a short-circuit current of $15 \mathrm{~mA} / \mathrm{cm}^{2}$, and an energy conversion efficiency of $3.4 \%$. This efficiency is one of the best to have been achieved for this type of device to date. The combination of pyridine solution exchange, EDT ligand exchange, and the surfactantaided thermal annealing of the CdSe quantum dots are the major factors responsible for improving the efficiency of our nanowire-quantum-dot-polymer solar cells. These devices establish an excellent starting point for the development of very low cost solar cells.

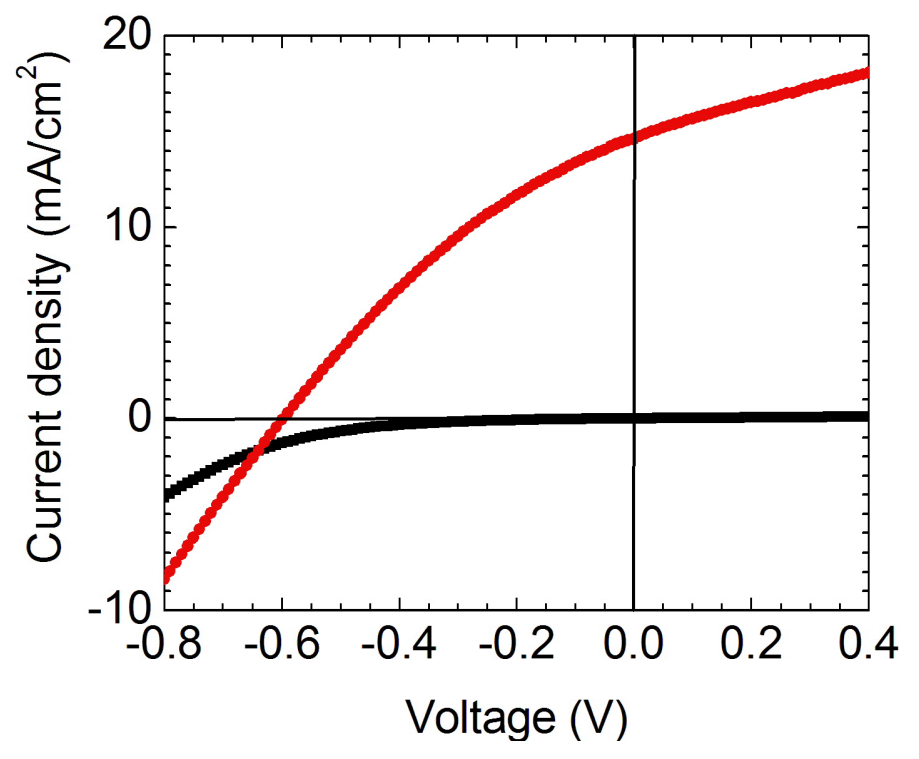

Fig. 58: Current-voltage characteristics under dark and $85 \mathrm{~mW} / \mathrm{cm}^{2}$ illumination conditions for a nanowire-quantum-dot solar cell prepared with CdSe quantum-dots that have undergone pyridine solution and EDT ligand exchange 


\subsubsection{The effect of ageing on photovoltaic performance}

The stability of our best performing solar cell is shown in Figure 59. This cell was composed of CdSe quantum dots that had been suspended in pyridine solution and capped with EDT. The front contact was comprised of a $\mathrm{ZnO}$ thin film deposited on an FTO substrate. A P3HT-Au layer was used as the back contact. Figure 59 shows the external quantum efficiency data as a function of wavelength over a three month period of testing the device in ambient air, at room-temperature conditions, and without encapsulation. This plot indicates that the efficiency of the device increases significantly in the first three days, and then retains a stable performance for nearly four weeks. This four week period is followed by a gradual decrease in efficiency accompanied by an observable decrease in the performance of the cell. The initial three days of increasing device efficiency could be attributed to the surface passivation of the back contact, which occurs naturally, without the addition of any surfactants, following deposition of this contact. Once this improvement is achieved, the solar cell shows stable performance for several weeks due to the stability of P3HT against oxidation. Other solar cells that were made of either MEH-PVV, PTB7, or PCDTBT as a p-type back contact layer instead of P3HT did not exhibit this same month-long stability, all showing a decrease in efficiency within one week of inception. However, the cells made with P3HT demonstrate only a slight decrease in efficiency after one month of stable performance. This decrease is likely caused by the eventual oxidation of the P3HT layer, a hypothesis which was tested and confirmed in solar cells that were encapsulated with 
epoxy. These solar cells indeed degraded at a slower rate than those devices made without encapsulation.

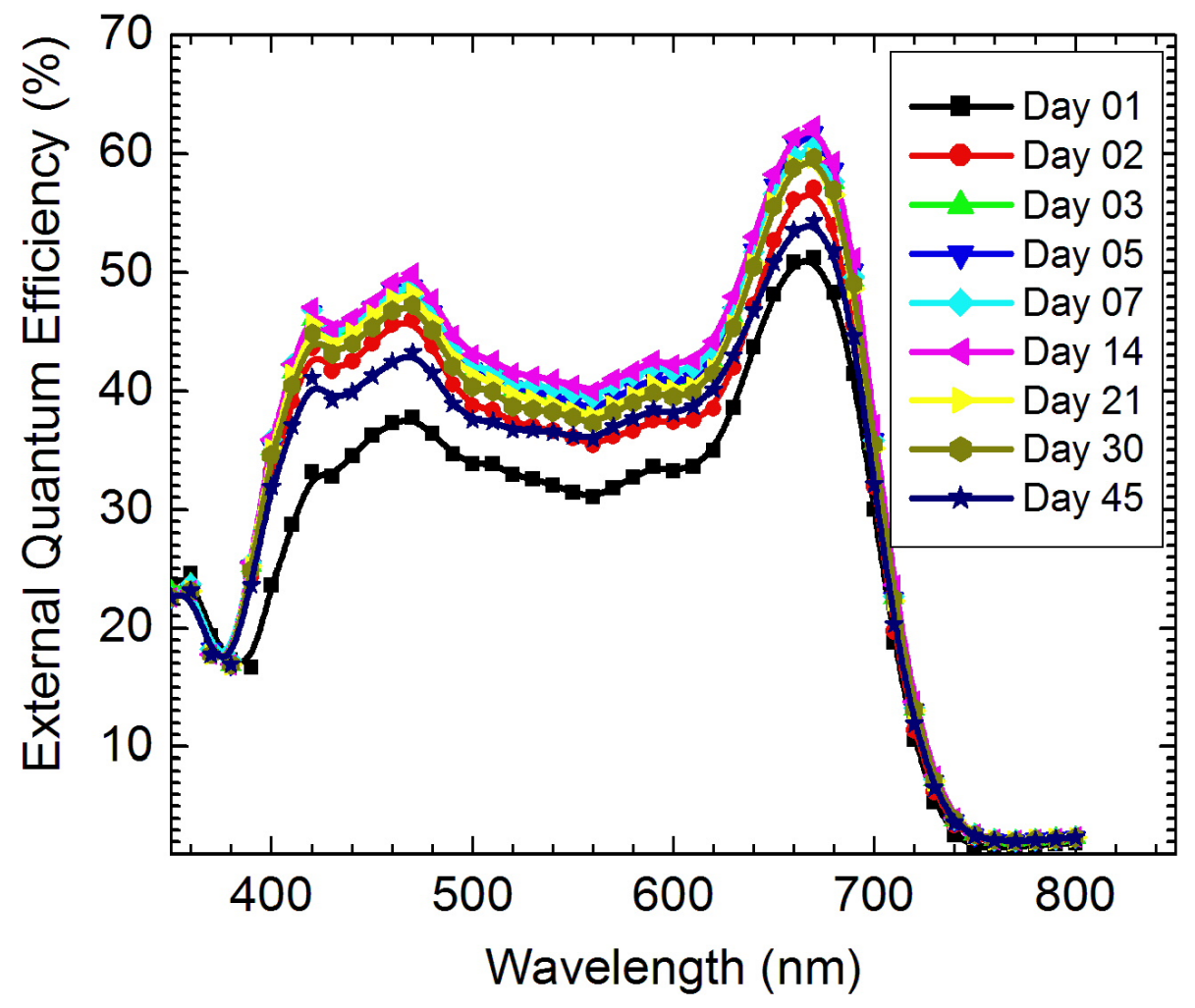

Fig. 59: External quantum efficiency plots as a function of wavelength for a solar cell that has been kept in ambient air conditions at room-temperature for various periods of time 


\section{Conclusion}

We have studied fundamental properties and device applications of nanoscale particles, such as nanowires and quantum dots. Many of these devices also used polymers as insulating or contacting agents. $\mathrm{ZnO}$ was used as the model nanowire material and these $\mathrm{ZnO}$ nanowires were synthesized at low temperatures by electrodepostion. The unique properties of $\mathrm{ZnO}$ and the solution-based synthesis of $\mathrm{ZnO}$ nanostructures make this material extremely attractive for its use in device applications. Any efficient device application would require effective doping of these nanowires, a process which is known to modify their electronic structure and thus their optical and electrical properties. We explored the feasibility of $\mathrm{ZnO}$ doping, and established useful routines of post-deposition processing. We found that $\mathrm{ZnO}$ nanowires can be conveniently doped by the solution phase with a variety of impurities. SIMS

measurements and TEM-EDX line profiles confirmed impurity incorporation and doping in the bulk of the nanowires. These wires have surprisingly high concentrations of lattice distortions and strain related areas; however, annealing at moderate temperatures creates strain relaxation by the formation of dislocation-type defects. As a result, in the as-grown state, the doping has only very limited effects on these as-grown $\mathrm{ZnO}$ nanowires' optical properties. After annealing at $380^{\circ} \mathrm{C}$ the impurity-related effects become clearly visible, hence, luminescence efficiency is increased. Al doping, in particular, can enhance the luminescence efficiency drastically in the blue and ultraviolet spectral regions. At room temperature, PL quantum efficiencies of 16 percent 
can be reached in Al doped films. Other impurities led to spectral shifts in the visible part of the electroluminescence spectra after annealing.

We demonstrated a vertical nanowire-based light-emitting diode (LED) consisting of a p-type polymer of PEDOT:PSS and n-type $\mathrm{ZnO}$ nanowires on an FTO/glass substrate. At room-temperature, our vertical nanowire/polymer LED structure exhibited a broad emission band in the visible region due to the distributions of different defect states, and a narrow UV emission peak caused by excitonic transitions and centered at $393 \mathrm{~nm}$. We also converted this planar LED into a flexible LED by replacing FTO/glass with ITO/PET film, obtaining similar electroluminescence from this flexible LED arrangement as that achieved from a planar LED arrangement. These LED structures also show that their electroluminescence is qualitatively similar to the photoluminescence obtained from $\mathrm{ZnO}$ nanowires. Transport limitations such as longer transport path length and higher tunneling barriers at the injection contacts cause a slight reduction in the blue and ultra-violet contributions in these LED structures as compared to those from the photoluminescence. Due to the enormous amount of research in this area, it can be expected that more sophisticated LED structures with the ability to overcome these limitations will be constructed in the near future. Thus, $\mathrm{ZnO}$ nanowires grown at low temperatures will soon open the door for a broad field of applications in optical devices on glass, plastics, $\mathrm{Si}$ wafers, and other sensitive substrates. We also observed superparamagnetic behavior in $\mathrm{Mn}, \mathrm{Co}$, and $\mathrm{Cu}$ doped $\mathrm{ZnO}$ nanowire samples at room temperature. $\mathrm{Al}$ doped and undoped $\mathrm{ZnO}$ nanowires were used as references and gave no visible magnetic response. The 
superparamagnetism in $\mathrm{ZnO}$ nanowires could extend their use to applications in the biolabeling and bio-imaging fields.

Another development of our research was the creation of an innovative $\mathrm{ZnO}$ nanowire-based heterojunction solar cell achieved through the incorporation of a dispersion of CdSe quantum dots. The $\mathrm{ZnO}$ nanowires were used to provide a singlegrain path from the absorber layer to the front contact. Our results indicate that the surface area enhancement provided by the $\mathrm{ZnO}$ nanowires improves charge transfer when these nanowires are incorporated with materials that exhibit favorable band energy offsets. The deposited CdSe quantum dots were converted into a continuous polycrystalline thin film with excellent surface conformality upon annealing. The back contact was provided by a p-type material/metal contact. We explored the use of MEHPPV and P3HT, as well as CuSCN, as a p-type material for this contact. Furthermore, our recent $\mathrm{ZnO}$ nanowire-based heterojunction solar cells have been prepared from CdSe quantum-dots dispersed in pyridine. These quantum dots also utilize 1.2ethanedithiol (EDT) as a binding agent to remove the initial electrically-insulating capping ligand molecule and enhance their electronic coupling. The dispersion of $\mathrm{CdSe}$ quantum dots in pyridine provides a crack-free and well assembled CdSe quantum dot film covering the $\mathrm{ZnO}$ nanowires. The formation of this homogeneous film can be attributed to the naturally slower evaporation of pyridine at room-temperature as compare to toluene, the solution in which these CdSe quantum dots are initially suspended. The combination of the pyridine solution and EDT ligand exchange of CdSe quantum-dots followed by $\mathrm{CdCl}_{2}$-aided thermal annealing produces an excellent 
photogenerated carrier transfer between neighboring layers, thereby increasing the solar cell's quantum efficiency. Our best photo-conversion efficiency was 3.4 percent and was obtained through the use of a solution and ligand exchange of CdSe quantum dots, well-annealed $\mathrm{CdSe}$ absorber, P3HT/Au back contact, and FTO/ZnO thin film/ZnO nanowire front contact. These devices represent a good starting point for the development of very cost efficient nanostructured solar cells. With further refinement of both the CdSe quantum dots' deposition processes and their surface treatments by chemical methods, it appears likely that photo-response will soon be achieved in flexible nanowire-quantum-dot devices in which conventional CdSe thermal annealing procedures cannot be performed. 


\section{References}

[1] S. Chattopadhyay, L. C. Chen, and K. H. Chen, NPG Asia Mater., 3, 74, 2011

[2] H. Hirayama, N. Noguchi, S. Fujikawa, J. Norimatsu, N. Kamata, T. Takano, and K. Tsubaki, Proc. SPIE, 7216, 721621, 2009

[3] H. A. Atwater and A. Polman, Nat. Mater., 9, 205, 2010

[4] A. Akpinar, F. Tavşan, M. İ. Kömürcü, M. H. Filiz, and K. Kaygusuz, JournalTOCs, 7 (1), 28, 2012

[5] H. J. Round, Electrical World, 19, 309, 1907

[6] R. D. Dupuis and M. R. Krames, J. Lightw. Technol., 26, 1154, 2008

[7] S. Nakamura, M. Senoh, and T. Mukai, Jpn. J. Appl. Phys., 32, L8, 1993

[8] S. Nakamura, M. Senoh, and T. Mukai, Appl. Phys. Lett., 62, 2390, 1993

[9] S. Nakamura, M. Senoh, M. Nagahama, N. Iwasa, T. Yamada, T. Matsushita, Y.Sugimoto, and H. Kiyoku, Appl. Phys. Lett., 69, 4056, 1996

[10] M. A. Zimmler, T. Voss, C. Ronning, and F. Capasso, Appl. Phys. Lett., 94, 241120, 2009

[11] O. Lupan, T. Pauporte, and B. Viana, Adv. Mater., 22, 3298, 2010

[12] R. Könenkamp, R.C. Word, C. Schlegel, Appl. Phys. Lett., 85, 6004, 2004

[13] R. Köenkamp, R.C. Word, M. Godinez, Nano Lett., 2005, 5, 2005

[14] B. Seipel, A. Nadarajah, B. Wutzke, and R. Könenkamp, Mater. Lett., 63, 736, 2009

[15] A. Nadarajah, R. C. Word, J. Meiss, and R. Könenkamp, Nano Lett., 8, 534, 2008

[16] A. Shah, P. Torres, T. Tscharner, N. Wyrsch, and H. Keppner, Science, 285, 692, 1999

[17] O. Shevaleevskiy, Pure Appl. Chem., 80, 2079, 2008

[18] D. M. Chapin, C. S. Fuller, and G. L. Pearson, J. Appl. Phys. 25, 676, 1954

[19] W. Shockley and H. J. Queisser, J. Appl. Phys., 32, 510, 1961 
[20] M. A. Green, K. Emery, Y. Hishikawa, and W. Warta, Prog. Photovolt: Res. Appl. $19,84,2011$

[21] L. Dou, J. You, J. Yang, C.-C. Chen, Y. He, S. Murase, T. Moriarty, K. Emery, G. Li, and Y. Yang, Nature Photonics, 6, 180, 2012

[22] B. O'Regan and M. Grätzel, Nature, 353, 737, 1991

[23] A. Nadarajah, R. C. Word, K. VanSant, and R. Könenkamp, Phys. Stat. Sol. (b), $245,1834,2008$

[24] W. Van Roosbrozck and W. Shockley, Phys. Rev., 94, 1558, 1954

[25] P. Würfel, J. Phys. C. Solid State Phys., 15, 3967, 1982

[26] D. M. Bagnall, Y. F. Chen, Z. Zhu, T. Yao, S. Koyama. M. Y. Shen, and T. Goto, Appl. Phys. Lett., 70, 2230, 1997

[27] M. H. Huang, S. Mao, H. N. Feick, H. Q. Yan, Y. Y. Wu, H. Kind, E. Weber, R. Russo, and P. D. Yang, Science, 292, 1897, 2001

[28] C. Levy-Clement, R. Tena-Zaera, M. A. Ryan, A. Katty, and G. Hodes, Adv. Mater., 17, 1512, 2005

[29] Ü. Özgür, Ya. I. Alivov, C. Liu, A. Teke, M. A. Reshchikov, S. Doğan, V. Avrutin, S. J. Cho, and H. Morkoç, J. Appl. Phys., 98, 041301, 2005

[30] J. H. Lim, C. K. Kang, K. K. Kim, I. K. Park, D. K. Hwang, and S. J. Park, Adv. Mater., 18, 2720, 2006

[31] Y. J. Zeng, Z. Z. Ye, W. Z. Xu, D. Y. Li, J. G. Lu, L. P. Zhu, and B. H. Zhao, Appl. Phys. Lett., 88, 062107, 2006

[32] Y. Cao, L. Miao, S. Tanemura, M. Tanemura, Y. Kuno, and Y. Hayashi, Appl. Phys. Lett., 88, 251116, 2006

[33] L. Dunlop, A. Kursumovic, and J. L. Macmanus-Driscoll, Appl. Phys. Lett., 93, 172111, 2008

[34] D. K. Hwang, S. H. Kang, J. H. Lim, E. J. Yang, J. Y. Oh, J. H. Yang, and S. J. Park, Appl. Phys. Lett., 86, 222101, 2005

[35] G. D. Yuan, W. J. Zhang, J. S. Jie, X. Fan, J. A. Zapien, Y. H. Leung, L. B. Luo, P. F. Wang, C. S. Lee, and S. T. Lee, Nano Lett., 8, 2591, 2008 
[36] B. Xiang, P. Wang, X. Zhang, S. A. Dayeh, D. P. R. Aplin, C. Soci, D. Yu, and D. Wang, Nano Lett., 7, 323, 2007

[37] Y. H. Leung, Z. B. He, L. B. Luo, C. H. A. Tsang, N. B. Wong, W. J. Zhang, and S. T. Leeet, Appl. Phys. Lett., 96, 053102, 2010

[38] Y. Yang, X. W. Sun, B. K. Tay, G. F. You, S. T. Tan, and K. L. Teo, Appl. Phys. Lett., 93, 253107, 2008

[39] http://www.webelements.com/compounds/zinc/zinc_oxide.html

[40] J. E. Jaffe, J. A. Snyder, Z. Lin, and A. C. Hess, Phys. Rev., B 62, 1660, 2000

[41] J. R. Chelikowsky, Sol. Stat. Comm., 22, 351, 1977

[42] U. Rössler, Phys. Rev., 184, 733, 1969

[43] S. Bloom and I. Ortenburger, Phys. Stat. Sol. (b), 58: 561, 1973

[44] M. Usuda, N. Hamada, T. Kotani, and M. van Schilfgaarde, Phys. Rev., B 66, 125 101,2002

[45] I. Ivanov and J. Pollmann, Phys. Rev., B 24,7275, 1981

[46] D. Vogel, P. Krüger, and J. Pollmann, 316, 1995

[47] F. J. Himpsel, Adv. Phys., 32, 1, 1983

[48] T.G. Pedersen, Phys Status Solidi (c), 2 (12), pp. 4026, 2005

[49] C.J. Lan, H.Y. Cheng, R.J. Chung, K.F. Kao, and T.S. Chin, J. Electrochem. Soc., 154 (3), D117, 2007

[50] J.B. Cui and U.J. Gibson, Appl Phys Lett., 87, 133108, 2005

[51] B. B. Lipinski, D. H. Mosca, N. Mattoso, W. H. Schreiner, and A. J. A. de Oliveira, Electrochem. Solid State Lett., 7 (10), C115, 2004

[52] M. Kemell, F. Dartigues, M. Ritala and M. Leskelä, Thin Solid Films, 434, 20, 2003

[53] G. Machado, D. N. Guerra, D. Leinen, J. R. Ramos-Barrado, and R. E. Marotti, Thin Solid Films, 490, 124, 2005

[54] H. Ishizaki, M. Izaki, and T. Ito, J Electrochem. Soc., 148, C540, 2001 
[55] J. B. Cui and U. J. Gibson, Appl. Phys. Lett., 87, 133108, 2005

[56] J. B. Cui and Q. Zeng, J. Appl. Phys., 99, 08M113, 2006

[57] J. B. Cui and U. J. Gibson, Phys. Rev B 74, 045416, 2006

[58] A. Janotti and Chris G Van de Walle, Rep. Prog. Phys., 72, 126501, 2009

[59] M. D. McCluskey and S. J. Jokela, J. Appl. Phys., 106, 071101, 2009

[60] B. C. Cavenett, Advances in Physics, 30, 475, 1981

[61] F. Yang, T. He, J. B. Chen, and F. Pana, J. Appl. Phys., 91, 3114, 2002

[62] Chih-Chang Chen, Yung-Jung Hsu, Yi-Feng Lin, and Shih-Yuan Lu, J. Phys. Chem. C, 112, 17964, 2008

[63] B. Y. Geng, T. Xie, T., X. S. Peng, Y. Lin, X. Y. Yuan, G. W. Meng, and L. D. Zhang, Appl. Phys. A: Mater. Sci. Process., 77, 363, 2003

[64]. I. Park, Y. H. Jun, S. W. Jung, and G. C. Yi, Appl. Phys. Lett., 82, 964, 2003

[65] S. Peulon and D. Lincot, J. Electrochem. Soc., 145, 864, 1998

[66] R. Könenkamp, K. Boedecker, M. C. Lux-Steiner, M. Poschenrieder, F. Zenia, and C. Leavy-Clement, Appl. Phys. Lett., 77, 2575, 2000

[67] L. Vayssieres, Adv. Mater., 15, 464, 2003

[68] S. Peulon and D. Lincot, Adv. Mater., 8, 166, 1996

[69] M. Izaki and T. Omi, Appl. Phys. Lett., 68, 2439, 1996

[70] Y. B. Li, Y. Bando, T. Sato, and K. Kurashima, Appl. Phys. Lett., 81, 144, 2002

[71] X. Y. Kong and Z. L. Wang, Nano Lett., 3, 1625, 2003

[72] W. L. Hughes and Z. L. Wang, Appl. Phys. Lett., 86, 043106, 2005

[73] H. Gao, X. T. Zhang, M. Y. Zhou, Z. G. Zhang, X. Z. Wang, Nanotechnology, 18, 065601, 2007

[74] A. Osinsky and S. Karpov, in Zinc Oxide Bulk, Thin Films and Nanostructures: Processing, Properties and Applications, edited by C. Jagadish and S. J. Pearton (Elsevier, London), 525, 2006 
[75] W. Prellier, A. Fouchet, B. Mercey, Ch. Simon, and B. Raveau, Appl. Phys. Lett., 82, 3490, 2003

[76] Hyeon-Jun Lee and Se-Young Jeong, Appl. Phys. Lett., 81, 4020, 2002

[77] Xue-Chao Liu, Er-Wei Shi, Zhi-Zhan Chen, Hua-Wei Zhang, Li-Xin Song, Huan Wang and Shu-De Yao, J. Cryst. Growth, 296, 135, 2006

[78] D. B. Buchholz, R. P. H. Chang, J. H. Song and J. B. Ketterson, Appl. Phys. Lett., 87, 082504, 2005

[79] M. A. Thomas and J. B. Cui, J. Phys. Chem. Lett., 1, 1090, 2010

[80] Z. L. Wang, Mater. Today, 7 (6), 26, 2004

[81] A. Nadarajah, R. C. Word, J. Meiss, and R. Könenkamp, Nano Lett., 8, 534, 2008

[82] R. Könenkamp, R. C. Word, M. Dosmailov, J. Meiss, and A. Nadarajah, J. Appl. Phys., 102, 056103, 2007

[83] A. Nadarajah and R. Könenkamp, Nanotechnology, submitted, 2011

[84] B. Seipel, A. Nadarajah, B. Wutzke, and R. Könenkamp, Mater. Lett., 63, 736, 2009

[85] A. Nadarajah and R. Könenkamp, Phys. Status Solidi B, 248 (2), 334, 2011

[86] A. Nadarajah, R. C. Word, K. VanSant, and R. Könenkamp, Phys. Stat. Sol. (b), $245,1834,2008$

[87] A. Nadarajah, R. C. Word, and R. Könenkamp, Mater. Res. Soc. Symp. Proc., 1178,2009

[88] L. A. Kolodziejski, R. L. Gunshor, and A. V. Nurmikko, Annu. Rev. Mater. Sci. 25, 7111995, 1997

[89] A. Nurmikko and R. Gunshor, Semicond. Sci. Technol., 12, 1337, 1997

[90] S. Nakamura, T. Mukai, and M. Senoh, Appl. Phys. Lett., 64, 1687, 1994

[91] S. Nakamura. J. Vac. Sci. Technol., A 13, 705, 1995

[92] T. Nishida, T. Ban, and N. Kobayashi, Appl. Phys. Lett., 82, 1, 2003

[93] M. Iwaya, S. Takanami, A. Miyazaki, Y. Watanabe, S. Kamiyama, H. Amano, and I. Akasaki, Jpn. J. Appl. Phys., 42, 400, 2003 
[94] J. P. Zhang, A. Chitnis, V. Adivarahan, S. Wu, V. Mandavilli, R. Pachipulusu, M. Shatalov, G. Simin, J.W. Yang, and M. Asif Khan. Appl. Phys. Lett., 81, 4910, 2002

[95] O. Ambacher, J. Phys., D 31, 2653, 1998

[96] W. R. L. Lambrecht, S. Limpijumnong, and B. Segall, MRS Internet J. Nitride Semicond. Res., 4S1, G6.8, 1995

[97] K. Shindo, A. Morita, and H. Kamimura, J. Phys. Soc. Jpn., 20, 11, 2054, 1995

[98] I. T. Drapak, Semiconductors 2, 624, 1968

[99] A. E. Tsurkan, N. D. Fedotova, L. V. Kicherman, and P. G. Pas'ko, Semicond. Semimetals, 6, 1183, 1975

[100] H. Ohta, K. Kawamura, M. Orita, M. Hirano, N. Sarukura, and H. Hosono, Appl. Phys. Lett., 77, 475, 2000

[101] Y. I. Alivov, J. E. Van Nostrand, D. C. Look, M. V. Chukichev, and B. M. Ataev, Appl. Phys. Lett., 83, 2943, 2003

[102] Q.-X. Yu, B. Xu, Q.-H. Wu, Y. Liao, G.-Z. Wang, R.-C. Fang, H.-Y. Lee, and C.T. Lee, Appl. Phys. Lett. 83, 4713, 2003.

[103] Y. I. Alivov, J. E. Van Nostrand, D. C. Look, M. V. Chukichev, and B. M. Ataev, Appl. Phys. Lett., 83, 2943, 2003

[104] Q. X. Yu, B. Xu, Q.-H. Wu, Y. Liao, G. Z. Wang, R. C. Fang, H. Y. Lee, and C.T. Lee, Appl. Phys. Lett., 83, 4713, 2003

[105] M. C. Jeong, B. Y. Oh, M. H. Ham, and F. Myoung, Appl. Phys. Lett., 88, 202105, 2006

[106] O. Lupan, T. Pauporte, and B. Viana, Adv. Mater., 22, 3298, 2010

[107] Y. Chiba, A. Islam, Y. Watanabe, R. Komiya, N. Koide, and L. Han, Jpn. J. Appl. Phys., 45, 638, 2006

[108] R. S. Aga, D. Jowhar, A. Ueda, Z. Pan, W. E. Collins, R. Mu, K. D. Singer, and J. Shen, Appl. Phys. Lett., 91, 232108, 2007

[109] K. S. Leschkies, R. Divakar, J. Basu, E. Enache-Pommer, J. E. Boercker, C. B. Carter, U. R. Kortshagen, D. J. Norris, and E. S. Aydil, Nano Lett., 7, 1793, 2007 
[110] Y. Wu, C. Wadia, W. L. Ma, B. Sadtler, and P. A. Alivisatos, Nano Lett., 8, 2551, 2008

[111] E. H. Sargent, Nat. Photonics, 3, 325, 2009

[112] P. Hoyer and R. Könenkamp, Appl. Phys. Lett., 66, 349, 1995

[113] N. Zhao, T. P. Osedach, L. Chang, S. M. Geyer, D. Wanger, M. T. Binda, A. C. Arango, M. G. Bawendi, and V. Bulovic, ACS Nano 4, 3743, 2010

[114] M. G. Panthani, V. Akhavan, B. Goodfellow, J. P. Schmidtke, L. Dunn, A. Dodabalapur, P. F. Barbara, and B. A. Korgel, J. Am. Chem. Soc., 130, 16770, 2008

[115] K. T. Kuo, D. M. Liu, S. Y. Chen, and C. C. Lin, J. Mater. Chem., 19, 6780, 2009

[116] K. W. Johnston, A. G. Pattanthyus-Abraham, J. P. Clifford, S. H. Myrskog, D. D. MacNeil, L. Levina, and E. H. Sargent, Appl. Phys. Lett., 92, 151115, 2008

[117] G. I. Koleilat, L. Levina, H. Shukla, S. H. Myrskog, S. Hinds, A. G. PattantyusAbraham, and E.H. Sargent, ACS Nano 2, 833, 2008

[118] A. G. Pattantyus-Abraham, I. J. Kramer, A. R. Barkhouse, X. Wang, G.

Konstantatos, R. Debnath, L. Levina, I. Raabe, M. K. Nazeeruddin, M. Grätzel, and E. H. Sargent, ACS Nano 4, 3374, 2010

[119] K. Ernst, A. Belaidi, and R. Könenkamp, Semicond. Sci. Technol., 18, 475, 2003

[120] Y. Feng, D. Yun, X. Zhang, and W. Feng, Appl. Phys. Lett., 96, 093301, 2010

[121] Y. Zhou, F. S. Riehle, Y. Yuan, H.-F. Schleiermacher, M. Niggemann, G. A. Urban, and M. Kruger, Appl. Phys. Lett., 96, 013304, 2010

[122] S. Dayal, M. O. Reese, A. J. Ferguson, D. S. Ginley, G. Rumbles, N. Kopidakis, Adv. Funct. Mater., 20, 2629, 2010

[123] J. A. Chang, J. H. Rhee, S. H. Im, Y. H. Lee, H. J. Kim, S. Seok II, M. K. Nazeeruddin, M. Grätzel, Nano Lett., 10, 2609, 2010

[124] K. S. Leschkies, R. Divakar, J. Basu, E. Enache-Pommer, J. E. Boercker, C. B. Carter, U. R. Kortshagen, D. J. Norris, and E. S. Aydil, Nano Lett., 7, 1793, 2007

[125] Y. L. Lee, B.-M. Huang, and H.-T. Chien, Chem. Mater., 20, 6903, 2008

[126] A. Kongkanand, K. Tvrdy, K. Takechi, M. Kuno, and P. V. Kamat, 2008. J. Am. Chem. Soc., 130, 4007, 2008 
[127] S. Sukhovatkin, S. Hinds, L. Brzozowski, and E. H. Sargent, Science, 324, 1542, 2009

[128] J. J. H. Pijpers, R. Ulbricht, K. J. Tielrooij, A. Osherov, Y. Golan, C. Delerue, G. Allan, and M. Bonn, Nat. Phys. 5, 811, 2009

[129] J. Murphy, M. Beard, A. Norman, S. Ahrenkiel, J. Johnson, P. Yu, O. Mićić, R. Ellingson, A. Nozik, J. Am. Chem. Soc., 128, 3241, 2006

[130] R. Schaller, M. Sykora, S. Jeong, V. Klimov, J. Phys. Chem. B 110, 25332, 2006

[131] M. Beard, K. Knutsen, P. Yu, J. Luther, Q. Song, W. Metzger, R. Ellingson, and A. Nozik, Nano Lett., 7, 2506, 2007

[132] W. Shockley and H. J. Queisser, J. Appl. Phys., 32, 510, 1961

[133] R. Vogel, K. Pohl, H. Weller, Chem. Phys. Lett., 174, 241, 1990

[134] A. Mishra, M. K. R. Fischer, P. Bäuerle, Angew. Chem. Int. Ed., 48, 2474, 2009

[135] B. O’Regan and M. Grätzel, Nature, 353, 737, 1991

[136] T. Toyoda, T. Uehata, R. Suganuma, S. Tamura, A. Sato, K. Yamamoto, N. Kobayashi, Q. Shen, Jpn. J. Appl. Phys., 46, 4616, 2007

[137] Y.-L. Lee, C.-F. Chi, and S.-Y. Liau, Chem. Mater., 22, 922, 2010

[138] T. Dittrich, A. Belaidi, and A. Ennaoui, Sol. Energy Mater. Sol. Cells, 95, 1527, 2011

[139] J. B. Sambur, S. C. Riha, D. Choi, B. A. Parkinson, Langmuir, 26, 4839, 2010

[140] L. L. M. Peter, D. J. Riley, E. J. Tull, K. G. U. Wijayantha, Chem. Commun., 1030,2002

[141] L. J. Diguna, Q. Shen, J. Kobayashi, T. Toyoda, Appl. Phys. Lett., 91, 023116, 2007

[142] S. Chen, M. Paulose, C. Ruan, G. K. Mor, O. K. Varghese, D. Kouzoudis, C. A. Grimes, J. Photochem. Photobiol. A 177, 177, 2006

[143] A. Zaban, O. I. Mícíc, B. A. Gregg, A. J. Nozik, Langmuir 14, 3153, 1998 
[144] J. Chen, D. W. Zhao, J. L. Song, X. W. Sun, W. Q. Deng, X. W. Liu, W. Lei, Electrochem. Commun., 11, 2265, 2009

[145] K. Ernst, R. Engelhardt, K. Ellmer, C. Kelch, H. J. Muffler, M. C. Lux-Steiner, R. Könenkamp, Thin Solid Films, 387, 26, 2001

[146] K. Ernst, A. Belaidi, and R. Könenkamp, Sci. Technol., 18, 475, 2003

[147] R. Könenkamp, “Inorganic-extended junction devices: Nanostructured and Photoelectrochemical Systems for Solar Photon Conversion" Imperial College Press, pp. 393-452 (Chapter 6), 2008

[148] R. Könenkamp, L. Dloczik, K. Ernst and C. Olecsh, Physica, E 14, 219, 2002

[149] R. Tena-Zaera, M. A. Ryan, A. Katty, G. Hodes, S. Bastide and C. LévyClément, C. R. Chim., 9, 717, 2006

[150] G. Liu, T. Schulmeyer, A. Thissen, A. Klein, W. Jaegermann, Appl. Phys. Lett., 82, 2269, 2003.

[151] I. Kaiser, K. Ernst, C. Fischer, R. Könenkamp, C. Rost, I. Sieber, M. C. LuxSteiner, Solar Energy Materials and Solar Cells, 67, 89, 2001

[152] S. Siebentritt, K. Ernst, Ch.-H. Fischer, R. Könenkamp, M. C. Lux-Steiner, Proceedings of the $14^{\text {th }}$ European Photovoltaic Solar Energy Conference and Exibition, Barcelona, 1823, 1997

[153] C. Lévy-Clément, R. Tena-Zaera, M. A. Ryan, A. Katty, G. Hodes, Adv Mater., $17,1512,2005$

[154] C. Lévy-Clément, Nanostructured ETA-solar cells. In: T. Soga, editor, Nanostructured materials for solar energy conversion, Amsterdam: Elsevier, 2006

[155] F. Zenia, C. Levy-Clement, R. Triboulet, R. Konenkamp, K. Ernst, M. Saad, M. C. Lux-Steiner, Appl Phys Lett., 75, 531, 1999

[156] C. Lévy-Clément, R. Tena-Zaera, M. A. Ryan, A. Katty, and G. Hodes, Adv. Mater., 17, 1512, 2005

[157] H. Kawazoe, M. Yasukawa, H. Hyodu, M. Kurita, H. Yanagi, and H. Hosono, Nature, 389, 939, 1997

[158] A. Belaidi, T. Dittrich, D. Kieven, J. Tornow, K. Schwarzburg, M. Lux-Steiner, Phys. Stat. Sol. (RRL), 2, 172, 2008 
[159] Y. Itzhaik, O. Niitsoo, M. Page, G. Hodes, J. Phys. Chem C 113, 4254, 2009

[160] K. Taretto and U. Rau, Prog Photovoltaics Res Appl., 12, 573, 2004

[161] J. A. Chang, J. H. Rhee, S. H. Im, Y.H Lee, H.-J. Kim, S. Seok II, M. K. Nazeeruddin, and M. Grätzel, Nano Lett., 10, 2609, 2010

[162] R. Kniprath, J. P. Rabe, J. T. McLeskey, D. Wang, S. Kirstein, Thin Solid Films 518, 295, 2009

[163] K. S. Leschkies, T. J. Beatty, M. S. Kang, D. J. Norris, E. S. Aydil, ACS Nano $2009,3,3638-3648$.

[164] K. S. Leschkies, R. Divakar, J. Basu, E. E-Pommer, J.E. Boercker, C. B. Carter, U.R. Kortshagen, D.J. Norris and E.S. Aydil, Nano. Lett., 7, 1793, 2007

[165] W. U. Huynh, J.J. Dittmer, W.C. Libby, G.L. Whiting, A.P. Alivisatos, Adv. Funct. Mater., 13, 73, 2003

[166] S. Karuppuchamy, K. Nonomura, T. Yoshida, T. Sugiura, H. Minoura, Solid State Ionics, 151, 19, 2002

[167] Z. Wang, P. Xiao, J.J. Schemilt, Eur Ceramic Soc, 20, 1469, 2000

[168] Z. Wang, P. Xiao, J. J. Schemilt, Eur Ceramic Soc, 20, 1469, 2000

[169] B. O'Regan, D. T. Schwartz, S. M. Zakeeruddin, and M. Grätzel, Adv. Mater., 12, 1263,2000

[170] W. I. Park, G. C. Yi, J. W. Kim, S. M. Park, Appl. Phys. Lett. 82, 4358, 2003

[171] E. Boatman, G. Lisensky, and K. Nordell, J. Chem. Edu., 82, 2005

[172] B. J. Landia, C. M. Evansa, J. J. Wormana, S. L. Castrob, S. G. Baileyc. and R. P. Raffaelle, Mater. Lett., 60, 3502, 2006

[173] K. S. Leschkies, R. Divakar, J. Basu, E. Enache-Pommer, J. E. Boercker, C. Barry Carter, Uwe R. Kortshagen, D. J. Norris, and E. S. Aydil. Nano Lett., 7 (6), 1793, 2007

[174] A. Nadarajah, R. C. Word, and R. Könenkamp, Mater. Res. Soc. Symp. Proc., 1178,2009

[175] J. M. Luther, M. Law, Q. Song, C. L. Perkins, M. C. Beard, and A. Nozik, J. ACS Nano, 22, 271, 2008 
[176] S. A. Ringel, A. W. Smith, M. H. Macdougal, and A. Rohatgi, J. Appl. Phys., 70, 881,1991

[177] A. J. Breeze, Z. Schlesinger, S. A. Carter, and P. J. Brock, Phys. Rev. B, 64, 1252051,2001

[178] H. Kang, K. Kim, M. Kim, K. Park, K. Kim, T. Lee, J. Joo, K. Kmi, D. Lee, and J. Jin, Curr. Appl Phys. 1, 443, 2001

[179] A. R. Inigo, H. C. Chiu, W. Fann, Y.-S. Huang, U.-Ser Jeng, T.-L. Lin, C.-H. Hsu, K.-Y. Peng, and S.-A. Chen, Phys. Rev. B 69, 075201, 2004

[180] A. Kumar, P. K. Bhatnagar, P. C. Mathur, M. Husain, S. Sengupta And J. Kumar, J. Appl. Phys. 98, 024502, 2005

[181] L. Bozano, S. A. Carter, J. C. Scott, G. G. Malliaras, and P. J. Brock, Appl. Phys. Lett., 74, 1132, 1999

[182] A. C. Arango, S. A. Carter, and P. J. Brock, Appl. Phys. Lett., 74, 1698, 1999

[183] M. M. Alam and S. A. Jenekhe, Chem. Mater., 16, 4647, 2004

[184] K. Takahashi, K. Seto, T. Yamaguchi, J. Nakamura, C. Yokoe, and K. Murata, Chemistry Letters, 33, 8, 1042, 2004

[185] A. L. Holt, J. M. Leger, and S. A. Carter, J. Chem. Phys., 123, 044704, 2005

[186] S. A. Choulis, Y. Kim, J. Nelson, D. C. Bradley, M. Giles, and M. Shkunov, Appl Phys Lett., 85, 3890, 2004

[187] H. Sirringhaus, P. J. Brown, R. H. Friend, M. M. Nielsen, M. M, K. Bechgaard, B. M. W. Langeveld-Voss, A. J. H. Spiering, R. A. J. Janssen, E. W. Meijer, P. Herwig, and D. M. De Leeuw, Nature 401, 685, 1999

[188] T. Xu and Q. Qiao, Energy Environ. Sci., 4, 2700, 2011

[189] R. Engelhardt and R. Könenkamp, J. Appl. Phys., 90, 4287, 2001

[190] G. R. R. A. Kumara, A. Konno, G. K. R. Senadeera, P. V. V. Jayaweera, D. B. R. A. De Silva, K. Tennakone, Sol. Energy Mater. Sol. Cells, 69, 195, 2001

[191] T. S. Herng, S. P. Lau, S. F. YU, J. S. Chen, and K. S. Teng, Journal of Magnetism and Magnetic Materials., 315, 107, 2007 
[192] A. Hangleiter, D. Fuhrmann, M. Grewe, F. Hitzel, G. Klewer, S. Lahmann, C. Netzel, N. Riedel, and U. Rossow, Phys. Status Solidi A, 201, 2808, 2004

[193] S. W. Kim, S. Fujita, and S. Fujita, Appl. Phys. Lett., 86, 153119, 2005

[194] J. Chen, L. Aé, Ch. Aichele and M. Ch. Lux Steiner, Appl. Phys. Lett., 92, 161906, 2008

[195] A. Nadarajah and R. Könenkamp, Nanotechnology, 22, 025205, 2011

[196] J. C. Johnson, H. Yan, P. Yang, and R. J. Saykally, J. Phys. Chem. B, 107, 8816, 2003

[197] T. Dittrich, A. Belaidi, and A. Ennaoui, Sol. Energy Mater. Sol. Cells, 95, 1527, 2011

[198] A. J. Nozik, Chem. Phys. Lett., 457, 3, 2008

[199] W. A. Tisdale, K. J. Williams, B. A. Timp, D. J. Norris, E. S. Aydil, and X.-Y. Zhu, Science, 18, 1543, 2010

[200] B. Ehrler, M. W. B. Wilson, A. Rao, R. H. Friend, and N. C. Greenham, Nano Lett., 12 (2), 1053, 2012

[201] K. Ernst, R. Engelhardt, K. Ellmer, C. Kelch, H. J. Muffler, M. C. Lux-Steiner, R. Könenkamp, Thin Solid Films, 387, 26, 2001

[202] R. Könenkamp, A. Nadarajah, and R.C. Word, Appl. Phys. Lett., 95, 053103, 2009

[203] D. H. Kim, Y. D. Park, Y. S. Jang, H. C. Yang, Y. H. Kim, J. I. Han, D. G. Moon, S. J. Park, T. Y. Chang, C. W. Chang, M. K. Joo, C. Y. Ryu, and K. W. Cho, Adv. Func. Mater., 15, 77, 2005

[204] C. Levy-Clement, R. Tena-Zaera, M. A. Ryan, A. Katty, and G. Hodes, Adv. Mater., 17, 1512, 2005

[205] J. Rostalski, D. Meissner, Sol. Energy Mater. Sol. Cells, 61, 87, 2000

[206] J. M. Luther, M. Law, Q. Song, C. L. Perkins, M. C. Beard, A. J. Nozik, ACS Nano, 2, 271, 2008.

[207] E. J. D. Klem, D. D. MacNeil, P. W. Cyr, L. Levina, and E. H. Sargent, Appl. Phys. Lett., 90, 183113, 2007. 
[208] K. S. Leschkies, T. J. Beatty, M. S. Kang, D. J. Norris, E. S. Aydil, ACS Nano, 3, 3638,2009

[209] H. J. Morales-Rodríguez and F. Espinosa-Magaña, Micron, 48, 177, 2012 